\title{
Definable groups in Models of Presburger Arithmetic and $G^{00}$
}

\author{
Mariana Vicaría \\ Advisor: Alf Onshuus
}

September 23, 2016 


\section{Contents}

1 Preliminaries $\quad 9$

1.1 Some basic notions in Model theory . . . . . . . . . . . . . . . . . 11

1.1.1 Types and saturated models . . . . . . . . . . . . . 11

1.1.2 Dependent theories and indiscernible sequences . . . . . . . . 14

1.2 Groups and measures in dependent theories $\ldots \ldots \ldots \ldots$

1.2.1 Measures and Keisler Measures _. . . . . . . . . . . . . . . 19

1.2.2 Keisler measures in dependent theories . . . . . . . . . . . . 24

1.2 .3 Groups with $\mathrm{fsg} \ldots \ldots \ldots \ldots \ldots \ldots \ldots \ldots$

1.3 Amenable Groups . . . . . . . . . . . . . . . . . . . . . . . . 33

2 Analysis of definable groups in Presburger Arithmetic 45

2.1 Presburger Language and quantifier elimination . . . . . . . . . . . . 46

2.1.1 Some consequences of quantifier elimination $\ldots \ldots \ldots . \ldots 47$

2.1.2 A-definable functions and $\operatorname{dcl}(A) \ldots \ldots \ldots \ldots \ldots$

2.2 Cell Descomposition theorem . . . . . . . . . . . . . . . . 54

2.3 Dimension . . . . . . . . . . . . . . . . . . . . . 61

2.3.1 Definable Dimension . . . . . . . . . . . . . . . . . 61

2.3 .2 Cell Dimension . . . . . . . . . . . . . . . . . . . . . 64

2.3 .3 Open cells and boxes $\ldots \ldots \ldots \ldots \ldots$

3 A characterization of definable groups in Presburger Arithmetic $\quad 77$ 
3.1 Group operation vs usual addition . . . . . . . . . . . . . . . 77

3.2 Every definable group in Presburger is abelian-by-finite . . . . . . . . . . 84

3.2.1 The centralizer connected component and the ICC property . . . . 84

3.2.2 $G$ is abelian-by-finite . . . . . . . . . . . . . . . 87

4 Generic sets of definable groups in Presburger $\quad 91$

4.1 Definable functions between two non- algebraic types . . . . . . . . . . . 92

4.2 The theorem of the finite intersection property and a point . . . . . . . 95

4.3 Generic sets of definable bounded groups . . . . . . . . . . . . 107

5 Ellis semigroup conjecture $\quad 115$

5.1 Topological dynamics . . . . . . . . . . . . . . . . . . . . 115

5.2 The Ellis Group . . . . . . . . . . . . . . . . . . . . 117

5.3 Topological dynamics from a model theoretic approach . . . . . . . . . 125 


\section{Introduction}

In [7] (2008) Hrushovski, Peterzil and Pillay proved the following theorem:

Theorem 0.0.1. If $G$ is a definably compact group definable in a saturated o-minimal expansion of a real closed field, then the quotient $G / G^{00}$ of $G$ by its smallest type-definable subgroup of bounded index $G^{00}$, is, when equipped with the logic topology, a compact Lie group whose dimension (as a Lie group) equals the dimension of $G$ (as a definable set in an o-minimal structure).

In that paper these authors pointed out the connection between several topics, in order to classify the quotient group $G / G^{00}$. The knowledge areas involved in their paper are amenability in dependent theories, the finitely satisfiable generics property (fsg), the generic sets and the existence of compact group $H$ which dominates $G$, among others. Therefore, a natural question that might arise is: Can we generalize some of their results to other similar theories? How much all these theorems depend heavily on being in the o-minimal context?

In particular, we are interested in classifying the quotient group $G / G^{00}$, where $G$ is a definable group in the theory $T=T h(\mathbb{Z},+,<)$, which is known as the Presburger Arithmetic theory. Actually, this theory has some properties which are very close to the o-minimal case. Indeed, it is a dependent theory and moreover it is quasi o-minimal. Additionally, R. Cluckers in [3] proved that there is a cell decomposition theorem for this theory, which allows us to define also a notion of dimension for the definable sets, such as in the ominimal context.

However, we lose the topology induced on $G$ by the order $\leq$, since in the Presburger case it corresponds to the discrete topology. This is a huge loss, indeed the topological tools, such as continuity and the connected components, were fundamental in the o-minimal context to show that $G / G^{00}$ is a Lie group (see [14]). 
Nevertheless, in this thesis we show that we can recover some of the results obtained in the o-minimal theories for the Presburger case. For example, following some ideas of P. Eleftheriou and S. Starchenko in [13] and using the cell decomposition theorem we can show that every definable group in Presburger is abelian-by-finite, which implies that it is also definably amenable.

Additionally, Peterzil and Pillay have shown that the definable non-generic subsets of a definably compact abelian group $G$, in an o-minimal theory, are an ideal. The crucial idea of this proof is presented in Theorem 2.1 of [8], and there is a similar statement of this theorem that holds for the Presburger case, actually we realized that the condition of being definably compact might be changed by being bounded. Moreover, this will be enough to show that any definable bounded group in Presburger has the fsg property, which implies that the Ellis group conjecture of Newelski hold for the definable bounded groups in Presburger.

In the first chapter we introduce all the necessary concepts, tools and theorems that are going to be relevant for this work. More precisely, we present a detailed explanation of amenable groups, a summary of dependent theories and their relationship with Keisler measures, an exposition of the fsg property and how it implies a characterization of $G^{00}$ as a stabilizer. In the second chapter, we introduce the cell decomposition theorem developed by R. Cluckers and we present two notions of dimension for the definable sets in the Presburger theory, one comes from a definable independence, while the second one arises from the cell decomposition theorem. Actually, we show that this two notions coincide. In the third chapter, we show that every definable group in Presburger is abelian-by-finite, while in Chapter 4 we present a similar statement of the Theorem 2.1 of [8], in order to show that every definable bounded group in Presburger has the fsg property. In Chapter 
5, we present a proof developed by A. Pillay in [9] which establishes that the Ellis group conjecture of Newelski holds for every definable group with the fsg property.

\section{Acknowledgements}

Entregar esta tesis implica que me despido de los Andes, por lo que mis agradecimientos no los dirigiré únicamente a cosas relativas a este trabajo. Esta tesis es simplemente el último paso, de muchos. Sin embargo, empezaré por agradecerle a Alex y a Juan Felipe por sus útiles correcciones y detallada lectura.

Le debo a los Andes seis años de mucha mucha felicidad, y sí, claro que tengo nostalgia. Me despido de las tardes en el sótano haciendo las interminables tareas de Monika o de Ramiro, me despido del café del departamento y de los cigarrillos compartidos al frente del H. Me despido de las guaridas secretas y los abrazos sigilosos. Me despido del fútbol, de la copa Klein, de los goles tambaleantes y flojos. Le digo adiós a los miércoles de cerveza en el BBC y al bullying del grupo de lógica. Me despido de mi segunda casa. Me despido de profesores que admiro con toda la integridad del caso, por su dedicación en sus clases y por hacer de mi aprendizaje algo siempre mejor. Gracias a Carlos, Monika y a Xavier por dejarme ver el gran cariño que sienten por lo que hacen y, en particular, por dejar algo de esa dedicación en mi.

Me despido de mi asesor, y sobretodo mi amigo, Alf, a quien le estaré eternamente agradecida por su incondicional apoyo y su confianza. Gracias por los cafés, cigarrillos, goles, charlas, genialidades y falsedades de las que nos convencimos. Gracias por haber creído más en mí de lo que yo creo. Gracias por la paciencia, y por mostrarme que las matemáticas, más que mi deber, son algo que disfruto.

También me despido de mis amigos, de las canciones pussy de Mateín, de los chistes os-

curos de Lucho, del idealismo fascinante de Andy y del persistente color negro de Monika. 
Me despido del inolvidable Sena de Samy, de las cervezas de Rafa, de la ligereza de Santi y la franqueza de Juan Felipe. Me despido de las tardes de billar con Dani, del gordelio de Alemanchis y de la versión hippie de Calderón.

Me voy con lágrimas en los ojos motivadas por una inmensa alegría de haberlos conocido, y anhelando que la vida eventualmente vuelva a ponernos en Magola. A muchos es posible que no los vuelva a ver, así que: buen viento y buena mar.

Por supuesto, debo mencionar también el profundo agradecimiento que siento con mi familia por su constante apoyo. En particular, debo agradecerle a mis papás por cada uno de los esfuerzos que hicieron a lo largo de su vida para darme la oportunidad de haber estado en los Andes. Les agradezco también haber visto mucho más allá de las expectativas que tenían para mí, y haberse preocupado sobretodo por mi felicidad. He de decirles, con toda la seguridad que puedo atribuirme, que fui extremadamente feliz. Gracias a ustedes ésto fue posible. 


\section{Chapter 1}

\section{Preliminaries}

\section{Notational convention}

The main purpose of this section is to introduce all the notation that will be used throughout this thesis.

- Given sets $X, Y$, we sometimes denote the set of all functions $f: X \rightarrow Y$ by $Y^{X}$.

- Given a complete theory $T, \mathcal{M}$ will denote a saturated model and $\mathcal{M}_{0}$ will be a small elementary submodel of $\mathcal{M}$.

- $G$ will always denote a definable group in a complete theory $T$. Particularly, during Chapters 2 and 3, $G$ will denote a definable group in the theory of Presburger Arithmetic.

- The notation $\bar{a}$ will denote a tuple of elements of $\mathcal{M}$, and given a tuple $\bar{a} \in \mathcal{M}^{n}$, say $\bar{a}=\left(a_{1}, \ldots, a_{n}\right)$ we use $(\bar{a})_{i}$ to denote the $i$-th coordinate of $\bar{a}$, which is $a_{i}$.

- Let $X \subseteq \mathcal{M}^{\ell}$ and let $0 \leq n<\ell$, we indicate by $\pi_{\leq n}(X)$ the projection of $X$ on the first $n$ coordinates, that is,

$$
\pi_{\leq n}(X):=\left\{\left(x_{1}, \ldots, x_{n}\right) \mid\left(x_{1}, \ldots, x_{n}, x_{n+1}, \ldots, x_{l}\right) \in X\right\}
$$


- In the structures that we are going to study throughout this thesis we consider a linear order $<$ that belongs to the language of Presburger Arithmetic. Thus, we introduce some standard notation for the intervals as follows:

- we indicate by $[a, b]$ the set defined as $\{x \in \mathcal{M} \mid a \leq x \leq b\}$,

- we denote by $(a, b)$ the set defined as $\{x \in \mathcal{M} \mid a<x<b\}$.

- If $X$ and $Y$ are subsets of $\mathcal{M}$ we say that $X<Y$ if and only if for every pair of elements $x \in X$ and $y \in Y$ we have that $x<y$

- If $G$ is a group and $H$ is a subgroup, the cardinal $[G: H]$ is called the index on $H$ in $G$ and denotes the number of left cosets of $H$ in $G$.

- A system of linear congruences will be a finite set of equations of the form $x \equiv_{N}$ $c$, where $0 \leq c<N$ are integers. Throughout this thesis, we will be using the generalization of the chinese reminder theorem, which says that a consistent linear system of congruences

$$
x \equiv_{N_{1}} c_{1}, \ldots, x \equiv_{N_{k}} c_{k}
$$

can be reduced to a unique equation $x \equiv_{N} c$.

- Another result that we are going to recall several times is theErdos- Rado Theorem, which says:

Let $\kappa$ be a cardinal and $r, n \in \mathbb{N}$. For every partition of $[\kappa]^{n}=\{A \subseteq \kappa:|A|=n\}$ into $r$ pieces; then there is a subset $\lambda \leq \kappa$ such that $[\lambda]^{n}$ is completely contained in exactly one of the classes. 


\subsection{Some basic notions in Model theory}

\subsubsection{Types and saturated models}

The main purpose of the first part of this section is to introduce several definitions that we are going to use through this thesis.

Definition 1.1.1 (An $n$-type over $A$ ). Fix a set of parameters $A$. An $n$-type over $A$ is a consistent set $\pi\left(x_{1}, \ldots, x_{n}\right)$ of $\mathcal{L}(A)$-formulas in $n$ variables, $x_{1}, \ldots, x_{n}$. We say that an $n$-type $p\left(x_{1}, \ldots, x_{n}\right)$ is complete if it is a maximal consistent set.

Given an $n$-tuple $\bar{b}=\left(b_{1}, \ldots, b_{n}\right)$ from a model $\mathcal{M}$ and a subset of parameters $A \subseteq \mathcal{M}$, we denote by $\operatorname{tp}(\bar{b} / A)$ the complete $n$-type over $A$ given by

$$
\operatorname{tp}(\bar{b} / A):=\left\{\phi\left(x_{1}, \ldots, x_{n} ; \bar{a}\right): \bar{a} \in A \text { and } \mathcal{M} \models \phi\left(b_{1}, \ldots, b_{n} ; \bar{a}\right)\right\}
$$

Definition 1.1.2 (global type). Let $\mathcal{M}$ be a very saturated model of a theory $T$. A global type is simply a complete $n$-type $p\left(x_{1}, \ldots, x_{n}\right)$ over $\mathcal{M}$.

Definition 1.1.3 (saturated model). Let $T$ be a theory and $\mathcal{M}$ a model of $T$. We say that $\mathcal{M}$ is $\kappa$-saturated if for every set of parameters $A$, such that $|A|<\kappa$, and any $n$-type $\pi\left(x_{1}, \ldots, x_{n}\right)$ over $A$, we can find $b_{1}, \ldots, b_{n} \in \mathcal{M}$ such that:

$$
\mathcal{M} \models \pi\left(b_{1}, \ldots, b_{n}\right)
$$

If $\kappa=|\mathcal{M}|$ and $\mathcal{M}$ is $\kappa$-saturated, then we say that $\mathcal{M}$ is saturated.

Definition 1.1.4 (small set of parameters). If $\mathcal{M}$ is a $\kappa$-saturated model, we say that $A$ is a small set of parameters if $|A|<\kappa$. An elementary submodel $\mathcal{M}_{0}$, is a small submodel of $\mathcal{M}$ if $\left|\mathcal{M}_{0}\right|<\kappa$.

Definition $\mathbf{1 . 1 . 5}$ (finitely satisfiable type over $\mathcal{M}_{0}$ ). Let $\mathcal{M}_{0}$ be a small submodel of $\mathcal{M}$. Let $\pi\left(x_{1}, \ldots, x_{n}\right)$ be an $n$-type over a set of small set of parameters $A$. We say that 
$\pi\left(x_{1}, \ldots, x_{n}\right)$ is finitely satisfiable over $\mathcal{M}_{0}$ if for every finite subset $\pi_{0}=\left\{\phi_{i}\left(x_{1}, \ldots, x_{n}, \bar{a}\right)\right.$ : $i \leq k\} \subseteq \pi\left(x_{1}, \ldots, x_{n}\right)$, there is a tuple $\left(b_{1}, \ldots, b_{n}\right) \in \mathcal{M}_{0}$ such that $\mathcal{M}_{0} \models \pi_{0}$, that is, $\mathcal{M}_{0} \models \phi_{1}\left(b_{1}, \ldots, b_{n}, \bar{a}\right) \wedge \cdots \wedge \phi_{k}\left(b_{1}, \ldots, b_{n}, \bar{a}\right)$.

Example 1.1.6. - Let $T$ be the theory of the dense linear orders and let $\mathcal{M}_{0}=(\mathbb{Q},<)$. Consider the type $\pi(x)=\{x>n \mid n \in \mathbb{N}\}$, then $p(x)$ is finitely satisfiable over $\mathcal{M}_{0}$ : Note that for every $n_{1}<\cdots<n_{k}$ in $\mathbb{N}$ we have that $n_{k}+1 \in \mathcal{M}_{0}$ satisfies $n_{k}+1>n_{1} \wedge n_{k}+1>n_{2} \wedge \cdots \wedge n_{k}+1>n_{k}$.

- Let $T$ be the theory of the fields of characteristic 0 and let $p(x)=\operatorname{tp}(i / \mathbb{R})$. This type is not finitely satisfiable over $\mathbb{R}$, since the formula $x^{2}+1=0 \in p(x)$, but we know that there is no solution for the equation $x^{2}+1=0$ in the field of real numbers.

Definition 1.1.7 (Invariant type). Let $\mathcal{M}_{0}$ be a small model such that $\mathcal{M}_{0} \prec \mathcal{M}$, where $\mathcal{M}$ is a saturated model over $\mathcal{M}_{0}$. Let $p(\bar{x})$ be a complete type over $\mathcal{M}$ we say that it is $\mathcal{M}_{0}$-invariant if for any formula $\phi(\bar{x}, b)$ and element $b \in \mathcal{M}$, wheter or not $\phi(\bar{x}, b) \in p(\bar{x})$ depends only on the type $t p\left(b / \mathcal{M}_{0}\right)$. In other words:

if $t p\left(b / \mathcal{M}_{0}\right)=t p\left(b^{\prime} / \mathcal{M}_{0}\right)$, then $\phi(\bar{x}, b) \in p(\bar{x})$ if and only if $\phi\left(\bar{x}, b^{\prime}\right) \in p(\bar{x})$.

Remark 1.1.8. Let $\mathcal{M}_{0}$ be an elementary submodel of $\mathcal{M}$ and assume that $\mathcal{M}$ is saturated over $\mathcal{M}_{0}$, and let $p(\bar{x})$ be a complete type over $\mathcal{M}$. If $p(\bar{x})$ is finitely satisfiable over $\mathcal{M}_{0}$ then it is also $\mathcal{M}_{0}$-invariant.

Proof. Let $p(\bar{x})$ be a complete type over $\mathcal{M}$, which is finitely satisfiable over $\mathcal{M}_{0}$, and let $\phi(\bar{x}, \bar{y})$ be a formula. Let $\bar{b}, \bar{b}^{\prime}$ be two elements such that $\operatorname{tp}\left(\bar{b} / \mathcal{M}_{0}\right)=\operatorname{tp}\left(\bar{b}^{\prime} / \mathcal{M}_{0}\right)$. In order to find a contradiction, assume that $\phi(\bar{x}, \bar{b}) \in p(\bar{x})$ but $\neg\left(\phi\left(\bar{x}, \bar{b}^{\prime}\right)\right) \in p(\bar{x})$. By completeness $\phi(\bar{x}, \bar{b}) \wedge \neg\left(\phi\left(\bar{x}, \bar{b}^{\prime}\right)\right) \in p(x)$, and since $p(\bar{x})$ is finitely satisfiable there is an element $\bar{m}$ of $\mathcal{M}_{0}$ such that $\mathcal{M} \vDash \phi(\bar{m}, \bar{b}) \wedge \neg\left(\phi\left(\bar{m}, \bar{b}^{\prime}\right)\right)$. This implies that $\phi(\bar{m}, \bar{y}) \in \operatorname{tp}\left(\bar{b} / \mathcal{M}_{0}\right)$ and $\neg(\phi(\bar{m}, \bar{y})) \in \operatorname{tp}\left(\bar{b}^{\prime} / \mathcal{M}_{0}\right)$, then we conclude that $\operatorname{tp}\left(\bar{b} / \mathcal{M}_{0}\right) \neq t p\left(\bar{b}^{\prime} / \mathcal{M}_{0}\right)$. 
Definition 1.1.9. Let $I=\left\langle\bar{a}_{i} \mid i \in \omega\right\rangle$ be a sequence of tuples in $\mathcal{M}$ and $B \subset \mathcal{M}$ a set of parameters. We say that $I$ is a $B$-indiscernible sequence if for every $\mathcal{L}(B)$-formula $\phi\left(\bar{x}_{1}, \cdots, \bar{x}_{n} ; \bar{b}\right)$ and $i_{1}<\cdots<i_{n}, j_{1}<j_{2}<\cdots j_{n} \in \omega$, we have that:

$$
\mathcal{M} \models \phi\left(\bar{a}_{i_{1}}, \cdots, \bar{a}_{i_{n}} ; \bar{b}\right) \leftrightarrow \mathcal{M} \models \phi\left(\bar{a}_{j_{1}}, \cdots, \bar{a}_{j_{n}} ; \bar{b}\right)
$$

Example 1.1.10. Let $T$ be the theory of dense linear orders without endpoints. Let $\mathcal{M}=(\mathbb{Q},<)$, then:

- let $p \in \mathbb{Q}$, the sequence defined as $a_{i}=p$ for all $i \in \omega$ is indiscernible.

- let $a_{0}<a_{1}<\ldots$ an increasing sequence of elements of $\mathbb{Q}$, by the quantifier elimination of $T, I=<a_{i} \mid i \in \omega>$ is indiscernible.

Definition 1.1.11 (EM-type). Let $I=\left\langle\bar{a}_{i} \mid i \in \alpha\right\rangle$ be a sequence, its EhrenfeuchtMostowski $(\mathrm{EM})$ type over a set of parameters $B$ is the set of the $\mathcal{L}(B)$-formulas that hold for every element $\bar{a}_{i} \in I$.

$$
E M(I / B)=\left\{\phi(\bar{x}, \bar{b}) \mid \text { for all } i \in \alpha \mathcal{M} \vDash \phi\left(\bar{a}_{i}, \bar{b}\right)\right\}
$$

Definition 1.1.12 (Dividing formula). Let $\phi(\bar{x} m \bar{a})$ be a formula and $A$ be a set of parameters. We say that $\phi(\bar{x}, \bar{a})$ divides over $A$ if there is an $A$-indiscernible sequence $<\bar{a}_{i} \mid i<\omega>\left(\right.$ where $\left.\bar{a}_{0}=\bar{a}\right)$ such that the set:

$$
\left\{\phi\left(\bar{x}, \bar{a}_{i}\right) \mid i<\omega\right\}
$$

is inconsistent.

Definition 1.1.13 (Forking). Let $\phi(\bar{x}, \bar{a})$ be a formula and $A$ be a set of parameters. We 
say that $\phi(\bar{x}, \bar{a})$ forks over $A$ if

$$
\phi(\bar{x}, \bar{a}) \longleftrightarrow \bigvee_{i=1}^{n} \psi\left(\bar{x}, \bar{b}_{i}\right)
$$

where each formula $\psi\left(\bar{x}, \bar{b}_{i}\right)$ divides over $A$.

Lemma 1.1.14. Let $I=\left\langle\bar{a}_{i} \mid i \in \omega\right\rangle$ be a sequence, then we can find an indiscernible sequence $C=\left\langle\bar{c}_{i} \mid i \in \omega\right\rangle$ such that $C$ satisfies the EM-type of $I$.

Proof. For a detailed explanation we refer the reader to [15] Lemma 5.13.

Definition 1.1.15 (Bounded Relation). Let $X$ be a definable set. Assume that $\sim$ is an equivalence relation defined over $X$. We say that $\sim$ has boundedly many classes if $|X / \sim|<|\mathcal{M}|$, where $\mathcal{M}$ is at least a $\left.2^{|T|}\right|_{\text {-saturated model. In this case, we also say that }}$ $\sim$ has boundedly many classes.

\subsubsection{Dependent theories and indiscernible sequences}

The main purpose of this section is to introduce some relevant facts about dependent theories. For a more detailed exposition see [10].

Definition 1.1.16 (Independence property and dependent formulas). Let $\phi(\bar{x}, \bar{y})$ be an $\mathcal{L}$ - formula, where $\bar{x}$ is a $k$-tuple.

1. We say that $\phi(\bar{x}, \bar{y})$ has the independence property if for any $n \in \omega$, there are elements $\overline{a_{1}}, \ldots, \overline{a_{n}} \in \mathcal{M}^{k}$ such that for each subset $S \subseteq\{1, \cdots, n\}$, there is an element $\bar{b}_{S} \in \mathcal{M}^{l}$, such that

$$
\mathcal{M} \models \phi\left(\bar{a}_{i}, \bar{b}_{S}\right) \text { if and only if } i \in S \text {. }
$$

In this case we say that the set $A=\left\{\bar{a}_{1}, \ldots, \bar{a}_{n}\right\}$ is shattered by $\phi(\bar{x}, \bar{y})$. 
2. We say that $\phi(\bar{x}, \bar{y})$ is an dependent formula if it does not have the independence property.

3. An $\mathcal{L}$-theory $T$ is a dependent theory if any formula $\phi(\bar{x} ; \bar{y})$ is a dependent formula.

Example 1.1.17. $\quad$ Let $T$ be theory of dense linear orders and consider the formula $\phi(x, y)=x \leq y$. Observe that $\phi(x, y)$ is a dependent formula:

Let $\mathcal{M}$ be a saturated model of $T$, and fix two elements different $p, q \in \mathcal{M}$. Let $A=\{p, q\}$ and without loss of generality assume that $p<q$. Let $b \in \mathcal{M}$ be any element, then we have the following cases:

- if $b<p$ then $A \cap \phi(\mathcal{M}, b)=\emptyset$,

- if $p \leq b<q$ then $A \cap \phi(\mathcal{M}, b)=\{p\}$,

- if $q \leq b$ then $A \cap \phi(\mathcal{M}, b)=\{p, q\}$

Therefore, we can not find some $b \in \mathcal{M}$ such that $A \cap \phi(\mathcal{M}, b)=\{q\}$, hence this formula does not have the independence property.

- Let $T$ be the theory of Peano Arithmetic and consider the formula $\phi(x, y)=x \mid y$, which means " $x$ divides $y^{\prime \prime}$.Then $\phi(x, y)$ has the independence property:

Let $N \in \mathbb{N}$ and consider $A=\left\{p_{0}, \ldots, p_{N-1}\right\}$ the first $N$-primes. Let $S$ be a subset of $\{0, \ldots, N-1\}$, and let $b_{S}=\prod_{i \in S} p_{i}$, therefore:

$$
\mathcal{M} \models \phi\left(p_{i}, b_{s}\right) \text { if and only if } i \in S \text {. }
$$

Therefore, $\phi(x, y)$ is an independent formula.

Example 1.1.18. The following are examples of dependent theories:

- Any o-minimal theory is dependent. [see [10], Chapter 2]

- Any stable theory is dependent. [see [10], Section 2.3.2] 
- Any quasi o-minimal theory is dependent, in particular Presburger Arithmetic is a dependent theory. [see [2]]

Definition 1.1.19 (shattered set). Let $\mathcal{M}$ be a model of a theory $T$. Let $\phi(\bar{x}, \bar{y})$ be a formula, then we say that this formula shatters a subset $A$ of $\mathcal{M}^{|\bar{x}|}$ if for any subset $S \in \mathcal{P}(A)$, there is an element $\bar{b}_{S}$ such that:

$$
\mathcal{M} \vDash \phi\left(\bar{a}, \bar{b}_{S}\right) \leftrightarrow \bar{a} \in S
$$

Remark 1.1.20. Let $\phi(\bar{x}, \bar{y})$ be a formula, then it has the independe property if and only if for any $n<\omega$ there is some set $A$, which is shattered by $\phi(\bar{x}, \bar{y})$.

Remark 1.1.21. A formula $\phi(\bar{x} ; \bar{y})$ has the independence property if and only if the formula $\psi(\bar{y} ; \bar{x}):=\phi(\bar{x} ; \bar{y})$ has the independence property. That is, for every $n<\omega$ there are also elements $\bar{b}_{1}, \ldots, b_{n} \in \mathcal{M}^{|y|}$ such that for each $S \subseteq\{1,2, \ldots, n\}$ there is $\bar{a}_{S} \in \mathcal{M}^{|\bar{x}|}$ such that $\mathcal{M} \models \phi\left(\bar{a}_{S} ; \bar{b}_{i}\right)$ if and only if $i \in S$.

Theorem 1.1.22. Let $\phi(\bar{x}, \bar{y})$ be a formula. This formula has the independence property if and only if there is an indiscernible sequence $\left\langle\bar{a}_{i} \mid i \in \omega\right\rangle$ and a tuple $\bar{b} \in \mathcal{M}^{l}$ such that:

$$
\mathcal{M} \models \phi\left(\bar{a}_{i}, \bar{b}\right) \text { if and only if } i \text { is even. }
$$

Proof. $(\Leftarrow)$ Assume that there is a sequence $\left\langle\bar{a}_{i} \mid i \in \omega\right\rangle$ and a tuple $\bar{b} \in \mathcal{M}^{l}$ as above. Let $I \subseteq \omega$, we will show that there is some $\bar{b}_{I}$ such that $\mathcal{M} \models \phi\left(\bar{a}_{i}, \bar{b}_{I}\right)$ if and only if $i \in I$.

Claim: We can find an increasing one-to-one map $\tau: \omega \rightarrow \omega$ such that for all $i \in \omega$ $\tau(i)$ is even if and only if $i \in I$

We will build this function by induction. If $0 \in I$, then we define $\tau_{0}(0)=0$, otherwise we define $\tau_{0}(0)=1$. 
Suppose that we have constructed an injective map $\tau_{n}:\{1, \cdots, n\} \rightarrow \omega$, then $\operatorname{Im}\left(\tau_{n}\right)$ is bounded in $\omega$. If $n+1 \in I$, then let $m$ the minimal element in $\omega \backslash \operatorname{Im}\left(\tau_{n}\right)$ which is even, and define $\tau_{n+1}=\tau_{n} \cup\{(n+1, m)\}$. If $n+1 \notin I$, let $\tau_{n+1}=\tau_{n} \cup\{(n+1, m+1)\}$. By construction $\tau_{n+1}$ is injective and increasing.

Let $\tau=\bigcup_{n \in \omega} \tau_{n}$, then $\tau$ is injective, increasing and $\tau(i)$ is even if and only if $i \in I . \quad \square$ Claim

Now, since $\left\langle\bar{a}_{i} \mid i \in \omega\right\rangle$ is an indiscernible sequence and $\tau$ is increasing, the map sending each $\bar{a}_{i}$ to $\bar{a}_{\tau(i)}$ is a partial isomorphism, and we can extend this function to a global automorphism $\sigma$ of $\mathcal{M}$. Let $\bar{b}_{I} \in \mathcal{M}^{l}$ such that $\sigma\left(\bar{b}_{I}\right)=\bar{b}$.

Thus, $\mathcal{M} \models \phi\left(\bar{a}_{i}, \bar{b}_{I}\right)$ if and only if $\mathcal{M} \models \phi\left(\bar{a}_{\tau(i)}, \bar{b}\right)$ if and only if $\tau(i)$ is even if and only if $i \in I$. We conclude that $\phi(\bar{x}, \bar{y})$ has the independence property.

$(\Rightarrow)$ Assume that $\phi(\bar{x}, \bar{y})$ has the independence property, and let $A=\left\langle\bar{a}_{i} \mid i \in \omega\right\rangle$ be a sequence of $|\bar{x}|$-tuples which is shattered by $\phi(\bar{x}, \bar{y})$. By Ramsey and compactness (Lemma 1.1.14) we can find some indiscernible sequence $I=\left\langle\bar{c}_{i} \mid i \in \omega\right\rangle$ of $|\bar{x}|$ - tuples satisfying the $E M$ - type of $A$. Note that for any two disjoint finite sets $I_{0}$ and $I_{1}$ of $I$ the partial type $\left\{\phi(\bar{c}, \bar{y}) \mid \bar{c} \in I_{0}\right\} \cup\left\{\neg(\phi(\bar{c}, \bar{y})) \mid \bar{c} \in I_{1}\right\}$ is consistent. In particular the type $\Gamma(y)=\left\{\phi\left(\bar{a}_{2 i} ; \bar{b}\right) \wedge \neg \phi\left(\bar{a}_{2 i+1} ; \bar{b}\right): i<\omega\right\}$ is finitely consistent, and by compactness there is an element $\bar{b} \in \mathcal{M}^{l}$ realizing $\Gamma(y)$. So, $\mathcal{M} \models \phi\left(\bar{c}_{i} ; \bar{b}\right)$ if and only if $i$ is even.

Lemma 1.1.23. $T$ is a dependent theory if and only if for any indiscernible sequence $\left\langle\bar{a}_{i} \mid i \in \omega\right\rangle$, and every formula $\phi(\bar{x})$ (possibly with parameters) there is an $i \in \omega$ such that either $\mathcal{M} \models \phi\left(\bar{a}_{j}\right)$ for all $j>i$, or $\mathcal{M} \models \neg\left(\phi\left(\bar{a}_{j}\right)\right)$ for all $j>i$.

Proof. $(\Rightarrow)$ Assume that $T$ is a dependent theory and let $I=\left\langle\bar{a}_{i} \mid i \in \omega\right\rangle$ an indiscernible sequence and $\phi(\bar{x}, \bar{b})$ a formula and $\bar{b} \in \mathcal{M}^{l}$. If the conclusion does not hold, then for every $i<\omega$ there are $i \leq j_{0}<j_{1}$ such that $\mathcal{M} \models \phi\left(\bar{a}_{j_{0}} ; \bar{b}\right) \wedge \neg \phi\left(\bar{a}_{j_{1}} ; \bar{b}\right)$.

This allows us to consider a subsequence $S=\left\langle\bar{a}_{i_{j}}: j<\omega\right\rangle$ such that $\mathcal{M} \models \phi\left(\bar{a}_{i_{j}} ; \bar{b}\right)$ if and only if $j$ is even: to start, take $j_{0}<j_{1}$ minimal such that $\phi\left(\bar{a}_{j_{0}}, \bar{b}\right) \wedge \neg \phi\left(\bar{a}_{j_{1}}, \bar{b}\right)$ and 
put $\bar{a}_{i_{0}}=\bar{a}_{j_{0}}, \bar{a}_{i_{1}}=\bar{a}_{j_{1}}$. Now, apply the same for $i=i_{1}$ to find elements $\bar{a}_{i_{2}}, \bar{a}_{i_{3}}$ such that $\phi\left(\bar{a}_{i_{2}}, \bar{b}\right) \wedge \neg \phi\left(\bar{a}_{i_{3}}, \bar{b}\right)$. Continuing with this construction we obtain the desired sequence. Since $S$ is an increasing subsequence of $B$, it is also indiscernible. By Theorem 1.1.22, we conclude that $\phi(\bar{x}, \bar{y})$ has the independence property. Thus $T$ is not a dependent theory.

$(\Leftarrow)$ Assume that there is a formula $\phi(\bar{x}, \bar{y})$ that has the independence property. By Theorem 1.1.22, there is an indiscernible sequence $\left\langle a_{i} \mid i \in \omega\right\rangle$ and a tuple $\bar{b}$ such that $\mathcal{M} \models \phi\left(\bar{a}_{i}, \bar{b}\right)$ if and only if $i$ is even. Then, it is impossible to find an element $i \in \omega$, such that either $\mathcal{M} \models \phi\left(\bar{a}_{j}, \bar{b}\right)$ for all $j>i$, or $\mathcal{M} \models \neg\left(\phi\left(\bar{a}_{j}, \bar{b}\right)\right)$ for all $j>i$.

Remark 1.1.24. We can say that $\phi(\bar{x})$ has an average value in the sequence $\left\langle\bar{a}_{i}: i \in \omega\right\rangle$ if there is $i \in \omega$ such that $\mathcal{M} \models \phi\left(\bar{a}_{j}\right) \leftrightarrow \phi\left(\bar{a}_{j+1}\right)$ for all $j>i$, which is the conclusion of the previous lemma.

Thus, Lemma 1.1.23 can be stated as follows: $T$ is a dependent theory if and only if every formula $\phi(\bar{x}) \in \mathcal{L}(\mathcal{M})$ has average value in every indiscernible sequence.

Given an indiscernible sequence $I=\left\langle\bar{a}_{i}: i<\omega\right\rangle$ in a dependent theory, the set of formulas $\phi(\bar{x} ; \bar{b})$ that holds cofinally in $I$ is a complete type, known as the average type of the sequence $I$.

Corollary 1.1.25. Suppose that $T$ is a dependent theory. Let $\phi(\bar{x}, \bar{y})$ be an $\mathcal{L}$-formula and $\left\langle\bar{b}_{i} \mid i \in \omega\right\rangle$ an indiscernible sequence. Then, $\left\{\phi\left(\bar{x}, \bar{b}_{2 j}\right) \triangle \phi\left(\bar{x}, \bar{b}_{2 j+1}\right) \mid j \in \omega\right\}$ is inconsistent.

Proof. Assume by contradiction that it is consistent, since $\mathcal{M}$ is saturated, there is an element $\bar{c}$ such that for all $j \in \omega, \mathcal{M} \models \phi\left(\bar{c}, \bar{b}_{2 j}\right) \triangle \phi\left(\bar{c}, \bar{b}_{2 j+1}\right)$. This contradicts Lemma 1.1.23, since the formula $\phi(\bar{c} ; y)$ will not have average value in $\left\langle\bar{b}_{i} \mid i \in \omega\right\rangle$.

\subsection{Groups and measures in dependent theories}

In [7] Hrushovski, Peterzil and Pillay stated several ideas relating measures and the dependence property, in order to prove that any definably compact group in an o-minimal 
theory has the fsg property. However, in order to understand their statements we will need to explain several tools, such as measures, Keisler measures and Keisler invariant measures, which are explained in Section 1.2.1, based on the book [10] of P. Simon, who did a very detailed explanation of these topics on Chapter 7.

Secondly, we present a detailed exposition of the main propositions of [7], in particular we recall those results that establish a relationship between the existence of an invariant measure and some properties of the dependent theories. Since those statements are going to be relevant to show that any definable bounded group in Presburger Arithmetic theory have the fsg property, we explain all the theory developed by Hrushovski, Peterzil and Pillay in the Section 1.2.3.

Additionally, in Subsection 1.1.3 we define the fsg property for definable groups in dependent theories, and we recall some relevant statements developed in [7], in order to characterize $G^{00}$ as the stabilizer of a global generic type.

In the following statements we will be working with $T$ a complete dependent theory, and $\mathcal{M}$ will indicate a very saturated model of $T$.

\subsubsection{Measures and Keisler Measures}

Definition 1.2.1. Let $X$ be a set. A collection $\Omega$ of subsets of $X$ is called a boolean algebra on $X$ if $\emptyset, X \in \Omega$, and $\Omega$ is closed under complements and finite unions. If in addition $\Omega$ is closed under countable unions, we say that it is a $\sigma$-algebra.

Example 1.2.2. Let $X$ be a definable set. Then the following sets are boolean algebras on $X$ :

- the set $\mathcal{P}(X)$ is a boolean algebra on $X$. 
- the set $\operatorname{Def}_{A}(X)=\{Y \subseteq X \mid Y$ is $A$-definable $\}$. We denote simply as $\operatorname{Def}(X)$ the set of the definable subsets of $X$ in $\mathcal{M}$.

Definition 1.2.3 (Measures). Let $X$ be a set, and $\Omega$ be a boolean algebra on $X$.

1. A finitely additive measure on $X$ (with respect to $\Omega$ ) if a function $\mu: \Omega \rightarrow \mathbb{R}^{\geq 0} \cup\{\infty\}$ satisfying the following:

(a) $\mu(\emptyset)=0$

(b) If $A_{1}, \cdots, A_{n}$ are pairwise disjoint elements in $\Omega$ then

$$
\mu\left(\bigcup_{i=1}^{n} A_{i}\right)=\sum_{i=1}^{n} \mu\left(A_{i}\right)
$$

2. If in addition $\mu(X)=1$, we say that $\mu$ is a probability measure on $X$.

3. A finitely additive measure is called $\sigma$-additive if $\Omega$ is a $\sigma$-algebra and for every countable family $\left\{A_{i}: i \in \mathbb{N}\right\}$ of disjoint subsets in $\Omega$ we have $\mu\left(\bigcup_{i=1}^{\infty} A_{i}\right)=\sum_{i=1}^{\infty} \mu\left(A_{i}\right)$.

Example 1.2.4. The following are standard examples in measure theory:

- Let $X=\mathbb{N}$ and define $\mu: \mathcal{P}(\mathbb{N}) \rightarrow \mathbb{R}^{\geq 0} \cup\{\infty\}$ as $\mu(A)=|A|$. Then, $\mu$ is a $\sigma$-additive measure. ( $\mu$ is called the counting measure)

- Let $X$ be a non-empty set, and fix an element $p \in X$. Then the function $\mu_{p}$ : $\mathcal{P}(X) \rightarrow\{0,1\}$ defined as :

$$
\mu_{p}(A)= \begin{cases}1 & \text { if } p \in A \\ 0 & \text { otherwise }\end{cases}
$$

is a $\sigma$ additive measure. This measure is known as the measure centered in $p$.

Now we move on to describe the so-called Keisler measures. For a given definable sets $X$ in $\mathcal{M}$, we indicate with $\operatorname{Def}_{A}(X)$ the collection of all definable subsets of $X$ with 
parameters over $A$. We denote by $\operatorname{Def}(X)$ the set $\operatorname{Def}_{\mathcal{M}}(X)$. Also, we refer the reader to [10] for more details.

Definition 1.2.5 (Keisler measures).

1. A Keisler measure $\mu$ on $X$ over $A$ is a probability measure defined on the Boolean algebra $\operatorname{Def}_{A}(X)$.

2. A global Keisler measure is a probability measure defined on $\operatorname{Def}(X)$.

3. If $\mu$ is a Keisler measure on $\operatorname{Def}_{B}(X)$ and $A \subseteq B$, we write $\mu \uparrow_{A}$ for the restriction of $\mu$ to $\operatorname{Def}_{A}(X)$.

Let $\phi(\bar{x}, \bar{y})$ be an $\mathcal{L}$-formula with $\bar{x}$ a tuple of variables with sort $X$. Given a Keisler measure $\mu$ on $\operatorname{Def}(X)$, we can define the function $\mu_{\phi}: \mathcal{M}^{|\bar{y}|} \rightarrow[0,1]$ by $\mu_{\phi}(\bar{a}):=\mu(\phi(\bar{x}, \bar{a}))$. Conversely, a global Keisler measure on $X$ is completely determined by the family of functions $\left\{\mu_{\phi} \mid \phi=\phi(\bar{x}, \bar{y})\right.$ is an $\mathcal{L}$-formula $\}$ and $\mu_{\phi}: S_{\phi} \rightarrow[0,1]$.

Theorem 1.2.6. [Extension of measures] Let $\mu$ a Keisler measure on $X$ over a model $\mathcal{M}_{0}$, then $\mu$ can be extended to a global Keisler measure.

Proof. Let $\mu$ a Keisler measure on $X$ over a model $\mathcal{M}_{0}$, viewed as a map from the definable subsets of $X\left(\mathcal{M}_{0}\right)$ over $\mathcal{M}_{0}$ to $[0,1]$. Let $\mathcal{L}^{\prime}$ be the two-sorted language $\mathcal{L} \cup\{M,(\mathcal{I},+,<$ $\left., 0,1), \mu_{\phi}\right\}$ where the sort $M$ carries the $\mathcal{L}$-structure, $\mathcal{I}$ is a sort of an ordered interval with end points 0 and 1 , and $\mu_{\phi}$ are functions from $M^{|\bar{y}|} \rightarrow \mathcal{I}$. Consider the $\mathcal{L}^{\prime}$-structure $\mathcal{A}=\left\langle\mathcal{M}_{0},[0,1],+,<, \mu_{\phi}\right\rangle$ where:

- $\mathcal{M}_{0}$ is the original structure adding a constant for each element,

- $[0,1]$ is the real interval, with the order and the addition modulo 1 ,

- For each $\mathcal{L}$-formula $\phi(\bar{x}, \bar{y})$, and $\bar{m} \in \mathcal{M}_{0}, \mu_{\phi}(\bar{m}):=\mu(\phi(\bar{x}, \bar{m}))$. 
Let $\mathcal{A}^{\prime}=\left\langle\mathcal{M}^{\prime},[0,1]^{\prime},+,<, \mu_{\phi}^{\prime}\right\rangle$ be a saturated model of $\mathcal{A}$. Let $s t:[0,1]^{\prime} \rightarrow[0,1]$ be the standart part map. We will show that the map st $\circ \mu^{\prime}: \operatorname{Def}(X) \rightarrow[0,1]$ is a global Keisler measure that extends $\mu$.

Firstly, note that any $q \in \mathbb{Q} \cap[0,1]$ is $\mathcal{L}^{\prime}$-definable. Let $\bar{m} \subset \mathcal{M}_{0}$. Since $\mathcal{A}^{\prime}$ is an elementary extension of $\mathcal{A}, \mathcal{A} \models \mu(\phi(\bar{x}, \bar{m})) \leq q$ if and only if $\mathcal{A}^{\prime} \models \mu(\phi(\bar{x}, \bar{m})) \leq q$. Therefore, $\mu_{\phi}^{\prime}(\bar{m}) \in[0,1]^{\prime}$ is an element in the same rational cut as $\mu(\phi(\bar{x}, \bar{m}))$, and by composing with the standard map we have that $\operatorname{st}\left(\mu_{\phi}^{\prime}(\bar{m})\right):=\mu^{\prime}(\phi(\bar{x}, \bar{m}))=\mu(\phi(\bar{x}, \bar{m}))$. Therefore, $\mu^{\prime} \Upsilon_{\mathcal{M}_{0}}=\mu$.

We show now that $\mu^{\prime}$ is a probability measure. Note that $\mu^{\prime}(\emptyset)=\mu(\emptyset)=0$ and $\mu^{\prime}(X)=\mu(X)=1$, since this is a first order property. Let $\phi\left(\bar{x}, \bar{y}_{1}\right)$ and $\psi\left(\bar{x}, \bar{y}_{2}\right)$ be two $\mathcal{M}$-formulas. Again since $\mathcal{A}^{\prime}$ is an elementary extension of $\mathcal{A}$ we have

$$
\begin{gathered}
\mathcal{A} \models \forall \bar{y}_{1} \forall \bar{y}_{2}\left(\forall \bar{z}\left(\neg \phi\left(\bar{z}, \bar{y}_{1}\right) \wedge \phi\left(\bar{z}, \bar{y}_{2}\right)\right) \rightarrow\left(\mu_{\phi}\left(\bar{y}_{1}\right)+\mu_{\psi}\left(\bar{y}_{2}\right)=\mu_{\phi \vee \psi}\left(\bar{y}_{1}, \bar{y}_{2}\right)\right)\right. \\
\Leftrightarrow \mathcal{A}^{\prime} \models \forall \bar{y}_{1} \forall \bar{y}_{2}\left(\forall \bar{z}\left(\neg \phi\left(\bar{z}, \bar{y}_{1}\right) \wedge \phi\left(\bar{z}, \bar{y}_{2}\right)\right) \rightarrow\left(\mu_{\phi}\left(\bar{y}_{1}\right)+\mu_{\psi}\left(\bar{y}_{2}\right)=\mu_{\phi \vee \psi}\left(\bar{y}_{1}, \bar{y}_{2}\right)\right)\right.
\end{gathered}
$$

that is, $\mu^{\prime}: \operatorname{Def}(X) \rightarrow[0,1]^{\prime}$ is finitely additive and since the standard map is linear, we have that st $\circ \mu^{\prime}$ is a finitely additive measure.

Lemma 1.2.7. Let $B=\left\langle\bar{b}_{i} \mid i \in \omega\right\rangle$ be an indiscernible sequence, then we can find a sequence $C=\left\langle\bar{c}_{i} \mid i \in \omega\right\rangle$ such that:

- $C$ satisfies the EM-type of $B$,

- $C$ is $\mu$-indiscernible.

Proof. It follows by the Erdos-Rado theorem and compactness. For a detailed explanation we refer the reader to [15] Lemma 5.13.

Lemma 1.2.8. Let $\mu$ be a Keisler measure on $X$. Let $\bar{x}$ be a variable of sort $X$. Let $\phi(\bar{x}, \bar{y})$ an $\mathcal{L}$-formula and let $\left\langle\bar{b}_{i} \mid i \in \omega\right\rangle$ be an indiscernible sequence such that for some 
$\epsilon>0, \mu\left(\phi\left(\bar{x}, \bar{b}_{i}\right)\right) \geq \epsilon$ for all $i \in \omega$. Then, $\mathcal{F}=\left\{\phi\left(\bar{x}, \bar{b}_{i}\right) \mid i \in \omega\right\}$ is consistent.

Proof. Consider the extended structure $\mathcal{A}=\left\langle\mathcal{M},[0,1], \mu_{\phi}\right\rangle$ induced by the Keisler measure $\mu$ on $X$. Notice that by compactness it is enough to show that $\mathcal{F}$ is finitely consistent, and this condition is preserved by the EM-type of $\left\langle\bar{b}_{i}: i \in \omega\right\rangle$.

By Lemma 1.2.7 we may assume that $B=\left\langle\bar{b}_{i} \mid i \in \omega\right\rangle$ is $\mathcal{L}^{\prime}$-indiscernible. In particular, $B$ is $\mu$-indiscernible.

Let $Y_{b_{i}}$ the set in $\mathcal{M}$ defined by $\phi\left(\bar{x}, \bar{b}_{i}\right)$. By $\mu$-indiscernibility, whenever $i_{1}<\cdots<i_{n}$, and $j_{1}<\cdots<j_{n}$ we have $: \mu\left(Y_{b_{i_{1}}} \cap \cdots \cap Y_{b_{i_{n}}}\right)=\mu\left(Y_{b_{j_{1}}} \cap \cdots \cap Y_{b_{j_{n}}}\right)=r_{n}$ for a fixed $r_{n} \in[0,1]$. We have by hypothesis that $r_{1} \geq \epsilon>0$.

Suppose that there is $n \in \omega$ such that $\mathcal{F}$ is $n$-inconsistent. Then $\bigcap_{i=1}^{n} Y_{b_{i}}=\emptyset$. Choose a maximal $k \in \mathbb{N}$, such that $r_{k}>0$ and for each $j \geq 0$ consider the set $Z_{j}=Y_{b_{1}} \cap \cdots \cap$ $Y_{b_{k-1}} \cap Y_{b_{k+j}}$. We have by $\mu$-indiscernibility that $\mu\left(Z_{j}\right)=r_{k}$ and by maximality of $k$, $\mu\left(Z_{j_{1}} \cap Z_{j_{2}}\right)=\mu\left(Y_{b_{1}} \cap \cdots \cap Y_{b_{k-1}} \cap Y_{b_{k}} \cap Y_{b_{k+1}}\right)=0$ for every $0 \leq j_{1}<j_{2}$. Let $M \in \mathbb{N}$ be such that $M \cdot r_{k}>1$, then $\mu(X) \geq \mu\left(\bigcup_{j=1}^{M} Z_{j}\right)=\sum_{j=1}^{n} \mu\left(Z_{j}\right)=M \cdot r_{k}>1$, a contradiction.

We conclude that $\mathcal{F}=\left\{\phi\left(\bar{x}, \bar{b}_{i}\right) \mid i \in \omega\right\}$ is consistent.

Definition 1.2.9 $\left(\sim_{\mu}\right)$. Let $X$ be a definable set and $\mu$ a Keisler measure over $X$. Let $Y, Z$ two definable subsets of $X$ we define $Y \sim_{\mu} Z$ if and only if $\mu(Y \triangle Z)=0$.

Lemma 1.2.10. $\sim_{\mu}$ is an equivalence relation in $\operatorname{Def}(X)$.

Proof. Since $\mu(X \triangle X)=\mu(\emptyset)=0$, we have that $\sim_{\mu}$ is reflexive. Symmetry comes from the fact that $X \triangle Y=Y \triangle X$. Finally, assume $X \sim_{\mu} Y$ and $Y \sim_{\mu} Z$, and notice that since $X \triangle Z=(X \triangle Y) \triangle(Y \triangle Z)$, we have that $\mu(X \triangle Z) \leq \mu(X \triangle Y)+\mu(Y \triangle Z)=0+0=0$. We conclude that $X \sim_{\mu} Z$. 


\subsubsection{Keisler measures in dependent theories}

In this section we state some theorems of [7], which establish a relationship between the dependence property and the Keisler Measure. This connection will be a crucial point to show that any definable bounded group in Presburger Arithmetic have the fsg property.

Lemma 1.2.11. Assume that $T$ is a dependent theory. Let $\mu$ be a global Keisler measure on $X, \phi(\bar{x}, \bar{y})$ a formula with $\bar{x}$ of sort $X$ and $\epsilon>0$. Then, there is no sequence $\left\langle\bar{b}_{i} \mid i \in \omega\right\rangle$ such that for all $i \neq j, \mu\left(\phi\left(\bar{x}, \bar{b}_{i}\right) \triangle \phi\left(\bar{x}, \bar{b}_{j}\right)\right) \geq \epsilon$.

Proof. Assume for a contradiction that there is such sequence $\left\langle\bar{b}_{i} \mid i \in \omega\right\rangle$. By Lemma 1.2.7, we may assume that $\left\langle\bar{b}_{i} \mid i \in \omega\right\rangle$ is an indiscernible sequence. Thus, the sequence $\left\langle\bar{b}_{i} \bar{b}_{i+1}: i<\omega\right\rangle$ is also indiscernible and since $\mu\left(\phi\left(\bar{x} ; \bar{b}_{i}\right) \triangle \phi\left(\bar{x} ; \bar{b}_{i+1}\right)\right) \geq \epsilon$ for every $i<\omega$, then by Lemma 1.2.8 we have that the set $\left\{\phi\left(\bar{x}, \bar{b}_{2 j}\right) \triangle \phi\left(\bar{x}, \bar{b}_{2 j+1}\right)\right\}$ must be consistent.

However, this contradicts that $T$ is dependent by Corollary 1.1.25.

Corollary 1.2.12. Assume that $T$ is a dependent theory and let $\mu$ be a global Keisler measure on a definable set $X$. Then there are only boundedly many $\sim_{\mu}$-classes of definable subsets of $X$.

In particular there is a small model $\mathcal{M}_{0}$, such that every definable subset $Y$ of $X$ is $\sim_{\mu}$-equivalent to some $\mathcal{M}_{0}$-definable subset $X$.

Proof. Assume for contradiction that there are unboundedly many $\sim_{\mu^{-}}$classes of definable subsets of $X$.

Claim 1: There is a formula $\psi(\bar{x}, \bar{y})$ and a sequence $\left\langle\bar{b}_{i} \mid i \in \omega\right\rangle$ such that for all $i \neq j$

$$
\mu\left(\psi\left(\bar{x}, \overline{b_{i}}\right) \triangle \psi\left(\bar{x}, \overline{b_{j}}\right)\right)>0
$$

Let $\mathcal{C}=\left\{[\phi(\bar{x}, \bar{b})]_{\sim_{\mu}} \mid \bar{b} \subseteq \mathcal{M}, \phi(\bar{x} ; \bar{y}) \in \mathcal{L}\right\}$ be the collection of all $\sim_{\mu}$-classes, and consider the function $f: \mathcal{L} \times \mathcal{P}^{<\omega}(\mathcal{M}) \rightarrow \mathcal{C}$ given by $f(\phi(\bar{x}, \bar{y}) ; \bar{b}) \mapsto[\phi(\bar{x} ; \bar{b})]_{\sim_{\mu}}$ This is 
a surjective function, and since $\mathcal{C}$ is unbounded, $\kappa=|\mathcal{C}|>|T|$. By pigeonhole principle there is a formula $\psi(\bar{x}, \bar{y})$ such that the collection $\left\{[\psi(\bar{x} ; \bar{b})]_{\sim_{\mu}}: \bar{b} \subseteq \mathcal{M}\right\}$ contains infinitely many $\sim_{\mu}$-classes. Then, we can choose an infinite sequence $\left\langle\bar{b}_{i} \mid i \in \omega\right\rangle$ such that for all $i \neq j, \mu\left(\psi\left(\bar{x}, \overline{b_{i}}\right) \triangle \psi\left(\bar{x}, \overline{b_{j}}\right)\right)>0$.

$\square_{\text {Claim 1. }}$.

By Lemma 1.2.7, there is a $\mu$-indiscernible sequence $\left\langle\bar{d}_{i}: i<\omega\right\rangle$ with the same $\mu$-EMtype of $\left\langle\bar{b}_{i}: i<\omega\right\rangle$. Observe that by $\mu$-indiscernibility, $\mu\left(\psi\left(\bar{x}, \bar{d}_{i}\right) \triangle \psi\left(\bar{x}, \bar{d}_{j}\right)\right)=\epsilon>0$ is constant for every $i<j<\omega$. This contradicts Lemma 1.1.25, and we conclude that the $\sim_{\mu}$-classes of the definable subsets of $X$ are bounded.

Consider now a set $H=\left\{\phi_{\alpha}\left(\bar{x}, \bar{b}_{\alpha}\right) \mid \alpha \in \lambda\right\}$ of representatives for the equivalence relation $\sim_{\mu}$. Since there are only boundedly many $\sim_{\mu}$-classes, we may assume that $\lambda<$ $|\mathcal{M}|$. Let $A=\bigcup_{\alpha<\lambda} \bar{b}_{\alpha}$, and notice that $|A| \leq \lambda \cdot \omega=\lambda$. By downward LöwenheimSkolem, there is a model $\mathcal{M}_{0} \prec \mathcal{M}$ of size $\lambda$ containing $A$. Notice that $\mathcal{M}_{0}$ is a small model, and by construction, for any definable subset $Y$ of $X$, there is an $\mathcal{M}_{0}$-definable set $Z$, such that $Y \sim_{\mu} Z$.

\subsubsection{Groups with fsg}

In this section we recall several results presented in [7]. In this paper Hrushovski, Peterzil and Pillay presented a property called the fsg (finitely satisfiable generics), and they use this property to characterize the subgroup $G^{00}$ as the stabilizer of a generic global type.

Definition 1.2.13. $\quad 1$. Let $(G, \cdot, e)$ be a definable group. We say that a type $p(\bar{x})$ is centered in $G$ if $p(\bar{x}) \models(x \in G)$.

2. Given $g \in G$ and a type $p(x)$ centered in $G$, we define the left-translate of $p$ by $g$ to be the type $g \cdot p:=\left\{\phi(x): \phi\left(g^{-1} x\right) \in p(x)\right\}$.

Notice that if $p(\mathcal{M})$ is the set of realizations of $p(x)$, then $g \cdot p(\mathcal{M})$ corresponds with the set of realizations of the type $g \cdot p$. 
Definition 1.2.14 (Generics). Let $X$ be a definable subset of a definable group $(G, \cdot, e)$, and $p(\bar{x})$ a type centered in $G$.

1. We say that $X$ is left-generic (resp. right-generic) if finitely many left- translates (resp. right-translates) of $X$ cover $G$. Namely, there are $g_{1}, \cdots, g_{k} \in G$ such that $G=\bigcup_{i=1}^{k} g_{i} \cdot X$

2. If $X$ is both left-generic and right-generic, we will say that $X$ is generic.

3. We say that $p(\bar{x})$ is a generic type if all its formulas are generic.

Example 1.2.15. The following are examples of generic sets in definable groups:

Let $T$ be any theory and $G$ be a definable group of $T$. Clearly, $X=G$ is generic.

Let $T$ be an extension of the o-minimal theory $T h(\mathbb{R},+, \cdot,<, 0,1)$ and let $\mathcal{M}$ be a very saturated model of $T$. Any cofinite subset of a definable group $G$ in $T$ is generic.For example, consider the definable group $S_{1}=\left\{(x, y) \mid x^{2}+y^{2}=1\right\}$, the set $S_{1} \backslash\{1\}$ is generic.

Definition 1.2.16. [A group with fsg] Let $\left(G, \cdot, e_{G}\right)$ be a definable group. We say $G$ has finitely satisfiable generics ( $f s g$ ) if there exists a small elementary submodel $\mathcal{M}_{0}$ and a global generic type $p(x) \in S(\mathcal{M})$, such that $p(x) \models x \in G$, and for every $g \in G(\mathcal{M})$, the left-translate $g \cdot p(x):=\left\{\phi(\bar{x}) \mid \phi\left(g^{-1} \cdot \bar{x}\right) \in p(\bar{x})\right\}$ of $p(\bar{x})$ is finitely satisfiable in $\mathcal{M}_{0}$.

Fact 1.2.17. Let $p(x)$ be a complete global type centered in $G$ and assume that $X$ is generic. Then there is some $g \in G$ such that $X \in g \cdot p$.

Proof. Since $X$ is generic, there are $g_{1}, \cdots, g_{k} \in G$ such that $G=\bigcup_{i=1}^{k}\left(g_{i} \cdot X\right)$. Let $\phi(\bar{x}, \bar{b})$ be a formula which defines $G$, and $\psi(\bar{y}, \bar{a})$ the formula which defines $X$. Notice that:

$$
\mathcal{M} \models \forall \bar{x}\left(\phi(\bar{x}, \bar{b}) \leftrightarrow \bigvee_{i=1}^{k}\left(\exists \bar{y}\left(\psi(\bar{y}, \bar{a}) \wedge x=g_{i} \cdot \bar{y}\right)\right)\right)
$$


Since $\phi(x, \bar{m}) \in p(\bar{x})$, then $\bigvee_{i=1}^{k} g_{i} \cdot X \in p$, and by completeness of $p$ there is some $i \leq k$, such that $g_{i} \cdot X \in p$. Therefore $X \in g_{i}^{-1} \cdot p(\bar{x})$.

Lemma 1.2.18. Assume that $G$ has fsg, witnessed by $p(\bar{x})$ and $\mathcal{M}_{0}$. Let $X$ be a definable subset of $G$, then:

1. $X$ is left-generic if and only if $X$ is right-generic, then we say simply that $X$ is generic.

2. $X$ is generic if and only if every left (right)-translate of $X$ meets $\mathcal{M}_{0}$.

3. The type $p(\bar{x})$ is generic, as well as any other right or left translate of $p(\bar{x})$.

4. If $X$ is generic and $X=X_{1} \cup X_{2}$, where $X_{1}$ and $X_{2}$ are definable, then one of them is generic.

Proof. $\quad$ 1. Assume that $X$ is left-generic, then every translate of $X$ is also left-generic. Let $c$ be an arbitrary element of $G$. By Fact 1.2.17, there is some $g \in G$, such that $c \cdot X \in g \cdot p(\bar{x})$. By fsg, $(c \cdot X) \cap G\left(\mathcal{M}_{0}\right) \neq \emptyset$. Let $b \in(c \cdot X) \cap G\left(\mathcal{M}_{0}\right)$, then there is some $x \in X$, such that $b=c \cdot x$, and so $c^{-1}=x \cdot b^{-1} \in X \cdot b^{-1}$. Since $b^{-1} \in G\left(\mathcal{M}_{0}\right)$, we have shown that for any element $c \in G$, there is an element $b \in G\left(\mathcal{M}_{0}\right)$ such that $c \in X \cdot b$.

If $X$ were not right-generic, then for any $h_{1}, \cdots, h_{n} \in G\left(\mathcal{M}_{0}\right)$ there is an element $c \in G$ such that $c \notin \bigcup_{i=1}^{n} X \cdot h_{i}$. Let $\phi(\bar{x}, \bar{m})$ and $\psi(\bar{x}, \bar{a})$ be the formulas defining $G$ and $X$, respectively. Consider the type $\sigma(\bar{z})=\{\phi(\bar{z}, \bar{m})\} \cup\{\forall \bar{x}(\psi(\bar{x}, \bar{m}) \rightarrow \neg(\bar{c}=$ $\left.\bar{x} \cdot h)) \mid h \in G\left(\mathcal{M}_{0}\right)\right\}$. Notice $\Sigma(\bar{c})$ is finitely consistent because $X$ is not right generic. By saturation of $\mathcal{M}$ there is a tuple $\bar{c}$ such that $\mathcal{M} \models \Sigma(\bar{c})$, then $\bar{c} \in G$, and $\forall h \in G\left(\mathcal{M}_{0}\right), \bar{b} \notin X \cdot h$, which contradicts the result obtaining in the previous paragraph. We conclude then that $X$ is right generic. The other direction follows in the same way. 
2. It follows directly from the previous argument.

3. By hypothesis, for every $X \in p(\bar{x})$, and every element $g \in G$, we have $(g \cdot X) \cap$ $G\left(\mathcal{M}_{0}\right) \neq \emptyset$. By $(2), X$ is generic. Since every formula in $p(\bar{x})$ is generic, we conclude that $p(\bar{x})$ is a generic type.

4. Let $X_{1}, X_{2}$ two definable subsets of $G$ and assume that $X=X_{1} \cup X_{2}$ is generic. By Fact 1.2.17, there is an element $g \in G$ such that $X \in g \cdot p(\bar{x})$, and since $p(\bar{x})$ is complete, either $X_{1} \in g \cdot p(\bar{x})$ or $X_{2} \in g \cdot p(\bar{x})$. Thus, either $X_{1}$ or $X_{2}$ is generic.

Remark 1.2.19. Notice that (4) implies that the collection $\mathcal{I}_{G}=\{Y \subset G \mid Y$ is nongeneric in $G$ \} is an ideal in the algebra of the definable subsets of $G$.

Definition 1.2.20 $\left(\sim_{I}\right)$. Let $(G, \cdot, e)$ be a definable group. We define the relation $\sim_{I}$ on $\operatorname{Def}(G)$ given by $X \sim_{I} Y$ if and only if $X \triangle Y$ is non-generic.

Lemma 1.2.21. If $\mathcal{I}_{G}$ is an ideal, then the relation $\sim_{I}$ is an equivalence relation.

Proof. Note that $X \triangle X=\emptyset$, which is non-generic. So, $\sim_{I}$ is reflexive. Symmetry follows from the fact that $X \triangle Y=Y \triangle X$.

To show transitivity, suppose that $X \sim_{I} Y$ and $Y \sim_{I} Z$, then $X \triangle Y$ and $Y \triangle Z$ is non-generic. Since $X \triangle Z \subseteq(X \triangle Y) \cup(Y \triangle Z)$ and both $(X \triangle Y),(Y \triangle Z)$ are non-generic, the fact that $\mathcal{I}_{G}$ is an ideal implies that $X \triangle Z$ is non-generic. Thus, $X \sim_{I} Z$.

Definition 1.2.22 (Subgroup of bounded index). Let $G$ be a definable group in $\mathcal{M}$ and let $H$ be a subgroup of $G$. We say that it has bounded index if $[G(\mathcal{M}): H]=\alpha<\kappa$, where $\kappa=|\mathcal{M}|$. (In particular $|\mathcal{M}| \geq 2^{|T|}$ )

Definition 1.2.23. Let $(G, \cdot, e)$ be a definable group. We say that $G$ is definably amenable if there exists a Keisler measure on $\operatorname{Def}(G)$ that is $G$-invariant.

Lemma 1.2.24. Suppose that $T$ is a dependent theory and let $G$ be a group definable in $T$ that is definably amenable and such that the non-generic sets form an ideal. 
1. There is only a bounded number of definable subsets of $G$ modulo the equivalence relation $X \sim_{I} Y$.

2. For each definable generic subset $X \subset G$, the stabilizer of $X$ in $G$, defined as: $\operatorname{Stab}_{\mathcal{I}}(X):=\{g \in G \mid g \cdot X \triangle X$ is non-generic $\}$ is a type definable subgroup of bounded index.

Proof. 1. Observe that if $X$ is generic then $\mu(X)>0$. Indeed, if $g_{1}, \cdots, g_{k}$ are elements ni $G$ such that $G=\bigcup_{i=1}^{k} g_{i} \cdot X$, then

$$
1=\mu(G)=\mu\left(\bigcup_{i=1}^{k} g_{i} \cdot X\right) \leq \sum_{i=1}^{k} \mu\left(g_{i} \cdot X\right)=\sum_{i=1}^{k} \mu(X)=k \mu(X)
$$

and we have $\mu(X) \geq \frac{1}{k}>0$. So, given two definable subsets $Y, Z \subseteq X$ such that $Y \chi_{I} Z$, we have that $Y \triangle Z$ is generic, and $\mu(Y \triangle Z)>0$. Thus, $Y \chi_{\mu} Z$. Hence, if there are unboundedly many $\sim_{I^{-}}$classes, there are also unboundedly many $\sim_{\mu^{-}}$ classes, and this contradicts Lemma 1.2.12.

2. Let $X$ be a generic set. We will see that $\operatorname{Stab}_{\mathcal{I}}(X)=\{g \in G \mid g \cdot X \triangle X$ is nongeneric $\}$ is a type definable subgroup of $G$ of bounded index.

First we show that $\operatorname{Stab}_{\mathcal{I}}(X)$ is a subgroup of $G$. Notice that $e \in \operatorname{Stab}_{\mathcal{I}}(X)$ since $e \cdot X \triangle X=X \triangle X=\emptyset$, which is non-generic. Also, if $g \in \operatorname{Stab}_{\mathcal{I}}(X)$, then $(g \cdot X) \triangle X$ is non-generic, and $\left(g^{-1} \cdot X\right) \triangle X=g^{-1}(X \triangle(g \cdot X))$ is also non-generic because it is the translate of a non-generic set. So, $g^{-1} \in \operatorname{Stab}_{\mathcal{I}}(X)$.

Suppose now that $g_{1}, g_{2} \in \operatorname{Stab}_{\mathcal{I}}(X)$, we want to show that $\left(g_{2} \cdot g_{1} \cdot X\right) \triangle X$ is non-generic. Observe that:

$$
\begin{aligned}
\left(g_{2} \cdot g_{1} \cdot X\right) \triangle X & =g_{2} \cdot\left(\left(g_{1} \cdot X\right) \triangle\left(g_{2}^{-1} \cdot X\right)\right) \\
& =g_{2} \cdot\left(\left(\left(g_{1} \cdot X\right) \triangle X\right) \triangle\left(\left(g_{2}^{-1} \cdot X\right) \triangle X\right)\right)
\end{aligned}
$$

and the sets $Y_{1}=\left(\left(g_{1} \cdot X\right) \triangle X\right) \backslash\left(\left(g_{2}^{-1} \cdot X\right) \triangle X\right)$ and $Y_{2}=\left(\left(g_{2}^{-1} \cdot X\right) \triangle X\right) \backslash\left(\left(g_{1}\right.\right.$. 
$X) \triangle X)$ are non-generic, because they are subsets of the non-generic sets $\left(g_{1} \cdot X \triangle X\right)$ and $\left(g_{2} \cdot X \triangle X\right)$, respectively. Since $\mathcal{I}_{G}$ is an ideal, we conclude that $Y_{1} \cup Y_{2}$ is nongeneric, and finally $g_{2} \cdot\left(Y_{1} \cup Y_{2}\right)=\left(g_{2} \cdot g_{1} \cdot X\right) \triangle X$ is also non-generic.

We show now that $\operatorname{Stab}_{\mathcal{I}}(X)$ is type definable. For each $n \in \omega$ consider the formula

$$
\phi(g)=\forall h_{1} \cdots h_{n}\left(\bigwedge_{i=1}^{n}\left(h_{i} \in G\right) \rightarrow\left(\exists c\left(c \in G \wedge \bigwedge_{i=1}^{n} c \notin\left(h_{i} \cdot(g \cdot X \triangle X)\right)\right)\right)\right)
$$

and let $A_{n}$ be the set of points in $\mathcal{M}$ defined by $\phi(g)$. Observe that $\operatorname{Stab}_{\mathcal{I}}(X)=\bigcap_{n \in \omega} A_{n}$, therefore it is a type-definable set.

We finally show that $\operatorname{Stab}_{\mathcal{I}}(X)$ has bounded index in $G$. Observe that $g_{1} \cdot \operatorname{Stab}_{\mathcal{I}}(X) \neq$ $g_{2} \cdot \operatorname{Stab}_{\mathcal{I}}(X)$, if and only if $g_{2}^{-1} \cdot g_{1} \notin \operatorname{Stab}_{\mathcal{I}}(X)$ if and only if $\left(g_{2}^{-1} \cdot g_{1} \cdot X\right) \triangle X$ is generic, which implies $\left(g_{1} \cdot X\right) \triangle\left(g_{2} \cdot X\right)$ is generic, and thus $\left(g_{1} \cdot X\right) \chi_{\mathcal{I}}\left(g_{2} \cdot X\right)$. Since, there are only boundedly many $\sim_{\mathcal{I}^{-}}$classes, then there is also a bounded number of cosets of $\operatorname{Stab}_{\mathcal{I}}(X)$ in $G$.

Definition 1.2.25. Let $A$ be a small set of parameters and $G$ be a definable group. We define $G_{A}^{00}$ as the smallest type $A$-definable subgroup of bounded index of $G$.

Remark 1.2.26. Shelah proved that if $G$ is definable in a dependent theory, then for every set of parameters $A$ we have that $G_{A}^{00}=G_{\emptyset}^{00}$. Then, since Presburger Arithmetic is a dependent theory, we will refer to the smallest type-definable subgroup of $G$ just as $G^{00}$.

Definition 1.2.27 (Stabilizer of a type). Let $p$ be a global type, then

$$
\operatorname{Stab}(p)=\{g \in G \mid g \cdot p=p\}
$$

Remark 1.2.28. For every global type $p$, we have that $\operatorname{Stab}(p)$ is a subgroup of $G$.

Theorem 1.2.29. Assume that $G$ has fsg. Then: 
1. There is a bounded of number of global generic types.

2. $G^{00}$ exists.

3. For each global generic type $q, G^{00}=\bigcap_{X \in q} \operatorname{Stab}_{\mathcal{I}}(X)=\operatorname{Stab}(p)$.

Proof. 1. Let $\mathcal{G G} \mathcal{T}$ be the set of the generic global types centered in $G$, and consider the function $\mathcal{S}: \mathcal{G G} \mathcal{T} \rightarrow \mathcal{P}\left(\mathcal{P}\left(\mathcal{M}_{0}\right)\right)$, defined as $\mathcal{S}(p)=\left\{X \cap G\left(\mathcal{M}_{0}\right) \mid X \in p\right\}$. Since $\left|\mathcal{P}\left(\mathcal{P}\left(\mathcal{M}_{0}\right)\right)\right|=2^{2^{\mathcal{M}_{0}}}$ is bounded, it is enough to show that $\mathcal{S}$ is injective.

Let $p, q$ be two different generic global complete types, and take a formula $\phi(\bar{x}) \in p$ such that $\neg(\phi(\bar{x})) \in q$. Let $A=\phi(\mathcal{M}) \cap G\left(\mathcal{M}_{0}\right)$ and notice that $A \in \mathcal{S}(p)$, we will show that $A \notin \mathcal{S}(q)$. Suppose by contradiction, that $A \in \mathcal{S}(q)$. Then there is a formula $\psi(\bar{x}) \in q$, such that $\psi(\mathcal{M}) \cap G\left(\mathcal{M}_{0}\right)=A=\phi(\mathcal{M}) \cap G\left(\mathcal{M}_{0}\right)$, but then $(\psi(\mathcal{M}) \cap \neg \phi(\mathcal{M})) \cap G\left(\mathcal{M}_{0}\right)=\emptyset$. So, the set defined by the formula $\neg \phi(x) \wedge \psi(x)$ in $\mathcal{M}$ does not meet $\mathcal{M}_{0}$, and by Lemma 5.3(2) this implies that the set defined by $\neg \phi(x) \wedge \psi(x)$ is not generic, contradicting that $q$ is generic.

Therefore, the function $\mathcal{S}$ is injective, and $|\mathcal{G G T}| \leq 2^{2^{\left|\mathcal{M}_{0}\right|}}$. We conclude that there are only boundedly many global generic types.

2. Using the notation from (1), let $|\mathcal{G G T}|=\lambda$ be the (bounded) cardinality of the collection of global generic types. Let $H$ be a type-definable subgroup of $G$ with bounded index in $G$ and let $p$ be some global fixed generic type.

Claim: Each coset of $H$ is in some translate of $p$.

Assume that $H$ is defined by $\bigwedge\left(\phi_{i}\left(\bar{x}, \bar{a}_{i}\right)\right)$, where each $\bar{a}_{i}$ is a tuple from $\mathcal{M}$. Since $H$ has bounded index in $G,|\{g \cdot H \mid g \in G\}|=\gamma<|\mathcal{M}|$. Let $\left\{g_{j} \cdot H\right\}_{j<\gamma}$ be a set of representatives of $G / H$.

For a contradiction, let us assume that for all $g_{j} \in G, g_{j} \cdot H \notin p$. Then, for every $j<\gamma$ there is some $i_{j}<\alpha$ such that $g_{j} \cdot \phi_{i_{j}}\left(\bar{x}, \bar{a}_{i_{j}}\right) \notin p$, and since $p$ is complete, 
$\neg\left(g_{j} \cdot \phi_{i_{j}}\left(\bar{x}, \bar{a}_{i_{j}}\right)\right) \in p$.

Consider the type $\Gamma(y)=\{y \in G\} \cup\left\{\neg\left(g_{j} \cdot \phi_{i_{j}}\left(\bar{x}, \bar{a}_{i_{j}}\right)\right) \mid j<\gamma\right\}$, which is consistent since it is contained in $p$. By saturation, there is an element $b \in \mathcal{M}$ realizing $\Gamma(y)$, that is, an element $b$ such that $b \in G$ and

$$
b \notin \bigcup_{j<\gamma}\left(g_{j} \cdot \phi_{i_{j}}\left(\bar{x}, \bar{a}_{i_{j}}\right)\right) \supseteq \bigcup_{j<\gamma} g_{j} \cdot H=G,
$$

which is clearly a contradiction.

Now, by the claim above, we have $|G / H|<\lambda$, since for every $g \in G, g \cdot p$ is also a generic global type. Since this bound is independent from the monster model, we conclude that $G^{00}$ exists. (In fact, let

$$
\mathcal{H}=\{H \subseteq G \mid H \text { is type definable and }|G / H| \leq \lambda\}
$$

and notice that $G^{00}=\bigcap_{H \in \mathcal{H}} H$. Since this intersection is a bounded intersection of subgroups of bounded index it also has bounded index in $G$. It means that $G^{00}$ exists).

3. Fix a gobal generic type $q$, such that $q \models(x \in G)$. Notice that for every definable set $X \in q, X$ is generic, then by Lemma $1.2 .24(2), G^{00} \subseteq \operatorname{Stab}_{\mathcal{I}}(X)$. Therefore $G^{00} \subseteq \bigcap_{X \in q} \operatorname{Stab}_{\mathcal{I}}(X)$

Claim 1: Let $X \in q$ and $g \in \operatorname{Stab}_{\mathcal{I}}(X)$, then $g \cdot X \in q$

Let $X \in q$ and $g \in \operatorname{Stab}_{\mathcal{I}}(X)$, then $(g \cdot X) \triangle X$ is non-generic. Since $X$ is generic and $X=(X \backslash g \cdot X) \cup(X \cap(g \cdot X))$, then $X \cap g \cdot X$ is generic (because $\mathcal{I}_{G}$ is an ideal and $(X \backslash(g \cdot X))$ is non-generic). Additionally, since $X \in q$, by completeness, then $(X \backslash g \cdot X) \in q$ or $X \cap(g \cdot X) \in q$, then $X \cap(g \cdot X) \in q$, therefore $g \cdot X \in q$. 
Claim 2: $\bigcap_{X \in q} \operatorname{Stab}_{\mathcal{I}}(X) \subseteq \operatorname{Stab}(q)$

Let $g \in \bigcap_{X \in q} \operatorname{Stab}_{I}(X)$, then by the step 1, we know that for every definable set $X \in q, g \cdot X \in q$. Then, $g \in \operatorname{Stab}(q)$.

Claim 3: $\operatorname{Stab}(q) \subseteq G^{00}$

Fix an element $h \in \operatorname{Stab}(q)$. Since $G^{00}$ has bounded index, then there is some element $g \in G$, such that $(g \cdot q) \subseteq G^{00}$, it means that the translate $C=g^{-1} \cdot G^{00}$ is in $q$. Therefore, $h \cdot g^{-1} \cdot G^{00}=g^{-1} \cdot G^{00}$, which implies that $h \in g^{-1} \cdot G^{00} \cdot g \subseteq G^{00}$ (because it is a normal subgroup), then $h \in G^{00}$ as we desired.

Summarizing we have that $G^{00} \subseteq \bigcap_{X \in q} \operatorname{Stab}_{\mathcal{I}}(X) \subseteq \operatorname{Stab}(q) \subseteq G^{00}$, therefore all these sets are equal.

\subsection{Amenable Groups}

The notion of definably amenable group presented in the subsection Section 1.2.3 can be strengthened to a property called amenability. Amenable groups have been a topic of study during a long time. There are some relevant theorems in this area, such as every abelian group is amenable, and moreover that every abelian by finite group is also amenable. These results will play a key role in our study of the definable groups in Presburger Arithmetic, and this is for this reason why we present a detailed explanation of these topics in this section. The theory of amenable groups can be found by the reader in $[11]$.

Definition 1.3.1 (Amenable Group). Let $(G, \cdot, e)$ be a group. We will say that $G$ is amenable if there exists $\mu \in[0,1]^{\mathcal{P}(G)}$ such that: 
1. The function $\mu$ is a finitely additive measure,

2. The function $\mu$ is $G$-left-invariant, that is, for all $g \in G$, and $A \subseteq G, \mu(A)=\mu(g \cdot A)$,

3. $\mu(G)=1$.

Lemma 1.3.2. The following statements hold:

1. Every finite group is amenable.

2. If $G$ is an amenable group, and $H$ is a subgroup of $G$ then $H$ is also amenable.

3. If $G$ is an amenable group and $N$ is a normal subgroup of $G$, then $G / N$ is also amenable.

4. Let $G$ be a group and $N$ a normal subgroup of $G$ such that $N$ and $G / N$ are amenable. Then $G$ is amenable.

Proof. 1. Let $(G, \cdot, e)$ be a finite group. Assume that $|G|=n$, then define $\mu: \mathcal{P}(G) \rightarrow$ $[0,1]$ as $\mu(A)=\frac{|A|}{n}$.

- $\mu$ is a finitely additive measure: Notice that $\mu(\emptyset)=\frac{0}{n}=0$, and if $A, B \subset G$ are disjoint, then $|A \cup B|=|A|+|B|$. So, $\mu(A \cup B)=\frac{|A \cup B|}{n}=\frac{|A|+|B|}{n}=$ $\mu(A)+\mu(B)$.

- $\mu$ is G-invariant: Let $g \in G$ and consider $f_{g}: G \rightarrow G$ defined as $f_{g}(x)=g \cdot x$. Note that $f_{g}$ is injective: if $f_{g}\left(x_{1}\right)=f_{g}\left(x_{2}\right)$ then $g \cdot x_{1}=g \cdot x_{2}$ which implies that $x_{1}=x_{2}$ by left cancelation. Thus, for all $A \subset G$ we have $|g \cdot A|=\mid\left\{f_{g}(a)\right.$ : $a \in A\}|=| A \mid$, and therefore $\mu(g \cdot A)=\mu(A)$.

- $\mu(G)=\frac{n}{n}=1$

We conclude that $G$ is amenable.

2. Let $\mu$ be the finitely additive measure witnessing that $G$ is amenable. Let $M$ be a set of representatives for the collection of the right cosets of $H$ in $G$. Consider the function $\nu: \mathcal{P}(H) \rightarrow[0,1]$ defined by $\nu(A)=\mu(\bigcup\{A \cdot g \mid g \in M\})$. 
- $\nu$ is a finitely additive measure: Notice that $\nu(\emptyset)=\mu(\emptyset)=0$ and $\nu(H)=$ $\mu(\bigcup\{H \cdot g \mid g \in M\})=\mu(G)=1$. Consider now $A, B$ disjoint subsets of $H$. If $g_{1}, g_{2} \in M$ and $g_{1} \neq g_{2}$ then $g_{1} \cdot A \cap g_{2} \cdot B=\emptyset$, since they are contained in different cosets. Therefore, it is enough to show that $g \cdot A \cap g \cdot B=\emptyset$.

If $g \cdot A \cap g \cdot B \neq \emptyset$, then there exist $a \in A$ and $b \in B$ such that $g \cdot a=g \cdot b$, and we can conclude that $a=b$, which contradicts the fact that $A$ and $B$ are disjoint.

Hence, $\bigcup\{A \cdot g \mid g \in M\} \cap \bigcup\{B \cdot g \mid g \in M\}=\emptyset$, and we have

$$
\begin{aligned}
\nu(A \cup B) & =\mu(\bigcup\{(A \cup B) \cdot g \mid g \in M\}) \\
& =\mu(\bigcup\{(A \cdot g \cup B \cdot g \mid g \in M\}) \\
& =\mu(\bigcup\{(A \cdot g \mid g \in M\} \cup \bigcup\{(B \cdot g \mid g \in M\}) \\
& =\mu((\bigcup\{(A \cdot g \mid g \in M\})+\mu((\bigcup\{(B \cdot g \mid g \in M\})=\nu(A)+\nu(B)
\end{aligned}
$$

- $\nu$ is $H$ - invariant: Let $h \in H$ and $A \subseteq H$. Then,

$$
\begin{aligned}
\nu(h \cdot A) & =\mu(\bigcup\{((h \cdot A) \cdot g \mid g \in M\})=\mu(\bigcup\{h \cdot(A \cdot g) \mid g \in M\}) \\
& =\mu(h \cdot \bigcup\{(A \cdot g) \mid g \in M\})=\mu(\bigcup\{(A \cdot g) \mid g \in M\}) \\
& =\nu(A) .
\end{aligned}
$$

Thus $H$ is amenable.

3. Let $\mu$ be a measure witnessing that $G$ amenable and $N$ a normal subgroup of $G$. Consider $\nu: \mathcal{P}(G / N) \rightarrow[0,1]$ defined as $\nu(A)=\mu(\bigcup A)$.

- $\nu$ is a finitely additive measure on $G / N$ :

Notice that $\nu(G / N)=\mu(\bigcup G / N)=\mu(G)=1$ and $\nu(\emptyset)=\mu(\emptyset)=0$. Let now $A, B$ be disjoint subsets of $G / N$. We claim that $\bigcup A \cap \bigcup B=\emptyset$. 
If $z \in \bigcup A \cap \bigcup B$ then there exist $g_{1}, g_{2} \in G$ and $n_{1}, n_{2} \in N$ such that $z=$ $g_{1} \cdot n_{1} \in \bigcup A$ and $z=g_{2} \cdot n_{2} \in \bigcup B$. So $g_{2}=g_{1} \cdot n$ for $n=n_{1} \cdot n_{2}^{-1} \in N$ and $g_{1} \cdot N=g_{2} \cdot N \in A \cup B$ contradicting that $\left.A \cap B=\emptyset\right)$.

Then $\nu(A \cup B)=\mu(\bigcup(A \cup B))=\mu(\bigcup A \cup \bigcup B)=\mu(\bigcup A)+\mu(\bigcup B)=$ $\nu(A)+\nu(B)$, as desired.

- $\nu$ is $G / N$ invariant:

Let $g \cdot N \in G / N$ and $A \subseteq G / N$, say $A=\left\{g_{i} \cdot N: i \in I\right\}$. Then, $(g \cdot N) A=$ $\left\{\left(g \cdot g_{i}\right) N: i \in I\right\}$ and hence

$$
\begin{aligned}
\nu((g \cdot N) A) & =\mu\left(\bigcup_{i \in I}\left(g \cdot g_{i}\right) N\right)=\mu\left(\left\{g \cdot g_{i} \cdot n \mid n \in N, i \in I\right\}\right) \\
& =\mu\left(g \cdot\left\{g_{i} \cdot n \mid n \in N, i \in I\right\}\right)=\mu\left(\left\{g_{i} \cdot n \mid n \in N, i \in I\right\}\right) \\
& =\mu\left(\bigcup_{i \in I} g_{i} \cdot N\right)=\mu(\bigcup A)=\nu(A) .
\end{aligned}
$$

Therefore, $G / N$ is amenable.

4. Let $\nu_{1}$ and $\nu_{2}$ be the left-invariant measures of $N$ and $G / N$ respectively, witnessing amenability of these groups. For any $A \subseteq G$, consider $f_{A}: G \rightarrow \mathbb{R}^{\geq 0}$ defined by $f_{A}(g)=v_{1}\left(N \cap g^{-1} \cdot A\right)$. Assume that $g_{1} \cdot N=g_{2} \cdot N$, then there is an $n \in N$ such that $g_{2}^{-1} \cdot g_{1}=n$. Then $g_{2}^{-1}=n \cdot g_{1}^{-1}$, and $f_{A}\left(g_{2}\right)=\nu_{1}\left(N \cap g_{2}^{-1} \cdot A\right)=\nu_{1}\left(N \cap n \cdot g_{1}^{-1} \cdot A\right)=$ $\nu_{1}\left(n \cdot\left(N \cap g_{1}^{-1} \cdot A\right)\right)=\nu_{1}\left(N \cap g_{1}^{-1} \cdot A\right)=f_{A}\left(g_{1}\right)$. Then $f_{A}$ may be regarded as a bounded real-valued function with domain $G / N$, consider $\hat{f}_{A}: G / N \rightarrow \mathbb{R}$ defined as $\hat{f}_{A}(g \cdot N)=f_{A}(g)$. Finally, define $\mu(A)=\int_{G / N} \hat{f}_{A} d \nu_{2}$.

- $\mu$ is a finitely additive measure: We have

$$
\mu(G)=\int_{G / N} \hat{f}_{G} d \nu_{2}=\int_{G / N} 1 d \nu_{2}=\nu_{2}(G / N)=1
$$

and $\mu(\emptyset)=\int_{G / N} \hat{f}_{\emptyset} d \nu_{2}=\int_{G / N} 0 d \nu_{2}=0$. 
Consider now $A, B \subseteq G$ such that $A \cap B=\emptyset$. Notice that $\hat{f}_{A \cup B}=\hat{f}_{A}+\hat{f}_{B}$, since for all $g \in G$,

$$
\begin{aligned}
\hat{f}_{A \cup B}(g) & =f_{A \cup B}(g)=\nu_{1}\left(N \cap g^{-1} \cdot(A \cup B)\right) \\
& =\nu_{1}\left(\left(N \cap g^{-1} \cdot A\right) \cup\left(N \cap g^{-1} \cdot B\right)\right) \\
& =\nu_{1}\left(N \cap g^{-1} \cdot A\right)+\nu_{1}\left(N \cap g^{-1} \cdot B\right) \\
& =f_{A}(g)+f_{B}(g)=\hat{f}_{A}(g)+\hat{f}_{B}(g) .
\end{aligned}
$$

Therefore,

$$
\begin{aligned}
\mu(A \cup B) & =\int_{G / N} \hat{f}_{A \cup B} d \nu_{2}=\int_{G / N} \hat{f}_{A}+\hat{f}_{B} d \nu_{2} \\
& =\int_{G / N} \hat{f}_{A} d \nu_{2}+\int_{G / N} \hat{f}_{B} d \nu_{2}=\mu(A)+\mu(B)
\end{aligned}
$$

as desired.

- $\mu$ is G-invariant: Let $g \in G$ and $A \subset G$, notice that:

$$
\begin{aligned}
\hat{f}_{g \cdot A}(h \cdot N) & =f_{g \cdot A}(h)=\nu_{1}\left(N \cap h^{-1} \cdot g \cdot A\right) \\
& =\nu_{1}\left(N \cap\left(g^{-1} \cdot h\right)^{-1} \cdot A\right)=f_{A}\left(g^{-1} \cdot h\right) \\
& =\hat{f}_{A}\left(g^{-1} \cdot h \cdot N\right)
\end{aligned}
$$

This implies that $\int_{G / N} \hat{f}_{g \cdot A} d \nu_{2}=\int_{G / N} \hat{f}_{A} d \nu_{2}$. In fact, for any finite collection $\left\{B_{1}, \ldots, B_{n}\right\}$ of disjoint subsets of $G$,

$$
\sum_{i=1}^{n} c_{i} \chi_{B_{i}} \leq \hat{f}_{A}\left(g^{-1} \cdot\right) \text { if and only if } \sum_{i=1}^{n} c_{i} \chi_{g \cdot B_{i}} \leq \hat{f}_{A}(\cdot)
$$


So, since the integrals are approximated by simple functions, we have

$$
\begin{aligned}
\int_{G / N} \sum_{i=1}^{n} c_{i} \chi_{B_{i}} d \nu_{2} & =\sum_{i=1}^{n} c_{i} \int_{G / N} \chi_{B_{i}} d \nu_{2} \\
& =\sum_{i=1}^{n} c_{i} \nu_{2}\left(B_{i}\right)=\sum_{i=1}^{n} c_{i} \nu_{2}\left(g \cdot B_{i}\right) \\
& =\int_{G / N} \sum_{i=1}^{n} c_{i} \chi_{g \cdot B_{i}} d \nu_{2},
\end{aligned}
$$

and we can conclude that:

$$
\int_{G / N} \hat{f}_{A}\left(g^{-1} \cdot t\right) d \nu_{2}=\int_{G / N} \hat{f}_{A}(t) d \nu_{2}
$$

Then, $G$ is amenable.

Proposition 1.3.3. If $G$ is a direct union of a directed system of amenable groups $\left\{G_{\alpha}\right\}_{\alpha \in I}$ then $G$ is amenable.

Proof. Assume that $G=\bigcup_{\alpha \in I} G_{\alpha}$, where each $G_{\alpha}$ is amenable witnessed by the measure $\mu_{\alpha}$. Consider the topological space $[0,1]^{\mathcal{P}(G)}$ with the product topology, and let $\mathcal{M}_{\alpha}$ be the collection of all functions $\mu \in[0,1]^{\mathcal{P}(G)}$ such that $\mu$ is a finitely additive measure on $G, \mu(G)=1$ and $\mu(g \cdot A)=\mu(A)$ for all $g \in G_{\alpha}$.

- Claim 1: $\mathcal{M}_{\alpha}$ is not empty

Define $\mu: \mathcal{P}(G) \rightarrow[0,1]$ as $\mu(A)=\mu_{\alpha}\left(A \cap G_{\alpha}\right)$. Notice that $\mu(\emptyset)=\mu_{\alpha}(\emptyset)=0$ and $\mu(G)=\mu_{\alpha}\left(G \cap G_{\alpha}\right)=\mu_{\alpha}\left(G_{\alpha}\right)=1$. Also, if $A, B \subseteq G$ are disjoint, then

$$
\begin{aligned}
\mu(A \cup B) & =\mu\left((A \cup B) \cap G_{\alpha}\right)=\mu_{\alpha}\left(\left(A \cap G_{\alpha}\right) \cup\left(B \cap G_{\alpha}\right)\right) \\
& =\mu_{\alpha}\left(A \cap G_{\alpha}\right)+\mu_{\alpha}\left(B \cap G_{\alpha}\right)=\mu(A)+\mu(B) .
\end{aligned}
$$


Finally, let $g \in G_{\alpha}$ and $A \subseteq G$. Then

$$
\mu(g \cdot A)=\mu_{\alpha}\left(g \cdot A \cap G_{\alpha}\right)=\mu_{\alpha}\left(g \cdot\left(A \cap G_{\alpha}\right)\right)=\mu_{\alpha}\left(A \cap G_{\alpha}\right)=\mu(A) .
$$

Therefore, $\mu \in \mathcal{M}_{\alpha}$.

Claim 1

- Claim 2: $\mathcal{M}_{\alpha}$ is closed

We will show that $\mathcal{M}_{\alpha}^{c}$ is open. Suppose that $f \notin \mathcal{M}_{\alpha}$. Then either $f(\emptyset) \neq 0$, or $f(G) \neq 1$, or $f(A) \neq f(g \cdot A)$ for some $g \in G_{\alpha}$ and $A \subseteq G$, or $f(A \cup B) \neq f(A)+f(B)$ for some disjoint subsets $A, B$ of $G$. Let us write $f(A)=f_{A} \in[0,1]$ for $A \subseteq G$. We can consider the four cases separately:

- If $f_{\emptyset} \neq 0$, consider the set $V=V^{0}=\prod_{X \in \mathcal{P}(G)} V_{X}^{0}$ where $V_{\emptyset}^{0}=(0,1]$ and $V_{X}^{0}=[0,1]$ for $X \neq \emptyset$.

- If $f_{G} \neq 1$, take $V=V^{1}=\prod_{X \in \mathcal{P}(G)} V_{X}^{1}$ where $V_{1, G}=[0,1)$ and $V_{X}^{1}=[0,1]$ for $X \neq G$

- If $f_{g \cdot A} \neq f_{A}$ for some $g \in G_{\alpha}$ and $A \subseteq G$, we may assume without loss of generality that $f_{g \cdot A}<r<f_{A}$ for some $r \in[0,1]$ and take $V=V^{g, A}=\prod_{X \in \mathcal{P}(G)} V_{X}^{g, A}$ where $V_{g \cdot A}^{g, A}=[0, r), V_{A}^{g, A}=(r, 1]$ and $V_{1, X}=[0,1]$ for $X \neq g \cdot A, A$. Notice that if $f^{\prime} \in V$, then $f_{A}^{\prime}<r<f_{g \cdot A}^{\prime}$.

- If $f_{A}+f_{B} \neq f_{A \cup B}$ for a $A, B \subseteq G$ disjoint, we can take

$$
\epsilon=\frac{\left|f_{A}+f_{B}-f_{A \cup B}\right|}{4}>0
$$

and consider the set $V=V^{A, B}=\prod_{X \in \mathcal{P}(G)} V_{X}^{A, B}$ where $V_{A}^{A, B}=\left(f_{A}-\epsilon, f_{A}+\epsilon\right), V_{B}^{A, B}=$ $\left(f_{B}-\epsilon, f_{B}+\epsilon\right), V_{A \cup B}^{A, B}=\left(f_{A \cup B}-\epsilon, f_{A \cup B}+\epsilon\right)$ and $V_{X}^{A, B}=[0,1]$ for $X \neq A, B, A \cup B$ 
Notice that if $f^{\prime} \in V$, then

$$
\begin{aligned}
\left|f_{A}^{\prime}+f_{B}^{\prime}-f_{A \cup B}^{\prime}\right| & =\left|f_{A \cup B}^{\prime}-f_{A}^{\prime}-f_{B}^{\prime}\right| \\
& =\left|\left(f_{A}+f_{B}-f_{A \cup B}\right)-\left(f_{A}^{\prime}-f_{A}+f_{B}^{\prime}-f_{B}-f_{A \cup B}^{\prime}-f_{A \cup B}\right)\right| \\
& \geq\left|\left(f_{A}+f_{B}-f_{A \cup B}\right)\right|-\left|f_{A}^{\prime}-f_{A}+f_{B}^{\prime}-f_{B}-f_{A \cup B}^{\prime}-f_{A \cup B}\right| \\
& \geq\left|\left(f_{A}+f_{B}-f_{A \cup B}\right)\right|-\left|f_{A}^{\prime}-f_{A}\right|-\left|f_{B}^{\prime}-f_{B}\right|-\left|f_{A \cup B}^{\prime}-f_{A \cup B}\right| \\
& \geq 4 \epsilon-\epsilon-\epsilon-\epsilon=\epsilon>0 .
\end{aligned}
$$

and so, $f_{A}^{\prime}+f_{B}^{\prime} \neq f_{A \cup B}^{\prime}$.

In any case, we have that $V$ is an basic open $[0,1]^{\mathcal{P}(G)}$ with the product topology, $f \in V$, and $V \subseteq \mathcal{M}_{\alpha}^{c}$ because given $f^{\prime} \in V$, one of the conditions that define $\mathcal{M}_{\alpha}$ fails for $f^{\prime}$. Therefore, we can conclude that $\mathcal{M}_{\alpha}$ is closed.

- Claim 3: $\left\{\mathcal{M}_{\alpha}\right\}_{\alpha \in I}$ has the finite intersection property.

Since $G$ is the direct limit of a directed system, given $\alpha_{1}, \ldots, \alpha_{n} \in I$ there is $\gamma \in I$ such that $G_{\gamma}$ embeds the groups $G_{\alpha_{1}}, \ldots, G_{\alpha_{n}}$. We may assume without loss of generality that each $G_{\alpha_{i}}$ is a subgroup of $G_{\gamma}$. Notice that if a probability measure that is $G_{\gamma}$-leftinvariant, then it is $G_{\alpha_{i}}$-left-invariant for every $i=1, \ldots, n$. Thus, $\bigcap_{i=1}^{n} \mathcal{M}_{\alpha_{i}} \supseteq M_{\gamma} \neq \emptyset$ by Claim 1.

$\square_{\text {Claim 3. }}$

Now we can show that $G$ is amenable. Since $[0,1]^{\mathcal{P}(G)}$ is compact (by Tychonoff's theorem) and $\left\{\mathcal{M}_{\alpha}\right\}_{\alpha \in I}$ is a family of closed sets with the finite intersection property, then $\bigcap_{\alpha \in I} \mathcal{M}_{\alpha} \neq \emptyset$. Let $\mu \in \bigcap_{\alpha \in I} \mathcal{M}_{\alpha}$, it is clear that $\mu$ is a finitely additive measure and that $\mu(G)=1$ (since $\mu \in \mathcal{M}_{\alpha}$ ), so it remains to show that $\mu$ is $G$-invariant. Let $g \in G$ and $A \subseteq G$, then there is an $\alpha \in I$ such that $g \in G_{\alpha}$. Hence, since $\mu \in \mathcal{M}_{\alpha}, \mu(A)=\mu(g \cdot A)$. We conclude that $G$ is amenable.

Lemma 1.3.4. Every group is the direct limit of their finitely generated subgroups.

Proof. Consider $\mathcal{I}=\{i \subset G \mid i$ is a finitely generated subgroup of $G\}$. We will say that 
$i \leq j$ if and only if $i$ is a subgroup of $j$, and the homomorphism $f_{i j}: i \rightarrow j$ are the inclusion maps. Firstly, we will show that $\left(\mathcal{I},\left\{f_{i j}\right\}_{i j}\right)$ is a directed system:

- Let $i, j, k \in \mathcal{I}$ such that $i \leq j \leq k$, then $f_{i j}\left(f_{j k}(x)\right)=f_{i j}(x)=x=f_{i k}(x)$ and $f_{i i}$ is the identity of $i$.

- Let $i, j \in \mathcal{I}$ and let $\left\{g_{1}, \cdots, g_{m}\right\}$ and $\left\{g_{1}^{\prime}, \cdots, g_{l}^{\prime}\right\}$ be their respective generators. Let $k$ be the group generated by $\left\{g_{1}, \cdots, g_{m}, g_{1}^{\prime}, \cdots, g_{l}^{\prime}\right\}$. Clearly $i$ and $j$ are subgroups of $k$.

We can define $\mathcal{H}=\lim _{\rightarrow} i=\bigsqcup i / \sim$, where for $x_{i} \in i, x_{j} \in j$, we say that $\left(x_{i}, i\right) \sim\left(x_{j}, j\right)$ if and only if $\exists k \in \mathcal{I}$ such that $f_{i k}\left(x_{i}\right)=f_{j k}\left(x_{j}\right)$ (they are related if eventually there are the same element in the directed system). And let $\phi_{i}: i \rightarrow \mathcal{H}$ defined as $\phi_{i}(x)=[(x, i)]_{\sim}$. Now consider the inclusion maps $\psi_{i}: i \rightarrow G$, and notice that $\forall i, j \in \mathcal{I}, \phi_{j} \circ f_{i j}=\phi_{i}$. Therefore, by the universal property of $\mathcal{H}$ there is an homomorphism $u: \mathcal{H} \rightarrow G$, such that for all $i \in \mathcal{I}, u \circ \phi_{i}=\psi_{i}$, and since $\psi$ is injective $u$ is an embedding. Observe that $u$ is also surjective, because for some $g \in G$ we can consider $i=\langle g\rangle$, and since $u \circ \phi_{i}=\psi_{i}$, then $g=\phi_{i}(g)=u \circ \phi_{i}(g)=u\left([(g, i)]_{\sim}\right)$.

Theorem 1.3.5. Every abelian group is amenable.

Proof. We start by showing that every finitely generated abelian group is amenable. Let $\epsilon>0$ and $G$ an abelian group generated by $\left\{g_{1}, \ldots, g_{m}\right\}$. If $\mu \in[0,1]^{\mathcal{P}(G)}$ we will say that $\mu$ is an $\epsilon$-invariant if:

- $\mu(G)=1$

- $\mu$ is a finitely additive measure,

- For all $A \subseteq G$ and $g_{i}$ a generator, then $\left|\mu(A)-\mu\left(g_{i} \cdot A\right)\right| \leq \epsilon$

Define $\mathcal{M}_{\epsilon}=\left\{\mu \in[0,1]^{\mathcal{P}(G)} \mid \mu\right.$ is an $\epsilon$ - invariant measure $\}$. Firstly, we will show that $\mathcal{M}_{\epsilon} \neq \emptyset$.In order to clarify the idea we will assume that $G$ is generated by one element $g$. 
Let $N \in \mathbb{N}$ be such that $\frac{2}{N}<\epsilon$ and define $\mu_{\epsilon}: \mathcal{P}(G) \rightarrow[0,1]$ as $\mu_{\epsilon}(A)=\frac{\left|\left\{i \in \mathbb{N} \mid 0 \leq i \leq N g^{i} \in A\right\}\right|}{N}$. Notice that $\mu_{\epsilon}(G)=\frac{N}{N}=1$, because $g^{i} \in G$ for $1 \leq i \leq N$. Also, $\mu_{\epsilon}(\emptyset)=0$. Consider now $A, B$ disjoint subsets of $X$. If $g^{i} \in A$ for some $0 \leq i \leq N$ then $g^{i} \notin B$. Therefore $\mu(A \cup B)=\mu(A)+\mu(B)$.

Finally, notice that $|\mu(A)-\mu(g \cdot A)| \leq \frac{|\{1, N\}|}{N}=\frac{2}{N} \leq \epsilon$, since for all $1 \leq i<N$ we have that $g^{i} \in A$ if and only if $g^{i+1} \in g \cdot A$ (but it might happen that $g \in g \cdot A$ or $g^{N} \in A$ ).

It is possible to show that $\mathcal{M}_{\epsilon}$ is a closed subset of $[0,1]^{\mathcal{P}(G)}$ in a similar way as in the proof of Proposition 1.3.3. Now, consider the family $\mathcal{F}=\left\{\mathcal{M}_{\epsilon}\right\}_{\epsilon>0}$, and observe that $\mathcal{F}$ has the finite intersection property. In fact, for $\epsilon_{1}, \cdots, \epsilon_{n}$ we can take $\epsilon=\min \left\{\epsilon_{i} \mid 1 \leq i \leq n\right\}$ and $\emptyset \neq \mathcal{M}_{\epsilon} \subseteq \bigcap_{i=1}^{n} \mathcal{M}_{\epsilon_{i}}$. Therefore, since $[0,1]^{\mathcal{P}(G)}$ is compact there is an element $\mu \in \bigcap_{\epsilon>0} \mathcal{M}_{\epsilon}$, which will be a finitely additive measure satisfying $\mu(G)=1$ and for every $A \subset G, \mu(A)=\mu(g \cdot A)$. So, for every $A \subseteq G$, and $h=g^{n} \in G$, we have $\mu(A)=\mu(g \cdot A)=\cdots=\mu(\underbrace{g \cdots g}_{n \text {-times }} A)=\mu(h \cdot A)$. We conclude that $G$ is invariant.

If $G$ has $\left\{g_{1}, \cdots, g_{m}\right\}$ generators, let $N$ be such that $\frac{2}{N^{m}}<\epsilon$ and define

$$
\mu_{\epsilon}(A)=\frac{\left|\left(i_{1}, \cdots, i_{m}\right)\right| 1 \leq i_{1}, \cdots, i_{m} \leq N g_{1}^{i_{1}} \cdots g_{m}^{i_{m}} \in A \mid}{N^{m}}
$$

Note that we need $G$ to be abelian in order to show that $\mu_{\epsilon}$ is well-defined (one can write the finite products of generators as $\left.g_{1}^{i_{1}} \cdots g_{m}^{i_{m}}\right)$ and to show $\epsilon$-invariance of $\mu_{\epsilon}$.

Now, if $G$ is an abelian group, then every finitely generated subgroup of $G$ is also abelian and thus amenable by the first part of this proof. By Lemma 1.3.4, $G$ is a direct limit of its finitely generated subgroups, and since they are all amenable, by Proposition 1.3.3, we conclude that $G$ is amenable.

Definition 1.3.6 (Abelian-by-finite). Let $(G, \cdot, e)$ be a group, we say that $G$ is abelianby-finite if there is a normal abelian subgroup $N$ of $G$ such that $[G: N]$ is finite.

Corollary 1.3.7. Every group which is abelian-by-finite is amenable. 
Proof. Let $G$ be a group and suppose that there is a normal subgroup such that $N$ is abelian and its index in $G$ is finite. Then by Theorem 1.3.5, $N$ is amenable and by the Lemma 1.3.2 (1), $G / N$ is amenable. Finally, by Lemma 1.3.2 (4) $G$ is amenable. 


\section{Chapter 2}

\section{Analysis of definable groups in Presburger Arithmetic}

The main goal of this thesis is to understand the definable groups in Presburger Arith-

metic. In particular, we are interested in finding a characterization of $G / G^{00}$ where $G$ is a Presburger-definable group. In order to reach a good understanding of the definable groups we recall a few important results that simplify the description of definable sets, such as quantifier elimination and cell decomposition theorem. The first one is a folklore result which can be found in [12], while the latter one is a theorem proved by R. Cluckers in [3].

Since these results will be very useful to characterize definable sets, we decided to present a detailed exposition of the ideas developed by Cluckers and Marker, for a sake of completeness. We found new proofs for some of the technical lemmas.

In the second part of this section we presented two notions of dimension for the Presburger definable sets, similar to the o-minimal context: one comes from the cell decomposition theorem and the second arises from definable closure. Cluckers introduced these two notions in [3] and he proved they coincide. We presented here a detailed proof 
of this fact, which will allow us to extend some useful tools from the o-minimal setting to the Presburger setting, these facts are known as folklore.

Through this chapter $T$ will denote the theory of the structure $(\mathbb{Z},<,+, 0,1)$ and $\mathcal{M}$ will be a very large saturated model of $T$, and $G$ will always denote a definable group in Presburger Arithmetic.

\subsection{Presburger Language and quantifier elimination}

Proposition 2.1.1. The theory $T=\operatorname{Th}(\mathbb{Z},+,<, 0,1)$ does not eliminate quantifiers in the language $\mathcal{L}^{-}=\{+,<,-, 0,1\}$

Proof. Define the formulas $E(x)=\exists z(z+z=x)$ and $O(x)=\exists z(z+z+1=x)$, and consider the two-type $\Gamma\left(y_{1}, y_{2}\right)$ given by

$$
\Gamma\left(y_{1}, y_{2}\right)=\left\{E\left(y_{1}\right), O\left(y_{2}\right)\right\} \cup\{y_{1}, y_{2}>\underbrace{1+\cdots+1}_{n \text {-times }}: n<\omega\} .
$$

Clearly $\Gamma\left(y_{1}, y_{2}\right)$ is finitely satisfiable, and by compactness, there are elements $b_{1}, b_{2} \in \mathcal{M}$ satisfying $\Gamma\left(y_{1}, y_{2}\right)$. Notice that $\operatorname{tp}_{\mathcal{L}^{-}}\left(b_{1}\right) \neq \operatorname{tp}_{\mathcal{L}^{-}}\left(b_{2}\right)$ because $\mathcal{M} \models E\left(b_{1}\right) \wedge O\left(b_{2}\right) \wedge$ $\forall x(E(x) \leftrightarrow \neg O(x))$.

Let $M_{1}$ and $M_{2}$ be the $\mathcal{L}^{-}$-substructures of $\mathcal{M}$ generated by $b_{1}$ and $b_{2}$, respectively. Notice that $M_{i}$ is the subgroup of $\mathcal{M}$ generated by $1, b_{i}$, for $i=1,2$. Thus, we can take the map $f: M_{1} \rightarrow M_{2}$ given by $f\left(m+n b_{1}\right)=m+n b_{2}$ for any $m, n \in \mathbb{Z}$. This will define an isomorphism between $M_{1}$ and $M_{2}$, and since elementary maps preserve quantifierfree formulas, we conclude that $\operatorname{qftp}_{\mathcal{L}^{-}}\left(b_{1}\right)=\operatorname{qftp}_{\mathcal{L}^{-}}\left(b_{2}\right)$. This shows that Presburger arithmetic does not have quantifier elimination in the language $\{+,<, 0,1\}$.

Remark 2.1.2. In fact, in the proof the previous proposition, it can be shown that $M_{1}$ is isomorphic to the structure $Z^{2}=\left\langle\mathbb{Z} \oplus \mathbb{Z},+,<_{\text {lex }}, \mathbf{0}^{Z^{2}}=(0,0), \mathbf{1}^{Z^{2}}=(0,1)\right\rangle$ where $<_{\text {lex }}$ 
is the lexicographical order in $\mathbb{Z} \oplus \mathbb{Z}$, since we can identify $b_{1}$ with $(1,0)$.

Consider the language $\mathcal{L}_{\text {Pres }}=\left\{+,-,<,\left\{\equiv_{n}\right\}_{n \in \mathbb{N}}, 0,1\right\}$, where $\equiv_{n}$ is a binary relation defined as $x \equiv_{n} y:=\exists z(\underbrace{z+\cdots+z}_{n-\text { times }}=x-y)$.

The theory $\operatorname{Th}(\mathbb{Z},+,<, 0,1)$ has quantifier elimination in the language $\mathcal{L}_{\text {Press. }}$. In fact, the theory $\operatorname{Pr}$ for Presburger arithmetic can be axiomatized by the following sentences:

(i) Axioms for ordered abelian groups.

(ii) $0<1$.

(iii) $\forall x(x \leq 0 \wedge x \geq 1)$.

(iv) $)_{n} \forall x\left(x \equiv_{n} y \leftrightarrow \exists z(x-y=\underbrace{z+\cdots+z}_{n \text {-times }})\right.$ for $n=2,3, \ldots$

$(\mathrm{v})_{n} \forall x\left(\bigvee_{i=0}^{n-1}(x \equiv_{n} \underbrace{1+\cdots+1}_{i \text {-times }} \wedge \bigwedge_{j \neq i} \neg x \equiv_{n} \underbrace{1+\cdots+1}_{j \text {-times }})\right)$.

We refer the reader to [12] for more details.

\subsubsection{Some consequences of quantifier elimination}

The following statements are immediate consequences from quantifier elimination, and will be very important for understanding the decomposition of definable sets into cells presented by Cluckers in [3]. We believe that these results are folklore, but nevertheless we proof then ourselves.

\subsubsection{A-definable functions and $d c l(A)$}

Let $A$ be a small set of parameters of $\mathcal{M}$. In this section we want to use quantifier elimination to find and explicit characterization of the complete 1-types over A. Moreover, we use this theorem to describe an element $b \in \operatorname{dcl}(A)$ as the image of an $A$ - linear function. 
Definition 2.1.3 (A-linear function). Let $f: X \subseteq \mathcal{M}^{m} \rightarrow \mathcal{M}$ be a function. We say that $f$ is $A$-linear, if it can be written in the form

$$
f(\bar{x})=\sum_{i=1}^{m} s_{i}\left(\frac{x_{i}-c_{i}}{k_{i}}\right)+\gamma
$$

where $\gamma \in d c l(A)$, and for each $1 \leq i \leq m, 0 \leq c_{i}<k_{i}$ and $s$ are integers, such that $x_{i} \equiv_{k_{i}} c_{i}$.

Fact 2.1.4. Let $x$ be a single variable and $\bar{a}=\left(a_{1}, \ldots, a_{k}\right)$ be a tuple of elements in $\mathcal{M}$. Then, the terms $\tau(x, \bar{a})$ are of the form:

$$
s x+\sum_{i=1}^{n} k_{i} a_{i}+n
$$

where $s_{i}, k_{i}, n$ are integers.

Proof. We proceed by induction on the length of $\tau(x, \bar{a})$. Note that the terms depending on $\bar{a}$ of length 1 are $0,1, x, a_{1}, \ldots, a_{k}$, which are of the desired form.

Induction Hypothesis: Let $\sigma(x, \bar{a})$ be a term of lenght less than $n$. Then, $\sigma(x, \bar{a})$ is a $\mathbb{Z}$-linear combination of $x$ and $1, a_{1}, \ldots, a_{k}$.

Let now $\tau(x, \bar{a})$ be a term of length $n$. Assume that $\tau(x, \bar{a})=\sigma_{1}(x, \bar{a})+\sigma_{2}(x, \bar{a})$. By the induction hypothesis:

$$
\tau(x, \bar{a})=\left(s^{1} x+\sum_{i=1}^{n} k_{i}^{1} a_{i}+n^{1}\right)+\left(s^{2} x+\sum_{i=1}^{n} k_{i}^{2} a_{i}+n^{2}\right)
$$

Then,

$$
\tau(x, \bar{a})=\underbrace{\left(s^{1}+s^{2}\right)}_{\in \mathbb{Z}} x+\sum_{i=1}^{n} \underbrace{\left(k_{i}^{1}+k_{i}^{2}\right)}_{\in \mathbb{Z}} a_{i}+\underbrace{\left(n^{1}+n^{2}\right)}_{\in \mathbb{Z}}
$$

If $\tau(x, \bar{a})=\sigma_{1}(x, \bar{a})-\sigma_{2}(x, \bar{a})$ the result follows in a similar way. 
Lemma 2.1.5. Let $\sigma(x, \bar{a})$ be an atomic consistent $\mathcal{L}_{\text {Pres }}$-formula, with parameters. Then $\sigma(x, \bar{a})$ is equivalent to a formula of one of the following forms:

1. $x=b$, where $b \in \operatorname{dcl}(\bar{a})$.

2. $x \leq b$, where $b \in \operatorname{dcl}(\bar{a})$.

3. $x \geq b$, where $b \in \operatorname{dcl}(\bar{a})$.

4. $x \equiv_{N} c$, where $0 \leq c<N$ are integers.

Proof. Let $\sigma(x, \bar{a})$ be an atomic formula. Then it has one of the following forms:

Remark 2.1.6. In all the following proofs we will assume that the coefficients $s_{1}$ and $s_{2}$ are different, because otherwise we can reduce the formula to something equivalent avoiding to use the variable $x$. (Example: $2+x=1+1+x$ is equivalent to $2=1+1$.)

Case 1: $\tau_{1}(x, \bar{a})=\tau_{2}(x, \bar{a})$. By Fact 2.1.4, this equality is an equation of the form:

$$
s^{1} x+\sum_{i=1}^{n} k_{i}^{1} a_{i}+l^{1}=s^{2} x+\sum_{i=1}^{n} k_{i}^{2} a_{i}+l^{2} .
$$

Then, we can rewrite this equation so that the coefficient of $x$ remains not negative:

$$
\left(s^{1}-s^{2}\right) x=\sum_{i=1}^{n}\left(k_{i}^{2}-k_{i}^{1}\right) a_{i}+\left(l^{2}-l^{1}\right)
$$

Let $m=s^{1}-s^{2}, k_{i}=k_{i}^{2}-k_{i}^{1}$ and $l^{3}=l^{2}-l^{1}$. For each $a_{i}$ we can choose some $0 \leq c_{i}<m$ such that $a_{i}-c_{i} \equiv_{m} 0$, hence:

$$
m x=\sum_{i=1}^{n} k_{i}\left(a_{i}-c_{i}\right)+\sum_{i=1}^{n} k_{i} c_{i}+l^{3} .
$$


Letting $z=\sum_{i=1}^{n} k_{i} c_{i}+l^{3}$, we have that $z \equiv_{m} 0$, since:

$$
z=m x-\sum_{i=1}^{n} k_{i}\left(a_{i}-c_{i}\right) \equiv_{m} 0
$$

Therefore,

$$
x=\sum_{i=1}^{n}\left(\frac{k_{i}\left(a_{i}-c_{i}\right)}{m}\right)+\gamma
$$

and, since $\sum_{i=1}^{n}\left(\frac{k_{i}\left(a_{i}-c_{i}\right)}{m}\right)+\gamma \in \operatorname{dcl}(\bar{a})$ we have the desired result.

Case 2: $\tau_{1}(x, \bar{a})<\tau_{2}(x, \bar{a})$. Again, by Fact 2.1.4, we may assume that this formula is:

$$
s^{1} x+\sum_{i=1}^{n} k_{i}^{1} a_{i}+n^{1}<s^{2} x+\sum_{i=1}^{n} k_{i}^{2} a_{i}+n^{2}
$$

Then, we can rewrite the equation $m$ the coefficient of $x$ is positive, and we have that:

$$
m x<\sum_{i=1}^{n}\left(k_{i}^{2}-k_{i}^{1}\right) a_{i}+\left(n^{2}-n^{1}\right)
$$

Hence, letting $k_{i}=k_{i}^{2}-k_{i}^{1}$ and taking $0 \leq c_{i}<m$ such that $a_{i} \equiv_{m} c_{i}$ we have that:

$$
m x<\sum_{i=1}^{n} k_{i}\left(a_{i}-c_{i}\right)+\sum_{i=1}^{n} k_{i} c_{i}+\left(n^{2}-n^{1}\right)
$$

so, if we define $z=\sum_{i=1}^{n} k_{i} c_{i}+\left(n^{2}-n^{1}\right)$ we can choose an element $0 \leq c<m$ such that $z \equiv_{m} c$. Since $c<m$, the set defined by $\sigma(x, \bar{a})$, is the same set defined by:

$$
m x \leq \sum_{i=1}^{n} k_{i}\left(a_{i}-c_{i}\right)+(z-c)
$$


Then, we conclude it is also equivalent to:

$$
m x \leq \sum_{i=1}^{n} k_{i}\left(\frac{a_{i}-c_{i}}{m}\right)+\left(\frac{z-c}{m}\right) \in \operatorname{dcl}(\bar{a})
$$

Case 3: $\tau_{1}(x, \bar{a})>\tau_{2}(x, \bar{a})$, it is similar to the previous case.

Case 4: $\tau_{1}(x, \bar{a}) \equiv_{k} \tau_{2}(x, \bar{a})$, then by Fact 2.1.4 this formula is of the form:

$$
s^{1} x+\sum_{i=1}^{n} k_{i}^{1} a_{i}+n^{1} \equiv_{k} s^{2} x+\sum_{i=1}^{n} k_{i}^{2} a_{i}+n^{2}
$$

Again, we can rewrite the equation so that $m$ is a positive integer, then we have that:

$$
m x \equiv_{k} \sum_{i=1}^{n} k_{i} a_{i}+n
$$

By the axiom $(v)_{n}$, there is a unique $0 \leq c<k$ such that $\sum_{i=1}^{n} k_{i} a_{i}+n \equiv_{k} c$. Then the set defined by $\sigma(x, \bar{a})$ is equivalent to

$$
m x \equiv_{k} c
$$

and this equation can be reduced to a unique system $x \equiv_{l} d$, where $0 \leq d<l$ are integers.

Fact 2.1.7. Let $\sigma(x, \bar{a})$ be an atomic formula. Then $\neg(\sigma(x, \bar{a}))$ is equivalent to a disjunction of positive atomic formulas.

Proof. By Lemma 2.1.5, we may assume that $\sigma(x, \bar{a})$ has one of the following forms:

Case 1: $x=b$, where $b \in d c l(\bar{a})$. In this case, $\neg(\sigma(x, \bar{a}))$ is equivalent to $\neg(x=b)$. Since $<$ is a linear order, $\neg(x=b)$ is equivalent to $(x<b) \vee(x>b)$, so we are done. 
Case 2: $x \leq b$, where $b \in \operatorname{dcl}(\bar{a})$. In this case, $\neg(\sigma(x, \bar{a}))$ is equivalent to $\neg(x \leq b)$. Since $<$ is a linear order, $\neg(x \leq b)$ is equivalent to $(x>b)$, so we are done.

Case 3: $x \geq b$, where $b \in \operatorname{dcl}(\bar{a})$. In this case, $\neg(\sigma(x, \bar{a}))$ is equivalent to $\neg(x \geq b)$. Since $<$ is a linear order, $\neg(x \geq b)$ is equivalent to $(x<b)$, so we are done.

Case 4: $x \equiv_{N} c$, where $0 \leq c<N$ are integers. Therefore, $\neg(\sigma(x, \bar{a}))$ is equivalent to $\neg\left(x \equiv_{N} c\right)$. By the axiom $(v)_{n}, \neg\left(x \equiv_{N} c\right)$ is equivalent to:

$$
\left(x \equiv_{N} 0\right) \vee\left(x \equiv_{N} 1\right) \vee \cdots \vee\left(x \equiv_{N} c-1\right) \vee\left(x \equiv_{N} c+1\right) \vee \cdots \vee\left(x \equiv_{N} N-1\right)
$$

Corollary 2.1.8. Let $A$ be a set of parameters. Then $b \in \operatorname{dcl}(A)$ if and only if there is a definable linear function $\alpha$ and a tuple $\bar{a} \subseteq A$ such that $\alpha(\bar{a})=b$.

Proof. The right-to-left direction follows by the definition of definable closure. For the converse, let $b \in \operatorname{dcl}(A)$, then there is an $\mathcal{L}(A)$ formula $\phi(x, \bar{a})$ such that $b$ is the only element in $\mathcal{M}$ such that $\mathcal{M} \models \phi(v ; \bar{a})$. By quantifier elimination, $\phi(x, \bar{a})$ is equivalent to a boolean combination of atomic formulas

$$
\bigvee_{i=1}^{k}\left(\bigwedge_{j=1}^{p} \sigma_{j}^{i}\left(x, a_{i j}\right)\right)
$$

Since the negation of an atomic formula is equivalent to a disjunction of positive atomic formulas, we may assume that every formula $\sigma_{j}^{i}\left(x, a_{i j}\right)$ is positive (Since the formula that we obtain replacing each negative formula by a disjunction of positive atomic formulas can be replaced by its disjunctive normal form.)

Then, there is some $i \leq k$, such that $\mathcal{M} \models \bigwedge_{j=1}^{p} \sigma_{j}^{i}\left(b, a_{i j}\right)$, where $a_{i j}$ are terms depending on $\bar{a}$. 
Assume first that for some $j \leq p$ we have $\sigma_{j}(x ; \bar{a}):=x=t(\bar{a})$. Since every $\mathcal{L}_{\text {Press }}$-term defines an $A$-linear function, we can take $\alpha$ to be the $A$-linear function given by $t(\bar{y})$, and we are done.

Thus, we may assume that for each $j$, either $\sigma_{j}^{i}\left(x, a_{i j}\right):=x<d_{j}$, or $\sigma_{j}^{i}\left(x, a_{i j}\right)=x>d_{j}$, or $\sigma_{j}^{i}\left(x, a_{i j}\right)=\left(x \equiv_{N_{j}} d_{j}\right)$, and in every case $d_{j}$ is a term depending only on $\bar{a}$.

Take $d=\max \left\{d_{j} \mid \mathcal{M} \models d_{j}<b\right\}$ and $d^{\prime}=\min \left\{d_{j} \mid \mathcal{M} \models b<d_{j}\right\}$. Observe that if the interval $\left(d, d^{\prime}\right)$ is infinite, then by the axioms of Presburger there would be infinitely many elements satisfying $\phi(x ; \bar{a})$. Thus, the interval $\left(d, d^{\prime}\right)$ is finite, and there is some $k \leq d^{\prime}-d \in \mathbb{N}$ such that $b=d+k$. Since $d=t(\bar{a})$ for some term in $\mathcal{L}_{\text {Pres }}$, the function $\alpha(\bar{x})=t(\bar{x})+k$ is an $A$-linear function such that $\alpha(\bar{x})=b$.

Definition 2.1.9 $(d c l(A)$-cut). Let $A$ be a small set of parameters. A $d c l(A)$ - cut is a consistent and complete set of formulas of the form $d_{1}<x<d_{2}$, where $d_{1}, d_{2} \in d c l(A)$.

Fact 2.1.10 (1-types on Presburger). Let $A$ be a small set set of parameters. Let $q(x)$ a complete 1-type over $A$, then this type has one of the following forms:

1. There is an element $d$ such that $x=d$, where $d \in d c l(A)$.

2. For every element $d \in d c l(A)$ we have $x \neq d \in q(x)$, and then $(x<d) \in q(x)$ or $(x>d) \in q(x)$. Therefore, $q(x)$ is completely determined by a dcl $(A)$ - cut and a set of formulas of the form $x \equiv_{n} c_{n}, 0 \leq c_{0}<n$ for each $n \in \mathbb{N}$.

Proof. Let $\phi(x, \bar{a}) \in q(x)$, by and the reduction of the negation of an atomic formula to a conjunction of positive atomic formulas, this formula is equivalent to a formula of the form

$$
\bigvee_{i=1}^{k}\left(\bigwedge_{j=1}^{p} \sigma_{j}^{i}\left(x, a_{i j}\right)\right)
$$


where $\sigma_{j}^{i}\left(x, a_{i j}\right)$ is a positive atomic $A$-formula. Since $q(x)$ is complete, we have

$$
\bigwedge_{j=1}^{p}\left(\left(\sigma_{j}^{i}\left(x, a_{i j}\right)\right) \in q(x)\right.
$$

for some $i \leq k$, and by Lemma 2.1.5 we have the desired result.

\subsection{Cell Descomposition theorem}

Definition 2.2.1 (A-definable cells). We will define the $A$-definable cells inductively as follows:

1. A A-definable 0-cell is a point $p \in d c l(A)$. An $A$-definable 1 -cell is an infinite set of the form:

$\left\{\alpha \square_{1} x \square_{2} \beta \mid x \equiv_{N} c\right\}$, where $\alpha, \beta \in d c l(A), 0 \leq c<N$ are positive integers and $\square_{j}$ stands for either $\leq$ or no condition condition.

2. Assume that we have defined a $\left(i_{1}, \ldots, i_{n}\right) A$-definable cell $C$, where $i_{j} \in\{0,1\}$ for all $j \leq n$. Then:

- An A-definable $\left(i_{1}, \ldots, i_{n}, 0\right)$-cell, is a set $\left\{(\bar{x}, t) \in \mathcal{M}^{n+1} \mid \bar{x} \in C \wedge t=\alpha(\bar{x})\right\}$ where $\alpha$ is an $A$-definable function.

- An A-definable $\left(i_{1}, \ldots, i_{n}, 1\right)$-cell is a set of the form:

$$
D=\left\{(\bar{x}, t) \in \mathcal{M}^{n+1} \mid \bar{x} \in C \wedge\left(\left(\alpha(\bar{x}) \square_{1} t \square_{2} \beta(\bar{x})\right) \mid t \equiv_{N} k\right)\right\}
$$

where $\alpha, \beta$ are $A$-definable functions defined on $C, 0 \leq k<N$ are positive integers, the symbol $\square_{i}$ represents either $\leq$ or no condition for $i=1,2$ and the size of the fibers $F_{\bar{x}}=\{t \in \mathcal{M} \mid(\bar{x}, t) \in D\}$ cannot be bounded uniformly over $C$, meaning that there is no $N \in \mathbb{N}$ such that for all $\bar{x} \in C,\left|F_{\bar{x}}\right| \leq N$.

Example 2.2.2. The following are examples of definable cells over some set of parameters. 
- Let $n \in \mathbb{Z}$, the point $\{n\}$ is a $\emptyset$-definable set.

- Let $\alpha$ be a non-standard element, such that for every element $n \in \mathbb{Z}$, we have that $n<\alpha$. Then, the set:

$$
D=\left\{x \in \mathcal{M} \mid 0 \leq x \leq \alpha \wedge x \equiv_{2} 1\right\}
$$

is a $1 \alpha$ - definable cell.

- Let $\beta(x)=3\left(\frac{x-1}{2}\right)+2$ then the set $\left\{(x, t) \in \mathcal{M}^{2} \mid x \in D \wedge t=\beta(x)\right\}$ is a $(1,0) \alpha$ definable cell.

- Let $\gamma(x)=x+\alpha$, then the set:

$$
C=\left\{(x, t) \mid x \in D \wedge \beta(x) \leq t \leq \gamma(x) \wedge t \equiv_{3} 0\right\}
$$

is a $(1,1) \alpha$-definable cells. (Note that the fiber $(\beta(1), \gamma(1))$ is infinite, since $\beta(1)=2$ and $\gamma(1)=\alpha+1$.)

Definition 2.2.3 (Partition into cells ). Let $X$ be a definable set. A partition $\mathcal{P}$ of $X$ over $A$ is a finite set $\left\{C_{1}, \ldots, C_{n}\right\}$ of pairwise disjoint $A$-definable cells such that $X=\bigcup_{i=1}^{n} C_{i}$.

Definition 2.2.4 (Piecewise $A$-linear function). Let $X$ be a definable set and $f: X \rightarrow \mathcal{M}$ a definable function. We say that $f$ is a piecewise $A$-linear function, if there is a partition $\mathcal{P}$ of $X$ into $A$-definable cells such that, $f \uparrow_{C}: C \rightarrow \mathcal{M}$ is an $A$-linear function.

The following theorem, proved by R. Cluckers in [3], provides a characterization of the definable sets in Presburger Arithmetic in terms of definable cells, and is a fundamental tool for our analysis of definable groups in Presburger Arithmetic. Moreover, it also allows us to assume that every definable function is piecewise linear.

Theorem 2.2.5. [Cell Decomposition Theorem] 
1. Let $X$ be an $A$-definable set. Then there is a partition $\mathcal{P}$ of $X$ in A-definable cells.

2. Let $X \subseteq \mathcal{M}^{n}$ and $f: X \rightarrow \mathcal{M}$ be definable. Then there is a finite partition $\mathcal{P}$ of $X$ in cells such that $f \uparrow_{C}: C \rightarrow \mathcal{M}$ is linear for each cell $C \in \mathcal{P}$. Moreover, if $X$ and $f$ are A-definable, then we can take the cells also A-definable.

Proof. see [3], Theorem 1.

Lemma 2.2.6. Let $\mathcal{P}_{1}$ and $\mathcal{P}_{2}$ be two cell-decompositions of an A-definable set $X$. Then, there is a partition $\mathcal{P}$ of $X$ in $A$-definable cells, such that for every element $C \in \mathcal{P}$ and $A \in \mathcal{P}_{i}$, either $C \subseteq A$ or $C \cap A=\emptyset$. This partition $\mathcal{P}$ is called a refinement of $\mathcal{P}_{1}$ and $\mathcal{P}_{2}$.

Proof. Notice that it is enough to show that the union of two $n$ - cells $C_{1}, C_{2}$ there is a partition $\mathcal{Q}$ of $C_{1} \cup C_{2}$ such that for every $D \in \mathcal{Q}$ we have that $D \subseteq C_{i}$ or $D \cap C_{i}=\emptyset$ for $i=1,2$. Since $\mathcal{P}_{1} \cup \mathcal{P}_{2}$ is finite, if we refine every union of two cells, at the end we can take the union of all those refinement and we have the desired partition.

We proceed by induction on $n$, where $X \subseteq \mathcal{M}^{n}$.

Base Case: $n=1$, Case 1: $C_{1}$ and $C_{2}$ are 1-cells. Assume that $C_{1}=\left\{\alpha_{1} \square_{1}^{1} x \square_{1}^{2} \beta_{1} \wedge\right.$ $\left.x \equiv_{N_{1}} c_{1}\right\}$ and $C_{2}=\left\{\alpha_{2} \square_{2}^{1} x \square_{2}^{2} \beta_{2} \wedge x \equiv_{N_{2}} c_{2}\right\}$. Without loss of generality we can assume that $\square_{i}^{j}=\leq$, and $\alpha_{1}<\alpha_{2}<\beta_{1}<\beta_{2}$. If $\left(\alpha_{2}, \beta_{1}\right)$ is finite, then for each element $x$ in this interval there is a $j \in \mathbb{N}$ such that $x=\alpha_{2}+j$, and so $\left\{\alpha_{2} \leq x \leq \beta_{1} \mid x \equiv_{N_{1}} c_{1} \wedge x \equiv_{N_{2}}\right.$ $\left.c_{2}\right\}=\bigcup_{i=1}^{k}\left\{\alpha_{2}+j_{i}\right\}$. Thus, we can define the following partition of $C_{1} \cap C_{2}$ as:

$$
C_{1} \cap C_{2}=\bigcup_{i=1}^{k}\left\{\alpha_{2}+j_{i}\right\}
$$

Since each point $\alpha_{2}+j_{i}$ is a 0 - cell then we can write:

$$
C_{1} \cup C_{2}=\left\{\alpha_{1} \leq x \leq \alpha_{2} \mid x \equiv_{N_{1}} c_{1}\right\} \cup \bigcup_{i=1}^{k}\left\{\alpha_{2}+j_{i}\right\} \cup\left\{\beta_{1} \leq x \leq \beta_{2} \mid x \equiv_{N_{2}} c_{2}\right\}
$$


and we are done. Now assume that that $\left(\alpha_{2}, \beta_{1}\right)$ is infinite. Then, we know that the linear system of congruences $x \equiv_{N_{1}} c_{1}$ and $x \equiv_{N_{2}} c_{2}$ can be reduced to a unique system of the form $x \equiv_{N} c$, therefore:

$$
C_{1} \cap C_{2}=\left\{\alpha_{2} \leq x \leq \alpha_{1} \mid x \equiv_{N} c\right\}
$$

However, we need to shatter the interval $\left(\alpha_{2}, \beta_{1}\right)$ in all the possible combinations of the linear system congruences. Consider the following systems:

$$
\begin{aligned}
& x \equiv_{N_{1}} c_{1} \wedge x \equiv_{N_{2}} 0, x \equiv_{N_{1}} c_{1} \wedge x \equiv_{N_{2}} 1, \ldots, x \equiv_{N_{1}} c_{1} \wedge x \equiv_{N_{2}} N_{2}-1 \\
& x \equiv_{N_{2}} c_{2} \wedge x \equiv_{N_{1}} 0, x \equiv_{N_{2}} c_{2} \wedge x \equiv_{N_{1}} 1, \ldots, x \equiv_{N_{2}} c_{2} \wedge x \equiv_{N_{2}} N_{1}-1 .
\end{aligned}
$$

We know that all these $k$ - systems can be reduced to a unique system of the form, $x \equiv_{N_{k}} c_{k}$. Therefore, we can consider the partition of $C_{1} \cup C_{2}$ as:

$C_{1} \cup C_{2}=\left\{\alpha_{1} \leq x \leq \alpha_{2} \mid x \equiv_{N_{1}} c_{1}\right\} \cup \bigcup_{j=1}^{k}\left\{\alpha_{2} \leq x \leq \beta_{1} \mid x \equiv_{N_{k}} c_{k}\right\} \cup\left\{\beta_{1} \leq x \leq \beta_{2} \mid x \equiv_{N_{2}} c_{2}\right\}$

Case 2: $C_{1}$ is a 1-cell and $C_{2}$ is a 0 -cell

Assume that $C_{2}=\{p\}$ and $C_{1}=\left\{\alpha \leq x \leq \beta \mid x \equiv_{N} c\right\}$, if $p \notin C_{1}$, then we are done. Assume that $p \in C_{1}$, then $C_{1} \cap C_{2}=\{p\}$ which is a 0 -cell. Since $(\alpha, \beta)$ is infinite, then $(\alpha, p)$ or $(\beta, p)$ is infinite. Without loss of generality, assume that $(\alpha, p)$ is finite and $(p, \beta)$ is infinite. Then, if $(\alpha, p)$ is finite, then for each element $x \in[\alpha, p]$ we can find some $j \in \mathbb{N}$ such that $x=\alpha+j$, therefore the set:

$$
\left\{\alpha \leq x \leq p \mid x \equiv_{N} c\right\}=\bigcup_{i=1}^{k}\{\alpha+j\}
$$


then we have that:

$$
C_{1} \cup C_{2}=\bigcup_{i=1}^{k}\{\alpha+j\} \cup\left\{p \leq x \leq \beta \mid x \equiv_{N} c\right\} .
$$

Case 3: $C_{1}$ and $C_{2}$ are both 0 -cells

Then, $C_{1}$ and $C_{2}$ are already disjoint or $C_{1}=C_{2}=\{p\}$. So we are done.

\section{Inductive step:}

Induction Hypothesis: Assume that for every definable pair of cells $Y_{1}, Y_{2} \subset \mathcal{M}^{n}$, then there is a partition $\mathcal{Q}$ of $Y_{1} \cup Y_{2}$ in cells such that for every $D \in \mathcal{Q}$ we have that $D \subseteq Y_{i}$ or $D \cap Y_{i}=\emptyset$ for $i=1,2$.

Let $X_{1}$ and $X_{2}$ be two $n+1$-cells, then consider:

$$
\begin{aligned}
& \pi\left(X_{1}\right)=\left\{\bar{x} \in \mathcal{M}^{n} \mid \exists t(\bar{x}, t) \in X_{1}\right\}, \\
& \pi\left(X_{2}\right)=\left\{\bar{x} \in \mathcal{M}^{n} \mid \exists t(\bar{x}, t) \in X_{2}\right\} .
\end{aligned}
$$

Since $Y_{1}=\pi\left(X_{1}\right)$ and $Y_{2}=\pi\left(X_{2}\right)$ are $n$-cells, then by the inductive hypothesis there is a cell decomposition $\mathcal{Q}$ of $Y_{1} \cup Y_{2}$ such that for every $D \in \mathcal{Q}$, we have that $D \subseteq Y_{i}$ or $D \cap Y_{i}=\emptyset$ for $i=1,2$.

Fix a cell $C$ in the decomposition. Without loss of generality assume that $X_{1}$ and $X_{2}$ are $\left(i_{1}, \ldots, i_{n}, 1\right)$ cells, since the other cases follow similarly. Then $X_{1}$ and $X_{2}$ are of the following form:

$$
\begin{aligned}
& X_{1}=\left\{(\bar{x}, t)\left|\bar{x} \in Y_{1}\right| \alpha_{1}(\bar{x}) \leq t \leq \beta_{1}(\bar{x}) \wedge t \equiv_{N_{1}} c_{1}\right\} \\
& X_{2}=\left\{(\bar{x}, t)\left|\bar{x} \in Y_{2}\right| \alpha_{2}(\bar{x}) \leq t \leq \beta_{2}(\bar{x}) \wedge t \equiv_{N_{2}} c_{2}\right\}
\end{aligned}
$$


Then, we can shatter $C$ into a finite union disjoint sets such that on each of these sets the functions $\alpha_{1}(\bar{x}), \alpha_{2}(\bar{x}), \beta_{1}(\bar{x}), \beta_{2}(\bar{x})$ are ordered. (In fact this statement can be said in our language, for example consider $x \in C \wedge \alpha_{1}(\bar{x}) \leq \beta_{1}(x) \leq \alpha_{2}(\bar{x}) \leq \beta_{2}(\bar{x})$. By cell decomposition each of these sets is a finite union of definable cells.) Let $A$ be one of those cells, and without loss of generality assume that the functions on $A$ are ordered as follow:

$$
\alpha_{1}(\bar{x}) \leq \alpha_{2}(\bar{x}) \leq \beta_{1}(\bar{x}) \leq \beta_{2}(\bar{x})
$$

If $A_{\bar{x}}=\left\{\alpha_{1}(\bar{x}) \leq t \leq \alpha_{2}(\bar{x}) \mid t \equiv_{N_{1}} c_{1}\right\}$ is uniformly bounded by $k \in \mathbb{N}$. We can also shatter $A$ as a finite union of disjoint definable sets $B_{0}, \ldots, B_{N_{1}-1}$, where each $B_{i}=\{\bar{x} \in$ $\left.A \mid \alpha_{1}(\bar{x}) \equiv_{N_{1}} i\right\}$. Each of these sets is definable, then there is a cell decomposition $\mathcal{B}_{i}$ of $B_{i}$ for each $i$. Fix $F$ one of those cells, and without loss of generality assume that it is contained in $B_{0}$. (In particular $\alpha(\bar{x}) \equiv_{N_{1}} 0$, which implies that the first element in the fiber $A_{\bar{x}}$ is $\alpha(\bar{x})+c_{1}$.)

Now, we can defined the following subsets of $F$,

$$
F_{1}=\left\{\bar{x} \in F:\left|A_{\bar{x}}\right| \geq 1\right\}, F_{2}=\left\{\bar{x} \in F:\left|A_{\bar{x}}\right| \geq 2\right\}, \ldots, F_{k}=\left\{\bar{x} \in F:\left|A_{\bar{x}}\right|=k\right\}
$$

and notice that each $F_{i}$ is definable and $F_{k} \subseteq F_{k-1} \subseteq \cdots \subseteq F_{2} \subseteq F_{1}$. Also, we can define the following linear functions:

$$
\begin{gathered}
f_{1}:=F_{1} \rightarrow \mathcal{M} \\
f_{1}(\bar{x})=\alpha_{1}(\bar{x})+c_{1},
\end{gathered}
$$




$$
\begin{aligned}
f_{k} & :=F_{k} \rightarrow \mathcal{M} \\
f_{k}(\bar{x}) & =\alpha_{1}(\bar{x})+(k-1) N_{1}+c_{1} .
\end{aligned}
$$

Now consider the following definable subsets of $F$,

$$
G_{1}=\left\{\bar{x} \in F|| A_{\bar{x}} \mid=1\right\}, \ldots, G_{k}=\left\{\bar{x} \in F|| A_{\bar{x}} \mid=k\right\}
$$

Each of those sets is definable and moreover they are disjoint. Therefore, there is a cell decomposition $\mathcal{R}_{i}$ of $G_{i}$. Take one those cells, name it $R$ and then consider the following union of cells:

$$
\bigcup_{j=1}^{i}\left\{(\bar{x}, t) \in \mathcal{M}^{n+1} \mid \bar{x} \in R \wedge t=f_{j}(\bar{x})\right\} .
$$

Doing this process for each cell in $\mathcal{B}_{0}$ we obtain a partition of $\left\{(\bar{x}, t) \mid \bar{x} \in B_{0} \wedge \alpha_{1}(\bar{x}) \leq\right.$ $\left.t \leq \alpha_{2}(\bar{x})\right\}$. Since the cell $A$ is the disjoint union of $B_{0}, \ldots, B_{N-1}$, doing this for each of the sets $B_{i}$ we obtain a partition of the set $\left\{(\bar{x}, t) \mid \bar{x} \in A \wedge \alpha_{1}(\bar{x}) \leq t \leq \alpha_{2}(\bar{x})\right\}$.

On the other hand, if the fibers $A_{\bar{x}}=\left\{\alpha_{1}(\bar{x}) \leq t \leq \alpha_{2}(\bar{x}) \mid t \equiv_{N_{1}} c_{1}\right\}$ cannot be uniformly bounded, then we define

$$
F=\left\{(\bar{x}, t) \mid \bar{x} \in A \wedge \alpha_{1}(\bar{x}) \leq t \leq \alpha_{2}(\bar{x}) \wedge t \equiv_{N_{1}} c_{1}\right\}
$$

Doing this for each interval of functions, $\left(\alpha_{1}(\bar{x}), \alpha_{2}(\bar{x})\right),\left(\alpha_{2}(\bar{x}), \beta_{1}(\bar{x})\right)$ and $\left(\beta_{1}(\bar{x}), \beta_{2}(\bar{x})\right)$ and taking the partition desired following the cases above ( whether the fibers are uniformly bounded or not), we obtain a complete decomposition of the set:

$$
\left\{(\bar{x}, t) \in X_{1} \cup X_{2} \mid \bar{x} \in A\right\}
$$


Repeating this process for each cell $A$ we obtain a partition of $\left\{(\bar{x}, t) \in X_{1} \cup X_{2} \mid \bar{x} \in C\right\}$, since the partition of $Y_{1} \cup Y_{2}$ is finite, following this argument for each cell $C$ in the cell decomposition of $Y_{1} \cup Y_{2}$ we obtain the desired partition.

Remark 2.2.7. Remember that in the interval $\left(\alpha_{2}(\bar{x}), \beta_{1}(\bar{x})\right)$ we have to shatter each congruence into all the possible combinations, and all these systems can be reduced to a unique system of the form $x \equiv_{N_{k}} c_{k}$, as we did in the base case.

\subsection{Dimension}

In this section we introduce the two notions of dimension presented by R. Cluckers in [3]. However, our presentation includes a detailed proof of the fact that these two notions coincide.We also present several results that can be obtained from the dimension.

\subsubsection{Definable Dimension}

Let $\mathcal{M}$ be a saturated model of Presburger Arithmetic. Notice that since $\mathcal{M}$ is an ordered structure, then the algebraic closure coincides with the definable closure.

Definition 2.3.1 (Definable dimension).

1. Let $\bar{a}=\left(a_{1}, \ldots, a_{n}\right) \in M^{n}$ and $A$ a set of parameters. The dimension of $(\bar{a} / A)$ is the size of a maximal independent subset of $\left\{a_{1}, \ldots, a_{n}\right\}$ with respect to the definable closure. Namely, $\operatorname{dim}(\bar{a})=k$ if there are $a_{i_{1}}, \ldots, a_{i_{k}} \in\left\{a_{1}, \ldots, a_{n}\right\}$ such that:

- $a_{i_{1}} \notin d c l(A)$, and $a_{i_{j+1}} \notin d c l\left(a_{i_{1}}, \ldots, a_{i_{j}}, A\right)$ for $j=1,2, \ldots, k$.

- $a_{j} \in d c l\left(a_{i_{1}}, \ldots, a_{i_{k}}, A\right)$ for all $j=1, \ldots, n$.

2. Let $X \subseteq \mathcal{M}^{n}$ be an $A$-definable set. We define the dimension of $X$ by:

$$
\operatorname{dim}_{d e f}(X)=\max \{\operatorname{dim}(\bar{a} / A): \bar{a} \in X\}
$$


In order to prove that this notion of dimension is well defined we need to show that the theory of Presburger Arithmetic has the exchange property. This was a result proved by O. Belegradek, Y. Peterzil and F. Wagner in [2].

Definition 2.3.2 (Exchange Property). We say that Presburger Arithmetic has the exchange property if for every pair of elements $a, b \in \mathcal{M}$ and $C$ a set of parameters, we have that if $b \in \operatorname{dcl}(a C) \backslash d \operatorname{dcl}(C)$ then $a \in \operatorname{dcl}(b C)$.

Lemma 2.3.3. Presburger Arithmetic has the exchange property.

Proof. Let $a, b \in \mathcal{M}$ and $C$ a set of parameters. Assume that $b \in \operatorname{dcl}(a C)$, then by Corollary 2.1.8, there is a linear function $\alpha$ and a tuple $\left(c_{1}, \ldots, c_{n}\right)=\bar{c} \subseteq C$, such that $b=\alpha(a \bar{c})$.Without loss of generality, we may assume that $\alpha$ has the following form:

$$
b=s_{1}\left(\frac{a-d_{1}}{n_{1}}\right)+\sum_{i=1}^{k} s_{1+i}\left(\frac{c_{i}-d_{1+i}}{n_{1+i}}\right)+l
$$

where for each $i \leq k+1, s_{i}$ and $0 \leq c_{i}<n_{i}$ and $l$ are integers.

Therefore,

$$
s_{1}\left(\frac{a-d_{1}}{n_{1}}\right)=\underbrace{b-\sum_{i=1}^{k} s_{1+i}\left(\frac{c_{i}-d_{1+i}}{n_{1+i}}\right)-l}_{S}
$$

Since $b \notin d c l(C), s_{1}$ should be different than 0 . And notice that $S \equiv_{s_{1}} 0$, then

$$
a-d_{1}=n_{1}\left(\frac{b-\sum_{i=1}^{k} s_{1+i}\left(\frac{c_{i}-d_{1+i}}{n_{1+i}}\right)-l}{s_{1}}\right)
$$

Therefore,

$$
a=n_{1}\left(\frac{b-\sum_{i=1}^{k} s_{1+i}\left(\frac{c_{i}-d_{1+i}}{n_{1+i}}\right)-l}{s_{1}}\right)+d_{1}
$$


So we conclude that $a \in d c l(C b)$, as we desired.

Corollary 2.3.4. For any definable set $X$ in Presburger, $\operatorname{dim}_{\text {def }}(X)$ is well defined.

Definition 2.3.5. Let $X \subset \mathcal{M}^{k}$ be a definable set in Presburger Arithmetic. We say that $\bar{a} \in X$ is dim-generic if $\operatorname{dim}(\bar{a})=\operatorname{dim}(X)$.

Lemma 2.3.6. Let $X, Y \subseteq \mathcal{M}^{n}$ be two definable sets. Then

$$
\operatorname{dim}_{d e f}(X \cup Y)=\max \left\{\operatorname{dim}_{d e f}(X), \operatorname{dim}_{d e f}(Y)\right\}
$$

Proof. Assume that $X$ and $Y$ are definable over $A$. Note that

$$
\begin{aligned}
\operatorname{dim}_{\text {def }}(X \cup Y) & =\max \{\operatorname{dim}(\bar{y} / A): \bar{y} \in X \cup Y\} \\
& =\max \{\max \{\operatorname{dim}(\bar{y} / A): \bar{y} \in X\}, \max \{\operatorname{dim}(\bar{y} / A): \bar{y} \in Y\}\} \\
& =\max \left\{\operatorname{dim}_{\text {def }}(X), \operatorname{dim}_{\text {def }}(Y)\right\}
\end{aligned}
$$

Lemma 2.3.7. Let $\bar{a}, \bar{b}$ be two tuples of elements in $\mathcal{M}$, and $A \subseteq \mathcal{M}$. Then

$$
\operatorname{dim}(\bar{a}, \bar{b} / A)=\operatorname{dim}(\bar{a} / A)+\operatorname{dim}(\bar{b} / A \bar{a})
$$

Proof. Let $\left\{a_{i_{1}}, \ldots, a_{i_{k}}\right\}$ be a basis for $\bar{a}$ over $A$ (i.e., a maximal independent subset of $\bar{a}$ ), and take $b_{j_{1}}, \ldots, b_{j_{\ell}}$ be a basis of $\bar{b}$ over $A \bar{a}$. Then $\operatorname{dim}(\bar{a} / A)=k$ and $\operatorname{dim}(\bar{b} / A \bar{a})=\ell$. To prove the equality above it is enough to show that the set $S=\left\{a_{i_{1}}, \ldots, a_{i_{k}}, b_{j_{1}}, \ldots, b_{j_{\ell}}\right\}$ is a basis for $\bar{a}, \bar{b}$ over $A$.

Independence follows directly from the construction, and maximality is clear: if $x \in \bar{a} \bar{b}$ then either $x \in \bar{a}$ and $x \in \operatorname{dcl}\left(a_{i_{1}}, \ldots, a_{i_{k}}, A\right)$, or $x \in \bar{b}$ and $x \in \operatorname{dcl}\left(b_{i_{1}}, \ldots, b_{j_{\ell}}, A, a_{i_{1}}, \ldots, a_{i_{k}}\right)$.

We conclude then that $S$ is a basis of $\bar{a}, \bar{b}$ over $A$, thus by definition of dimension we have $\operatorname{dim}(\bar{a}, \bar{b} / A)=|S|=k+\ell=\operatorname{dim}(\bar{a} / A)+\operatorname{dim}(\bar{b} / A \bar{a})$.

Lemma 2.3.8. Let $X \subseteq \mathcal{M}^{n}$ be an $A$-definable set and $f: X \rightarrow \mathcal{M}^{m}$ be an $A$ definable function. Then, $\operatorname{dim}_{\text {def }}(f(X)) \leq \operatorname{dim}_{\text {def }}(X)$. Moreover, if $f$ is injective, then 
$\operatorname{dim}_{d e f}(f(X))=\operatorname{dim}_{\text {def }}(X)$.

Proof. Suppose $\bar{a} \in X$ and $f(\bar{b}) \in f(X)$ are chosen so that $\operatorname{dim}(X)=\operatorname{dim}(\bar{a} / A)$ and $\operatorname{dim}_{d e f}(f(X))=\operatorname{dim}_{d e f}(f(\bar{b}) / A)$. Notice that every element in the tuple $f(\bar{b})$ can be obtained definably from $\bar{b}$ and $A$ by composing with the projection maps. So, $\operatorname{dim}_{\operatorname{def}}(f(\bar{b}) / A \bar{b})=$ 0 and by Lemma 2.3.7 we obtain:

$$
\begin{aligned}
\operatorname{dim}_{\text {def }}(f(X)) & =\operatorname{dim}(f(\bar{b}) / A) \leq \operatorname{dim}(\bar{b}, f(\bar{b}) / A) \\
& =\operatorname{dim}(\bar{b} / A)+\operatorname{dim}(f(\bar{b}) / A \bar{b})=\operatorname{dim}(\bar{b} / A) \\
& \leq \operatorname{dim}(\bar{a} / A)=\operatorname{dim}_{\text {def }}(X) .
\end{aligned}
$$

Suppose now that $f$ is a bijection. Note that for every $\bar{b} \in X$, we have $f(\bar{b}) \in d c l(A, \bar{b})$ and $\bar{b} \in \operatorname{dcl}(A, f(\bar{b}))$, and so $\operatorname{dim}(f(\bar{b}) / A, \bar{b})=0=\operatorname{dim}(\bar{b} / A, f(\bar{b}))$. Thus, by Lemma 2.3.7 we have

$$
\begin{aligned}
\operatorname{dim}(\bar{b} / A) & =\operatorname{dim}(\bar{b} / A)+\operatorname{dim}(f(\bar{b}) / A, \bar{b}) \\
& =\operatorname{dim}(\bar{b}, f(\bar{b}) / A) \\
& =\operatorname{dim}(f(\bar{b}) / A)+\operatorname{dim}(\bar{b} / A, f(\bar{b})) \\
& =\operatorname{dim}(f(\bar{b}) / A)
\end{aligned}
$$

In particular, $\operatorname{dim}(f(X))=\max \{\operatorname{dim}(f(\bar{b}) / A): \bar{b} \in X\}=\max \{\operatorname{dim}(\bar{b} / A): \bar{b} \in X\}=$ $\operatorname{dim}(X)$.

\subsubsection{Cell Dimension}

Definition 2.3.9 (Cell dimension). Let $C$ be an $A$-definable $\left(i_{1}, \ldots, i_{n}\right)$-cell. We define its cell-dimension as $\operatorname{dim}_{\text {cell }}(C)=\sum_{j=1}^{n} i_{j}$.

Lemma 2.3.10. Let $C$ be an A-definable $\left(i_{1}, \ldots, i_{n}\right)$-cell. Then $\operatorname{dim}_{\text {cell }}(C)=\operatorname{dim}_{\text {def }}(C)$. 
Proof. We proceed by induction on $n$.

- Case $n=1$ : We consider two cases.

If $C$ is a 0 -cell, then $C=\{p\}$ for some $p \in \operatorname{dcl}(A)$, and we obtain $\operatorname{dim}_{\text {def }}(p)=0=$ $i_{1}=\operatorname{dim}_{\text {cell }}(C)$. If $C$ is a 1 -cell, then there are $\alpha, \beta \in d c l(A)$ and $0 \leq c<N$ positive integers such that $C$ is an infinite set defined by the formula $\left(\alpha \square_{1} x \square_{2} \beta\right) \wedge x \equiv_{N} c$. Let us assume that $\square_{1}$ and $\square_{2}$ are both $\leq$. The other cases follow similarly.

Consider the type $p(x)=\{x \in C\} \cup\{x \neq d \mid d \in \operatorname{dcl}(A) \cap C\}$. We now show using compactness that this type is consistent: let $\Sigma_{0}(x)=\{x \in C\} \cup\left\{x \neq d_{i}\right.$ : $i=1, \ldots, n\}$ be a finite subset of $p(x)$, where $d_{1}, \ldots, d_{n} \in \operatorname{dcl}(A) \cap C$. Since $d_{1}, \ldots, d_{k} \in C$, then we have $\alpha \leq d_{1}, \ldots, d_{n} \leq \beta$. Without loss of generality, we may furthermore assume that $d_{1}<d_{2}<\cdots<d_{n}$. Put $d_{0}:=\alpha$ and $d_{n+1}:=\beta$.

Since $C$ is an infinite set, then at least one of the intervals $\left[d_{i}, d_{i+1}\right]($ for $0 \leq i \leq n)$ is infinite. Thus, if $\left[d_{i}, d_{i+1}\right]$ is infinite, then $d_{i}+j \equiv_{N} c$, for some $j \leq N$, and the element $x_{0}=d_{i}+j$ realizes $\sigma_{0}$. We conclude that $p(x)$ is finitely consistent, and by compactness, $p(x)$ is consistent.

Since $\mathcal{M}$ is saturated, there is an element $b \in \mathcal{M}$ realizing the type $p(x)$. We then have by construction that $\operatorname{dim}_{\text {cell }}(C)=1=\operatorname{dim}(b / A)=\operatorname{dim}_{d e f}(C)$.

- Inductive step: We consider again two cases.

Suppose that $C$ is an $A$-definable $\left(i_{1}, \ddot{i}_{n}, 0\right)$ cell, say defined by $C=\{(\bar{x}, t): \bar{x} \in$ $D$ and $\alpha(\bar{x})=t\}$ for an $A$-definable $\left(i_{1}, \ldots, i_{n}\right)$-cell $D$ and an $A$-definable function $\alpha: D \rightarrow M$. Furthermore, by Theorem 2.2.5, we may assume (by considering a more refined partition) that $f$ is an $A$-linear function.

By induction hypothesis, $\sum_{j=1}^{n} i_{j}=\operatorname{dim}_{\text {cell }}(D)=\operatorname{dim}_{d e f}(D)$. Notice also that for every $\bar{d} \in D$ we have that $\alpha(\bar{d})$ is $A$-definable over $A \bar{d}$, and so $\operatorname{dim}(\alpha(\bar{d}) / A \bar{d})=0$. 
Thus,

$$
\begin{aligned}
\operatorname{dim}_{\text {def }}(C) & =\max \{\operatorname{dim}((\bar{d}, \alpha(\bar{d})) / A: \bar{d} \in D\} \\
& =\max \{\operatorname{dim}(\bar{d} / A)+\operatorname{dim}(\alpha(\bar{d}) / A): d \in D\} \\
& =\max \{\operatorname{dim}(\bar{d} / A): \bar{d} \in D\} \\
& =\operatorname{dim}_{\text {def }}(D)=\operatorname{dim}_{\text {cell }}(D) \\
& =\sum_{j=1}^{n} i_{j}+0=\operatorname{dim}_{\text {cell }}(C)
\end{aligned}
$$

Suppose now that $C$ is $\mathrm{n} A$-definable $\left(i_{1}, \ldots, i_{n}, 1\right)$-cell, defined by

$$
C=\left\{(\bar{x}, t) \mid \bar{x} \in D \wedge\left(\left(\alpha(\bar{x}) \square_{1} t \square_{2} \beta(\bar{x})\right) \wedge t \equiv_{N} c\right)\right\}
$$

where $D$ is an $A$-definable $\left(i_{1}, \ldots, i_{n}\right)$-cell, $0 \leq c<N$ are positive integers and $\alpha, \beta$ are chosen to be $A$-definable linear functions. As in the proof of case $n=1$, let us assume that $\square_{1}$ and $\square_{2}$ are both $\leq$. The other cases follow in a similar way.

The functions $A$-definable linear functions $\alpha, \beta$ defined on $D$, have the forms

$$
\alpha(\bar{x})=\sum_{i=1}^{n} s_{i}\left(\frac{x_{i}-d_{i}}{m_{i}}\right)+\gamma_{1}, \quad \beta(\bar{x})=\sum_{i=1}^{n} p_{i}\left(\frac{x_{i}-e_{i}}{k_{i}}\right)+\gamma_{2},
$$

By the inductive hypothesis, $\sum_{j=1}^{n} i_{j}=\operatorname{dim}_{\text {cell }}(D)=\operatorname{dim}_{\text {def }}(D)$. Consider a tuple $\bar{b} \in D$, such that $\operatorname{dim}(\bar{b} / A)=\operatorname{dim}(D)$, and consider a subtuple $\left\{b_{i_{1}}, \ldots, b_{i_{k}}\right\}$ of $\bar{b}$ that is an algebraically independent set over $A$.

Also, the set $I=\left\{t \in \mathcal{M} \mid \alpha(\bar{b}) \leq t \leq \beta(\bar{b}) \wedge t \equiv_{N} c\right\}$ is infinite. Suppose for a contradiction that $I$ is finite, then there is some $j \in \mathbb{N}$ such that $\beta(\bar{b})=\alpha(\bar{b})+j$. 
So we have

$$
\sum_{i=1}^{n} p_{i}\left(\frac{b_{i}-e_{i}}{k_{i}}\right)+\gamma_{2}=\sum_{i=1}^{n} s_{i}\left(\frac{b_{i}-d_{i}}{m_{i}}\right)+\gamma_{1}+j
$$

Note that for all $j \notin\left\{i_{1}, \ldots, i_{k}\right\}$ there is an $A$-definable function $\delta_{j}$ such that $\delta_{j}\left(b_{i_{1}}, \ldots, b_{i_{k}}\right)=b_{j}$. Then replacing all these equations in (1) (for $\left.b_{j}\right)$ we can isolate $b_{i_{k}}$, concluding that $b_{i_{k}} \in d c l\left(A b_{i_{1}}, \ldots, b_{i_{k-1}}\right)$. This contradicts the fact that $b_{i_{1}}, \ldots, b_{i_{k}}$ are algebraically independent over $A$.

Notice that for every $\bar{d} \in D,(\alpha(\bar{d}), \beta(\bar{d}))$ is an $A \bar{d}$-definable cell of dimension at most 1 . So, since the set $I=[\alpha(\bar{b}), \beta(\bar{b})] \subseteq \mathcal{M}$ is infinite, it defines a $A \bar{b}$-definable 1-cell and we have

$$
\begin{aligned}
\operatorname{dim}_{\text {def }}(C) & =\max \{\operatorname{dim}((\bar{d}, t) / A):(\bar{d}, t) \in C\}, \\
& =\max \{\operatorname{dim}(\bar{d} / A)+\operatorname{dim}(t / A \bar{d}):(\bar{d}, t) \in C\}, \quad \text { (by Lemma 2.3.7) } \\
& =\operatorname{dim}(\bar{b} / A)+1=\operatorname{dim}_{\text {def }}(D)+1, \\
& =\left(\sum_{j=1}^{n} i_{j}\right)+1=\operatorname{dim}_{\text {cell }}(C) .
\end{aligned}
$$

Corollary 2.3.11. Let $X \subseteq \mathcal{M}^{n}$ be an A-definable set. For any partition $\mathcal{P}$ of $X$ into A-definable cells we have:

$$
\begin{aligned}
\operatorname{dim}_{\text {def }}(X) & =\max \left\{i_{1}+\cdots+i_{n} \mid C \in \mathcal{P}, C \text { is a }\left(i_{1}, \ldots, i_{n}\right) \text {-cell }\right\} \\
& =\max \left\{i_{1}+\cdots+i_{n} \mid C \text { is a }\left(i_{1}, \ldots, i_{n}\right) \text {-cell contained in } X\right\} .
\end{aligned}
$$

Proof. We will show the first equality, $\operatorname{dim}_{\text {def }}(X)=\max \left\{\operatorname{dim}_{\text {def }}(C) \mid C \in \mathcal{P}\right\}$. Since, for 
any definable $A$-cell, $\operatorname{dim}_{\text {cell }}(C)=\operatorname{dim}_{\text {def }}(C)$, and $X=\bigcup_{i=1}^{k} C_{i}$, then we have:

$$
\begin{aligned}
\operatorname{dim}_{\text {def }}(X) & =\max \left\{\operatorname{dim}_{\text {def }}(C) \mid C \in \mathcal{P}\right\} \\
& =\max \left\{\operatorname{dim}_{\text {cell }}(C) \mid C \in \mathcal{P}\right\} \\
& =\max \left\{i_{1}+\cdots+i_{n} \mid C \in \mathcal{P} \text { and } C \text { in an }\left(i_{1}, \ldots, i_{n}\right)-\text { cell }\right\}
\end{aligned}
$$

For the second equality, let $C$ be an $\left(i_{1}, \ldots, i_{n}\right)$ a cell contained in $X$, then:

$$
i_{1}+\cdots+i_{n}=\operatorname{dim}_{\text {cell }}(C)=\operatorname{dim}_{\text {def }}(C) \leq \operatorname{dim}_{\text {def }}(X)
$$

Thus, $\max \left\{i_{1}+\cdots+i_{n} \mid C\right.$ is a cell contained in $\left.X\right\} \leq \operatorname{dim}_{\text {def }}(X)$.

Conversely, let $\bar{b} \in X$ be a tuple such that $\operatorname{dim}_{\text {def }}(X)=\operatorname{dim}(\bar{b} / A)$. By cell decomposition there is a partition $\mathcal{Q}$ of $X$ into definable cells. Then, there is a cell $C \in \mathcal{Q}$, such that $\bar{b} \in C$, and thus

$$
\begin{aligned}
\operatorname{dim}_{\text {def }}(X) & =\operatorname{dim}(\bar{b} / A) \leq \operatorname{dim}_{\text {def }}(C)=\operatorname{dim}_{\text {cell }}(C) \\
& \leq \max \left\{i_{1}+\cdots+i_{n} \mid C \text { is a cell contained in } X\right\}
\end{aligned}
$$

This last corollary implies that the two notions of dimension given actually coincide. Therefore, from now on we will simply write $\operatorname{dim}(X)$. One of the main applications of the notion of dimension in the o-minimal context is that it provides a way to distinguish the subsets of a given set $X$ that are particularly large. Namely, we say that $Y \subseteq X$ is a "big" subset of $X$ when $\operatorname{dim}(Y)=\operatorname{dim}(X)$. This analogy, used in the context of Presburger Arithmetic, will allow us to characterize whether a subgroup $H$ of a definable group $G$ is large, and this can be done in two ways: using dimensions or using the index of $H$ in $G$.

Lemma 2.3.12. Let $G$ be a definable group in $\mathcal{M}$. Let $H$ be a definable subgroup of $G$. Then $\operatorname{dim}(H)=\operatorname{dim}(G)$ if and only if $H$ has finite index in $G$. 
Proof. $(\Leftarrow)$ If $H$ has finite index in $G$, then $G=a_{1} \cdot H \cup \cdots \cup a_{k} \cdot H$, for some ${ }_{1}, \ldots, a_{k} \in G$. By Lemma 2.3 .8 we have $\operatorname{dim}\left(a_{i} H\right)=\operatorname{dim}(H)$ for each $i \leq k$. Thus, $\operatorname{dim}(G)=\max \left\{\operatorname{dim}\left(a_{i} \cdot H\right) \mid 1 \leq i \leq k\right\}=\operatorname{dim}(H)$.

$(\Rightarrow)$ In order to simplify the proof we may assume that $G$ and $H$ are definable over $\emptyset$, since otherwise we can repeat the argument taking the dimension of the element over a set of parameters $A$, big enough to define both sets. Assume that $H$ has infinite index in $G$. Observe that this implies that $H$ is infinite, because otherwise $\operatorname{dim}(H)=0$. Thus we conclude that $\operatorname{dim}(G)=0$, which implies that $[G: H]$ should be finite.

Consider the equivalence relation $\sim$ over $G$ defined by $g_{1} \sim g_{2}$ if and only if there is an element $h \in H$ such that $g_{1}=g_{2} \cdot h$. Since $H$ is definable, $\sim$ is also definable. By Theorem 7 of [3], the structure $\left(\mathbb{Z}, \mathcal{L}_{\text {Pres }}\right)$ eliminates imaginaries, so there is an $\emptyset$-definable function $\alpha: G \rightarrow G$ such that $\alpha(x)=\alpha(y)$ if and only if $x \sim y$. Suppose that $[G: H]$ is infinite, or equivalently, that $\operatorname{Im}(\alpha)$ is an infinite definable set. By cell decomposition (Theorem 2.2.5) there is a finite partition $\mathcal{P}$ of $\operatorname{Im}(\alpha)$ in definable cells such that $\operatorname{Im}(\alpha)=\bigcup_{i=1}^{n} C_{i}$ and, since $\operatorname{Im}(\alpha)$ is an infinite, at least one of its cells has positive dimension. (Recall that if $\operatorname{dim}(C)=0$ then $C$ is finite) Let $C$ be a cell contained in $\operatorname{Im}(\alpha)$ whose dimension is at least 1 , and pick $a \in C$ such that $\operatorname{dim}(a)>0$.

Claim: there is a generic element $h$ in $H$ over $a$.

Since $H$ is infinite, by compactness we can find a generic element $h \in H$ over $a$. (Follow the idea of Lemma )

Let $h$ be a generic element of $H$ over $a$, then

$$
\operatorname{dim}(h / a)=\operatorname{dim}(H)
$$

Now, define $g=a \cdot h$. We have $a \in d c l(g)$ because $\alpha(g)=a$. On the other hand, 
$h=a^{-1} \cdot g$, which implies that $h \in d c l(a, g)=d c l(g)$. Therefore, by Lemma 2.3.7,

$$
\operatorname{dim}(G) \geq \operatorname{dim}(g) \geq \operatorname{dim}(h, a)=\operatorname{dim}(h / a)+\operatorname{dim}(a)>\operatorname{dim}(H) .
$$

and we are done.

\subsubsection{Open cells and boxes}

Definition 2.3.13. Let $C \subseteq \mathcal{M}^{n}$ be an $A$-definable cell. We say that $C$ is an open cell if $\operatorname{dim}(C)=n$.

Definition 2.3.14 (Box around of a point $\bar{a}$ ). Let $\bar{a}$ be a point in $\mathcal{M}^{n}$, we define a box around $\bar{a}$ inductively:

- A 1-Box around $a$ is a 1-cell $C=\left\{\alpha \square_{1} x \square_{2} \beta \mid x \equiv_{N} c\right\}$ such that $a \in C$ and $[\alpha, a]$ and both $[a, \beta]$ are both infinite.

- Assume that we have defined a $n$-Box $B$ around $\left(a_{1}, \ldots, a_{n}\right)$. Let $C$ be a 1 -box around $a_{n+1}$, then $B \times C$ is an $(n+1)$-box around $\left(a_{1}, \ldots, a_{n+1}\right)$.

Lemma 2.3.15. Let $C$ be an $(1,1)$-cell definable over a set of parameters $B$. Let $(a, b)$ be a dim-generic point of $C$, then there is a definable box $B$, such that $(a, b) \in B \subseteq C$.

Proof. Assume that $C=\left\{(x, t) \mid x \in D \wedge \alpha(x) \leq t \leq \beta(x) \wedge t \equiv_{m} d\right\}$, where $D$ is a 1-cell of the form $D=\left\{\gamma \leq x \leq \delta \mid x \equiv_{n} c\right\}$. Also, we may assume that

$$
\begin{aligned}
& \alpha(x)=s_{1}\left(\frac{x-c_{1}}{k_{1}}\right)+\sigma_{1}, \\
& \beta(x)=s_{2}\left(\frac{x-c_{2}}{k_{2}}\right)+\sigma_{2} .
\end{aligned}
$$

Without loss of generality, we may assume that $\alpha$ and $\beta$ are not constant functions. (If $\alpha$ is constant, then the left end point of the interval around $b$ that we are going to construct 
will be $\alpha)$.

Then we have the following cases:

1. $\beta$ is increasing and $\alpha$ is decreasing,

2. $\beta$ is decreasing and $\alpha$ is increasing,

3. $\beta$ and $\alpha$ are both increasing,

4. $\beta$ and $\alpha$ are both decreasing.

We will show how to solve cases 1 and 3 , since 2 and 4 are respectively similar.

Case 1: Assume that $\beta$ is increasing and $\alpha$ is decreasing.

Then we consider the following type:

$$
\begin{aligned}
\Sigma(x)= & \{x \in D\} \cup\{x<a\} \cup\{\beta(x)>b>\alpha(x)\} \cup\{x \neq d \mid d \in D \cap \operatorname{dcl}(B a) \wedge d<a\} \\
& \cup\left\{\exists y_{1} \ldots y_{n}\left(\bigwedge_{i=1}^{n}\left(b<y_{i}<\beta(x)\right) \wedge \bigwedge_{i \neq j}\left(y_{i} \neq y_{j}\right)\right): n \in \omega\right\} \\
& \cup\left\{\exists y_{1} \ldots y_{n}\left(\bigwedge_{i=1}^{n}\left(\alpha(x)<y_{i}<b\right) \wedge \bigwedge_{i \neq j}\left(y_{i} \neq y_{j}\right)\right): n \in \omega\right\}
\end{aligned}
$$

Claim: $\Sigma(x)$ is consistent

By compactness, it is enough to show that it is finitely consistent. Let $\Sigma_{0}(x) \subset_{f i n} \Sigma(x)$, and fix $d_{1}, \ldots, d \in D \cap d c l(B a)$.Assume that:

$$
\begin{aligned}
\Sigma_{0}(x) & =\{x \in D\} \cup\{x<a\} \cup\{\beta(x)>b>\alpha(x)\} \cup\left\{x \neq d_{i}: 1 \leq i \leq l\right\} \\
& \cup\left\{\exists y_{1} \ldots y_{n}\left(\bigwedge_{i=1}^{n}\left(b<y_{i}<\beta(x)\right) \wedge \bigwedge_{i \neq j}\left(y_{i} \neq y_{j}\right)\right)\right\} \\
& \cup\left\{\exists y_{1} \ldots y_{m}\left(\bigwedge_{i=1}^{m}\left(\alpha(x)<y_{i}<b\right) \wedge \bigwedge_{i \neq j}\left(y_{i} \neq y_{j}\right)\right)\right\}
\end{aligned}
$$

Now consider the following cases: 
- There is some $i$ such that $\beta\left(d_{i}\right)>b>\alpha\left(d_{i}\right)$.

In this case, take $d=\min \left\{d_{i} \mid \beta\left(d_{i}\right)>b>\alpha\left(d_{i}\right)\right\}$. Then, take $d-n<d<a$ and notice that $d-n \in D$, because $d-n \equiv_{n} c$ since $d \equiv_{n} c$, and $\gamma \leq d-n<d<a<\delta$ (If $d=\gamma$ then we can take the box $[\gamma, \delta] \times[\alpha(\gamma), \beta(\gamma)])$. Also, since $\beta$ is increasing then $\beta(d-n)<\beta(d)$, but this implies that $b<\beta(d-n)$, because otherwise $\beta(d-n)<$ $b<\beta(d)$, which implies that $b \in \operatorname{dcl}(B a)$ since $[\beta(d-n), \beta(d)]$ is finite. Additionally, observe that $[b, \beta(d-n)]$ should be infinite, because if it is finite then $b \in \operatorname{dcl}(B a)$, contradicting the fact that $(a, b)$ is a generic point of $C$. Using that $\alpha$ is decreasing, similarly we can conclude that $\alpha(d-n)<b$ and, moreover, $[\alpha(d-n), b]$ is infinite. Let $m=\max \left\{d_{i} \mid \beta\left(d_{i}\right)<b\right\} \cup\left\{d_{i} \mid \alpha\left(d_{i}\right)>b\right\}$, then if $\beta(m)<b<\beta(d-n)$, since $\beta$ is increasing, we deduce that $m<d-n$, which implies that $d-n \neq d_{i}$ for all $1 \leq i \leq l$. If $\alpha(m)>b$, the argument follows similarly using the fact that $\alpha$ is decreasing. Then, $\mathcal{M} \vDash \Sigma_{0}(d-n)$.

- For each $1 \leq i \leq l, \beta\left(d_{i}\right)<b$ or $\alpha\left(d_{i}\right)>b$.

Note that $a-n \in D$ and $a-n<a$ and $\beta(a-n)>b>\alpha(a-n)$. In fact $\alpha(a)<\alpha(a-n)<\beta(a-n)<\beta(a)$, and $b \notin[\alpha(a), \alpha(a-n)]$ and $b \notin[\beta(a-n), \beta(a)]$ (since each of these intervals is finite). Therefore, since $b \in[\alpha(a), \beta(a)]$ we have $\beta(a-n)>b>\alpha(a-n)$. Moreover, the algebraic independence of $b$ over $B a$ implies also that $[\alpha(a-n), b]$ and $[b, \alpha(a)]$ are both infinite. Thus $\mathcal{M} \vDash \Sigma_{0}(a-n)$.

We conclude that $\Sigma(x)$ is consistent, then by saturation of $\mathcal{M}$, there is some $c \in \mathcal{M} \vDash \Sigma(c)$. Then let:

$$
\begin{aligned}
& I_{1}=\left\{c \leq x \leq \delta \mid x \equiv_{n} c\right\} \\
& I_{2}=\left\{\alpha(c) \leq t \leq \beta(c) \mid t \equiv_{m} d\right\}
\end{aligned}
$$

By construction $(a, b) \in I_{1} \times I_{2}$ and it is a box around $(a, b)$. So, it is enough to show that $I_{1} \times I_{2} \subseteq C$. If $x \in I_{1}$, then $c \leq x$, which implies $\beta(c) \leq \beta(x)$ and $\alpha(x) \leq \alpha(c)$. Then, 
$I_{1} \times I_{2} \subseteq C$

Case 2: Assume now that $\beta$ and $\alpha$ are both increasing.

Then we can consider the following types:

$$
\begin{aligned}
\Sigma_{1}(x) & =\{x \in C\} \cup\{x<a\} \cup\{\beta(x)>b\} \cup\{(x \neq d): d \in d \operatorname{cl}(B a) \cap C \wedge d<a\} \\
& \left.\cup\left\{\exists y_{1}, \ldots, y_{n}\left(\bigwedge_{i=1}^{n} b<y_{i}<\beta(x)\right) \wedge\left(\bigwedge_{i \neq j} y_{i} \neq y_{j}\right)\right): n<\omega\right\} \\
\Sigma_{2}(x)= & \{x \in C\} \cup\{x>a\} \cup\{\alpha(x)<b\} \cup\{(x \neq d): d \in d \operatorname{dcl}(B a) \cap C \wedge d>a\} \\
& \left.\cup\left\{\exists y_{1}, \ldots, y_{n}\left(\bigwedge_{i=1}^{n} \alpha(x)<y_{i}<b\right) \wedge\left(\bigwedge_{i \neq j} y_{i} \neq y_{j}\right)\right): n \in \omega\right\}
\end{aligned}
$$

Similarly as in the previous case we can show that $\Sigma_{1}(x)$ and $\Sigma_{2}(x)$ are both consistent. By saturation, we can take $c, d \in \mathcal{M}$ such that $\mathcal{M} \vDash \Sigma_{1}(c)$ and $\mathcal{M} \vDash \Sigma_{2}(d)$. Then, define the following 1-cells:

$$
\begin{aligned}
& I_{1}=\left\{c \leq x \leq d \mid x \equiv_{n} c\right\} \\
& I_{2}=\left\{\alpha(d) \leq t \leq \beta(c) \mid t \equiv_{m} d\right\}
\end{aligned}
$$

Then $(a, b) \in I_{1} \times I_{2}$, and by construction $I_{1} \times I_{2}$ is a box around $(a, b)$. Also, it is clear that $I_{1} \subseteq D$, and notice that for every element $x \in I_{1}, c \leq x \leq d$, then $\beta(c) \leq \beta(x)$ and $\alpha(x) \leq \alpha(d)$, then $I_{1} \times I_{2} \subseteq C$.

Lemma 2.3.16. Let $C$ be an open $n$-cell definable over $B$ and $\bar{a}$ a dim-generic element of $C$. Then, we can find an $n$-box $B$ such that $\bar{a} \in B \subseteq C$.

Proof. We proceed by induction on $n$.

Suppose first that $n=1$. Let $C=\left\{\alpha \square_{1} x \square_{2} \beta \mid x \equiv_{N} c\right\}$, where $\alpha, \beta \in \operatorname{dcl}(B)$. Since $\operatorname{dim}(a / B)=1$, then $a \notin \operatorname{dcl}(B)$, then $[\alpha, a]$ and $[a, \beta]$ are both infinite. Therefore, $C$ is a box around $a$.

Induction Hypothesis: Let $Y$ be an open $n$-cell and $\left(a_{1}, \ldots, a_{n}\right) \in Y$ a dim-generic 
point. Then, there is an $n$-box $B$ around $\left(a_{1}, \ldots, a_{n}\right)$ such that $B \subseteq Y$

Let $C$ be an open $(n+1)$-cell, say of the form:

$$
\left\{(\bar{x}, t) \mid \bar{x} \in D \wedge \alpha(\bar{x}) \leq t \leq \beta(\bar{x}) \wedge t \equiv_{m} c\right\}
$$

where $D$ is an $n$-open cell and

$$
\begin{aligned}
& \alpha(\bar{x})=\sum_{i=1}^{n} s_{i}\left(\frac{x_{i}-c_{i}}{N_{i}}\right)+\gamma_{1} \\
& \beta(\bar{x})=\sum_{i=1}^{n} r_{i}\left(\frac{x_{i}-d_{i}}{M_{i}}\right)+\gamma_{2}
\end{aligned}
$$

and take an element $\left(a_{1}, \ldots, a_{n+1}\right)$ a dim-generic point of $C$. Then $D=\pi(C)$ is an open $n$-cell and $\left(a_{1}, \ldots, a_{n}\right)$ should be a dim-generic point of $Y$. Then, by the induction hypothesis there a box $S$ around $\left(a_{1}, \ldots, a_{n}\right)$ such that $S \subseteq D$.

Assume that $S=I_{1} \times \cdots \times I_{n}$, and that for each $1 \leq i \leq n, I_{i}=\left\{\delta_{1}^{i} \leq x \leq \delta_{2}^{i} \mid x \equiv_{k_{i}} l_{i}\right\}$. Now define the following lines inside $S$ :

$$
\begin{aligned}
& L_{1}=I_{1} \times\left\{a_{2}\right\} \times \cdots \times\left\{a_{n}\right\} \\
& \vdots \\
& L_{n}=\left\{a_{1}\right\} \times \cdots \times\left\{a_{n-1}\right\} \times I_{n}
\end{aligned}
$$

Observe that $a_{i} \in L_{i}$ for every $1 \leq i \leq n$, and the restriction of $\alpha(\bar{x})$ and $\beta(\bar{x})$ to $L_{i}$ are lines. We will indicate as $\alpha_{i}$ and $\beta_{i}$ those restrictions. For example, the restriction of $\alpha(\bar{x})$ 
and $\beta(\bar{x})$ to $L_{1}$ are:

$$
\begin{aligned}
& \alpha\left(x_{1}\right)=s_{1}\left(\frac{x_{1}-c_{1}}{N_{1}}\right)+\underbrace{\sum_{i=2}^{n}\left(\frac{a_{i}-c_{i}}{N_{i}}\right)+\gamma_{1}}_{\in d c l\left(A, a_{2}, \ldots, a_{n}\right)}, \\
& \beta\left(x_{1}\right)=s_{1}\left(\frac{x_{1}-d_{1}}{M_{1}}\right)+\underbrace{\sum_{i=2}^{n}\left(\frac{a_{i}-d_{i}}{M_{i}}\right)+\gamma_{1}}_{\in d c l\left(A, a_{2}, \ldots, a_{n}\right)} .
\end{aligned}
$$

In order to simplify the notation, we will assume that all of these restrictions of $\beta$ are increasing and all of the restriction of $\alpha$ are increasing.

Additionally, since $\operatorname{dim}\left(a_{n+1} / B a_{1}, \ldots, a_{n}\right)=1$, following the argument of Lemma 2.3.15 applied to each $L_{i}$ and $\alpha_{i}(x)=\alpha(x) \uparrow_{L_{i}}, \beta_{i}(x)=\beta(x) \uparrow_{L_{i}}$, we can find $\delta_{1}^{i} \leq c_{i}<a_{i}<d_{i} \leq$ $\delta_{2}^{i}$ such that:

- The intervals $\left[c_{i}, a_{i}\right]$ and $\left[a_{i}, d_{i}\right]$ are both infinite.

- The intervals $\left[a_{n+1}, \beta_{i}\left(c_{i}\right)\right]$ and $\left[\alpha_{i}\left(d_{i}\right), a_{n+1}\right]$ are infinite,

- The set $\left\{c_{i} \leq x \leq d_{i} \mid x \equiv_{k_{i}} l_{i}\right\} \times\left\{\alpha_{i}\left(d_{i}\right) \leq t \leq \beta_{i}\left(c_{i}\right) \mid t \equiv_{m} c\right\}$ is a 2-box and it is a subset of the 2-open cell

$$
\left\{(x, t) \mid x \in I_{1} \wedge \alpha \uparrow_{L_{i}}(x) \leq t \leq \beta \uparrow_{L_{i}}(x) \wedge t \equiv_{m} c\right\}
$$

Define $J_{i}=\left\{c_{i} \leq x \leq d_{i} \mid x \equiv_{k_{i}} l_{i}\right\}$ and notice that $a_{i} \in J_{i} \subseteq I_{i}$. Also, take $m=\max \left\{\alpha_{i}\left(d_{i}\right) \mid 1 \leq i \leq n\right\}$ and $M=\min \left\{\beta_{i}\left(c_{i}\right) \mid 1 \leq i \leq n\right\}$. Observe that,

$$
B=J_{1} \times \cdots \times J_{n} \times\left\{t \in \mathcal{M}|m \leq t \leq M| t \equiv_{m} c\right\}
$$

Then, $B$ is a box around $\left(a_{1}, \ldots, a_{n}\right)$ and $B \subseteq C$, as we desired.

Lemma 2.3.17. Let $B, C$ be two boxes around a point $\bar{a}$, then $B \cap C$ is a box around $\bar{a}$. 
Proof. Assume that $B=I_{1} \times \cdots \times I_{n}$ and $C=J_{1} \times \cdots \times J_{n}$ are $n$-boxes around a point $\bar{a}=\left(a_{1}, \ldots, a_{n}\right) \in \mathcal{M}^{n}$. Assume that:

$$
\begin{gathered}
I_{1}=\left\{b_{1}^{1} \leq x \leq b_{2}^{1} \mid x \equiv_{N_{1}} d_{1}\right\}, \\
\vdots \\
I_{n}=\left\{b_{1}^{n} \leq x \leq b_{2}^{n} \mid x \equiv_{N_{n}} d_{n}\right\} .
\end{gathered}
$$

And,

$$
\begin{gathered}
J_{1}=\left\{c_{1}^{1} \leq x \leq c_{2}^{1} \mid x \equiv_{M_{1}} e_{1}\right\}, \\
\vdots \\
J_{n}=\left\{c_{1}^{n} \leq x \leq c_{2}^{n} \mid x \equiv_{M_{n}} e_{n}\right\} .
\end{gathered}
$$

Since for each $i \leq n, a_{i} \in I_{i} \cap J_{i}$, then each linear system $x \equiv_{N_{i}} d_{i}$ and $x \equiv_{M_{i}} e_{i}$ is consistent, then it can be reduced to a unique equation $x \equiv_{L_{i}} f_{i}$. For each $i \leq n$, take $\alpha_{i}=\max \left\{c_{1}^{i}, b_{1}^{i}\right\}$ and $\beta_{i}=\min \left\{c_{2}^{i}, b_{2}^{i}\right\}$ and define:

$$
\begin{aligned}
& S_{1}=\left\{\alpha_{1} \leq x \leq \beta_{1} \mid x \equiv_{L_{1}} f_{1}\right\}, \\
& \quad \vdots \\
& S_{n}=\left\{\alpha_{n} \leq x \leq \beta_{n} \mid x \equiv_{L_{n}} f_{n}\right\} .
\end{aligned}
$$

Then $B \cap C=S_{1} \times \cdots \times S_{n}$, and since $B$ and $C$ are boxes around $\bar{a}$ we have that for every $i \leq n,\left[\alpha_{i}, a_{i}\right]$ and $\left[a_{i}, \beta_{i}\right]$ are both infinite. Therefore, $B \cap C$ is a $n$-box around the point $\bar{a}$. 


\section{Chapter 3}

\section{A characterization of definable}

\section{groups in Presburger Arithmetic}

Let $\left(G, \cdot, e_{G}\right)$ be a definable group in Presburger Arithmetic and assume that $G(\mathcal{M}) \subseteq$ $\mathcal{M}^{n}$. Consider $\left(\mathcal{M}^{n},+, 0\right)$ as a group with the vectorial addition, which is defined as:

$$
\left(x_{1}, \ldots, x_{n}\right)+\left(y_{1}, \ldots, y_{n}\right)=\left(x_{1}+y_{1}, \ldots, x_{n}+y_{n}\right)
$$

The aim of this section is to establish a relationship between the operation $\cdot$ and + .

\subsection{Group operation vs usual addition}

The main purpose of this section is to show that for every definable group $G$ there is a an open box $B_{a} \subseteq G$, such that for every $\bar{x}, \bar{y} \in B_{\bar{a}}$, we have that:

$$
\bar{x} \cdot \bar{a}^{-1} \cdot \bar{y}=\bar{x}-\bar{a}+\bar{y} .
$$

Our proof is based on the work of Eleftheriou and Starchenko in [13]. In fact they proved this result for definable groups definable in vector spaces over division rings, and their 
statements use mainly the cell decomposition for those structures. We develop a similar theorem based on their work, using the cell decomposition for Presburger Arithmetic.

In the following statements we will assume that $\operatorname{dim}(G)=n$. Additionally, we suppose that $G$ is definable over a small model $\mathcal{M}_{0}$.

Lemma 3.1.1. Let $\bar{a}, \bar{b} \in G$ be two dim-generic and independent points. Then, there are $n$-boxes $B_{\bar{a}}$ and $B_{\bar{b}}$ around $\bar{a}$ and $\bar{b}$ respectively, and $\mathcal{M}_{0}$-definable linear functions $f_{1}, \ldots, f_{n}$ defined on $B_{\bar{a}} \times B_{\bar{b}}$ such that for every $(\bar{x}, \bar{y}) \in B_{\bar{a}} \times B_{\bar{b}}$, we have that

$$
\bar{x} \cdot \bar{y}=\left(f_{1}(\bar{x}, \bar{y}), \ldots, f_{n}(\bar{x}, \bar{y})\right)
$$

Proof. Since the multiplication of the group $G$ is a definable function, then for every $i \leq n$ the map obtained by composing with the projection on the $i$-th coordinate, $\pi_{i} \circ \cdot$, is $A$ definable. By cell decomposition, there is a finite partition $\mathcal{P}_{i}$ of $G \times G$ in $\mathcal{M}_{0^{-}}$-cells, such that each function $\pi_{i} \circ$. is linear. Moreover, by Lemma 2.2.6, we can find a partition $\mathcal{P}$ in cells of $G$ such that for every $i \leq n$ and $C \in \mathcal{P}, \pi_{i} \circ \cdot \Gamma_{C}$ is an $\mathcal{M}_{0}$-definable linear function.

Thus, there is some cell $C \in \mathcal{P}$, such that $(\bar{a}, \bar{b}) \in C$. Since $\bar{a}, \bar{b}$ are independent, we have $\operatorname{dim}(\bar{a}, \bar{b})=n+n \leq \operatorname{dim}(C) \leq \operatorname{dim}(G \times G)=2 n$, so $\operatorname{dim}(C)=2 n$. Since $\operatorname{dim}((\bar{a}, \bar{b}) / A)=2 n$, by Lemma 2.3.16, there is a $2 n$-box $B$ around the point $(\bar{a}, \bar{b})$ completely contained in $C$. Assume that $B=I_{1} \times \cdots \times I_{n} \times I_{n+1} \times \cdots \times I_{2 n}$, then $B_{\bar{a}}=I_{1} \times \cdots \times I_{n}$ and $B_{\bar{b}}=I_{n+1} \times \cdots \times I_{2 n}$ are boxes around $\bar{a}$ and $\bar{b}$ respectively. Therefore, $B_{\bar{a}} \times B_{\bar{b}}=B \subseteq C$, and for every $(\bar{x}, \bar{y}) \in B_{\bar{a}} \times B_{\bar{b}}$, there are $\mathcal{M}_{0^{0}}$-linear functions $f_{1}, \ldots, f_{n}$ such that:

$$
\bar{x} \cdot \bar{y}=\left(f_{1}(\bar{x}, \bar{y}), \ldots, f_{n}(\bar{x}, \bar{y})\right) .
$$

Lemma 3.1.2. For every two dim-generic and independent points $\bar{a}, \bar{b} \in G$, there are 
$n$-boxes $B_{\bar{a}}$ and $B_{\bar{b}}$ around $\bar{a}$ and $\bar{b}$ respectively, completely contained in $G$ such that:

- For all $x \in B_{\bar{a}}$, we have that $\bar{x} \cdot \bar{a}^{-1} \cdot \bar{b}=\left(h_{1}(\bar{x}), \ldots, h_{n}(\bar{x})\right) \in B_{\bar{b}}$, where each $h_{i}$ is a $\mathcal{M}_{0} \cup\left\{\bar{a}^{-1} \cdot \bar{b}\right\}$-linear function,

- For every $x \in B_{\bar{a}}$, we have that $\bar{a}^{-1} \cdot \bar{b} \cdot \bar{x}=\left(g_{1}(\bar{x}), \ldots, g_{n}(\bar{x})\right) \in B_{\bar{b}}$, where each $g_{i}$ is a $\mathcal{M}_{0} \cup\left\{\bar{a}^{-1} \cdot \bar{b}\right\}$-linear function.

Proof. Since $\bar{a}$ and $\bar{b}$ are independent and dim-generics of $G$, then $\bar{a}$ and $\bar{a}^{-1} \cdot \bar{b}$ are independent and dim-generics of $G$ as well. Therefore, by Lemma 3.1.1 there are boxes $B_{\bar{a}}^{\prime}$ and $B_{\bar{a}^{-1 . \bar{b}}}$, such that for all $\bar{x} \in B_{\bar{a}}^{\prime}$ and $\bar{y} \in B_{\bar{a}^{-1} \cdot \bar{b}}$ we have that

$$
\bar{x} \cdot \bar{y}=\left(f_{1}(\bar{x}, \bar{y}), \ldots, f_{n}(\bar{x}, \bar{y})\right),
$$

where each $f_{i}$ is an $\mathcal{M}_{0}$-linear function. Then, for each $1 \leq i \leq n$ we have

$$
f_{i}(\bar{x}, \bar{y})=\sum_{j=1}^{n} s_{j}^{i}\left(\frac{x_{j}-c_{j}^{i}}{k_{j}^{i}}\right)+\sum_{j=1}^{n} r_{j}^{i}\left(\frac{y_{j}-d_{j}^{i}}{m_{j}^{i}}\right)+\gamma_{i},
$$

where, for each $1 \leq j \leq n, 0 \leq c_{j}^{i}<k_{j}^{i}$ and $s_{j}$ are integers and $\gamma_{i} \in \mathcal{M}_{0}$. Therefore, for each $1 \leq i \leq n$, and $\bar{x} \in B_{\bar{a}}^{\prime}$ :

$$
\left(\bar{x} \cdot \bar{a}^{-1} \cdot \bar{b}\right)_{i}=\sum_{j=1}^{n} s_{j}^{i}\left(\frac{x_{j}-c_{j}^{i}}{k_{j}^{i}}\right)+\sum_{j=1}^{n} r_{j}^{i}\left(\frac{\left(\bar{a}^{-1} \cdot \bar{b}\right)_{j}-d_{j}^{i}}{m_{j}^{i}}\right)+\gamma_{i}
$$

Then, putting $\beta_{i}=\sum_{j=1}^{n} r_{j}^{i}\left(\frac{\left(\bar{a}^{-1} \cdot \bar{b}\right)_{j}-d_{j}^{i}}{m_{j}^{i}}\right)+\gamma_{i}$, we have that for all $\bar{x} \in B_{\bar{a}}^{\prime}$,

$$
\left(\bar{x} \cdot \bar{a}^{-1} \cdot \bar{b}\right)_{i}=\sum_{j=1}^{n} s_{j}^{i}\left(\frac{x_{j}-c_{j}^{i}}{k_{j}^{i}}\right)+\beta_{i}
$$

which is an $\mathcal{M}_{0} \cup\left\{\bar{a}^{-1} \cdot \bar{b}\right\}$-linear function. 
Consider now $A_{\bar{b}}=\left\{\bar{x} \cdot \bar{a}^{-1} \cdot \bar{b} \mid \bar{x} \in B_{\bar{a}}^{\prime}\right\}$, which is an $\mathcal{M}_{0} \cup\left\{\bar{a}^{-1} \cdot \bar{b}\right\}$-definable set. Also, notice that $\bar{b}=\bar{a} \cdot \bar{a}^{-1} \cdot \bar{b}$, therefore $\bar{b} \in A_{\bar{b}}$. By cell decomposition $A_{\bar{b}}$ is a finite union of $\mathcal{M}_{0} \cup\left\{\bar{a}^{-1} \cdot \bar{b}\right\}$-cells, so there is some cell $D$ such that $\bar{b} \in D$, and $\operatorname{dim}(D)$ should be equal to $n$, since $\bar{b}$ and $\bar{a}^{-1} \cdot \bar{b}$ are also independent. By Lemma 2.3.16, there is a box $B_{\bar{b}}^{\prime}$ around $\bar{b}$ such that $B_{\bar{b}}^{\prime} \subseteq D \subseteq A_{\bar{b}}$. Now, define $A_{\bar{a}}=\left\{\bar{x} \in B_{\bar{a}}^{\prime} \mid \bar{x} \cdot \bar{a}^{-1} \cdot \bar{b} \in B_{\bar{b}}^{\prime}\right\}$, which is an $\mathcal{M}_{0} \cup\left\{\bar{a}^{-1} \cdot \bar{b}\right\}$-definable set and $\bar{a} \in A_{\bar{a}}$. Again we can find an $n$ - box $B_{\bar{a}}^{\prime}$ around $\bar{a}$ such that $B_{\bar{a}}^{\prime} \subseteq A_{\bar{a}}$.

Observe that these boxes satisfy the first condition of the lemma.

Similarly, we can obtain two $n$-boxes $B_{\bar{a}}^{\prime \prime}, B_{\bar{b}}^{\prime \prime}$ which satisfy the second equality. Now, take $B_{\bar{a}}=B_{\bar{a}}^{\prime} \cap B_{\bar{a}}^{\prime \prime}$ and $B_{\bar{b}}=B_{\bar{b}}^{\prime} \cap B_{\bar{b}}^{\prime \prime}$, which are boxes since the intersection of two boxes around a point is also a box, and by construction they satisfy the desired conditions.

Lemma 3.1.3. Let $\bar{a}$ be a dim-generic element, then there is an $n$-box $B_{\bar{a}}$ around $\bar{a}$, such that for all $\bar{x}, \bar{y} \in B_{\bar{a}}$ and for each $i \leq n$ :

$$
\left(\bar{x} \cdot \bar{a}^{-1} \cdot \bar{y}\right)_{i}=h_{i}\left(\left(f_{1}(\bar{x}), \ldots, f_{n}(\bar{x})\right),\left(g_{1}(\bar{y}), \ldots, g_{n}(\bar{y})\right)\right)
$$

where:

- Each $h_{i}$ is an $\mathcal{M}_{0}$-linear function.

- Each $f_{j}$ and $g_{j}$ are $\mathcal{M}_{0} \cup\left\{\bar{a}, \bar{a}_{1}\right\}$-definable functions, with $\bar{a}_{1}$ a dim-generic element of $G$ independent from $\bar{a}$.

Proof. Let $\bar{a}$ be a dim-generic element of $G$ and take $\bar{a}_{1}$ a dim-generic element of $G$ independent of $\bar{a}$. Consider $\bar{a}_{2}=\bar{a} \cdot \bar{a}_{1}^{-1}$, which is also dim-generic and independent of $\bar{a}$. Applying Lemma 3.1.2 to $\bar{a}$ and $\bar{a}_{1}$ we obtain two $n$-boxes $B_{\bar{a}}^{\prime}$ and $B_{\bar{a}_{1}}^{\prime}$ such that for all $\bar{x} \in B_{\bar{a}}^{\prime}$ and each $1 \leq i \leq n$ we have:

$$
\left(\bar{x} \cdot \bar{a}^{-1} \cdot \bar{a}_{1}\right)_{i=1}^{n}=\left(f_{i}(\bar{x})\right)_{i=1}^{n} \in B_{\bar{a}_{1}}^{\prime}
$$


where each $f_{i}$ is $\mathcal{M}_{0} \cup\left\{\bar{a}^{-1} \cdot \bar{a}_{1}\right\}$-definable and linear.

Similarly, since $\bar{a}$ and $\bar{a}_{2}$ are also independent and dim-generics, there are two $n$-boxes $B_{\bar{a}}^{\prime \prime}$ and $B_{\bar{a}_{2}}^{\prime \prime}$ such that for all $\bar{x} \in B_{\bar{a}}^{\prime \prime}$ and each $1 \leq i \leq n$ :

$$
\left(\bar{a}^{-1} \cdot \bar{a}_{2} \cdot \bar{x}\right)_{i=1}^{n}=\left(g_{i}(\bar{x})\right)_{i=1}^{n} \in B_{\bar{a}_{2}}^{\prime \prime},
$$

where each $g_{i}$ is $\mathcal{M}_{0} \cup\left\{\bar{a}^{-1} \cdot \bar{a}_{2}\right\}$-definable and linear. Additionally, applying Lemma 3.1.1 to $\bar{a}_{1}$ and $\bar{a}_{2}$, which are also independent and dim-generics, there are $n$-boxes $C_{\bar{a}_{1}}$ and $C_{\bar{a}_{2}}$ such that for all $\bar{x} \in C_{\bar{a}_{1}}$ and $\bar{y} \in C_{\bar{a}_{2}}$ we have that:

$$
\bar{x} \cdot \bar{y}=\left(h_{i}(\bar{x}, \bar{y})\right)_{i=1}^{n},
$$

where each function $h_{i}$ is an $\mathcal{M}_{0}$-definable linear function.

Now we can consider the $n$-boxes $B_{\bar{a}}=B_{\bar{a}}^{\prime} \cap B_{\bar{a}}^{\prime \prime}, B_{\bar{a}_{1}}=C_{\bar{a}_{1}} \cap B_{\bar{a}_{1}}^{\prime}$ and $B_{\bar{a}_{2}}=C_{\bar{a}_{2}} \cap B_{\bar{a}_{2}}^{\prime \prime}$. Summarizing, for every $\bar{x}, \bar{y} \in B_{\bar{a}}$, we have that $\left(\bar{x} \cdot \bar{a}^{-1} \cdot \bar{a}_{1}\right)_{i=1}^{n}=\left(f_{i}(\bar{x})\right)_{i=1}^{n} \in B_{\bar{a}_{1}}$, $\left(\bar{a}^{-1} \cdot \bar{a}_{2} \cdot \bar{y}\right)_{i=1}^{n}=\left(g_{i}(\bar{y})\right)_{i=1}^{n} \in B_{\bar{a}_{2}}$ and for all $\bar{z} \in B_{\bar{a}_{1}}, \bar{w} \in B_{\bar{a}_{2}}$ it holds that

$$
\bar{z} \cdot \bar{w}=\left(h_{j}(\bar{z}, \bar{w})\right)_{j=1}^{n} .
$$

Now, let $\bar{x}, \bar{y} \in B_{\bar{a}}=B_{\bar{a}}^{\prime} \cap B_{\bar{a}}^{\prime \prime}$. Notice that:

$$
\begin{aligned}
\bar{x} \cdot \bar{a}^{-1} \cdot \bar{y} & =\bar{x} \cdot \bar{a}^{-1} \cdot \bar{a}_{1} \cdot \bar{a}_{1}^{-1} \cdot \bar{y} \\
& =\left(\bar{x} \cdot \bar{a}^{-1} \cdot \bar{a}_{1}\right) \cdot\left(\bar{a}_{1}^{-1} \cdot \bar{y}\right) \\
& =\underbrace{\left(\bar{x} \cdot \bar{a}^{-1} \cdot \bar{a}_{1}\right)}_{\in B_{\bar{a}_{1}}} \cdot \underbrace{\left(\bar{a}^{-1} \cdot \bar{a}_{2} \cdot \bar{y}\right)}_{\in B_{\bar{a}_{2}}}
\end{aligned}
$$


Thus, for each $j \leq n$ :

$$
\begin{aligned}
\left(\bar{x} \cdot \bar{a}^{-1} \cdot \bar{y}\right)_{j} & =\left(\left(\bar{x} \cdot \bar{a}^{-1} \cdot \bar{a}_{1}\right) \cdot\left(\bar{a}^{-1} \cdot \bar{a}_{2} \cdot \bar{y}\right)\right)_{j}, \\
& =h_{j}\left(\left(\bar{x} \cdot \bar{a}^{-1} \cdot \bar{a}_{1}\right),\left(\bar{a}^{-1} \cdot \bar{a}_{2} \cdot \bar{y}\right)\right), \\
& =h_{j}\left(\left(f_{1}(\bar{x}), \ldots, f_{n}(\bar{x})\right),\left(g_{1}(\bar{y}), \ldots, g_{n}(\bar{y})\right)\right),
\end{aligned}
$$

as required.

Lemma 3.1.4. Let $\bar{a}$ be a dim-generic element of $G$. Then there is an $n$-box $B_{\bar{a}}$ around $\bar{a}$, such that for every $\bar{x}, \bar{y} \in B_{\bar{a}}$, we have that

$$
\bar{x} \cdot \bar{a}^{-1} \cdot \bar{y}=\bar{x}-\bar{a}+\bar{y} .
$$

By Lemma 3.1.3 there is an $n$-box $B_{\bar{a}}$ around $\bar{a}$, such that for every $\bar{x}, \bar{y} \in B_{\bar{a}}$ :

$$
\left(\bar{x} \cdot \bar{a}^{-1} \cdot \bar{y}\right)_{j=1}^{n}=\left(h_{j}\left(\left(f_{1}(\bar{x}), \ldots, f_{n}(\bar{x})\right),\left(g_{1}(\bar{y}), \ldots, g_{n}(\bar{y})\right)\right)\right)_{j=1}^{n}
$$

In particular $\bar{a} \in B_{\bar{a}}$, so we have:

$$
\bar{x}=\bar{x} \cdot \bar{a} \cdot \bar{a}^{-1}=\left(h_{j}\left(\left(f_{1}(\bar{x}), \ldots, f_{n}(\bar{x})\right),\left(g_{1}(\bar{a}), \ldots, g_{n}(\bar{a})\right)\right)\right)_{j=1}^{n} .
$$

Similarly,

$$
\bar{y}=\bar{a} \cdot \bar{a}^{-1} \cdot \bar{y}=\left(h_{j}\left(\left(f_{1}(\bar{a}), \ldots, f_{n}(\bar{a})\right),\left(g_{1}(\bar{y}), \ldots, g_{n}(\bar{y})\right)\right)\right)_{j=1}^{n} .
$$

Fix some $j \leq n$, then:

$$
\begin{aligned}
(\bar{x}+\bar{y})_{j} & =h_{j}\left(\left(f_{1}(\bar{x}), \ldots, f_{n}(\bar{x})\right),\left(g_{1}(\bar{a}), \ldots, g_{n}(\bar{a})\right)\right) \\
& +h_{j}\left(\left(f_{1}(\bar{a}), \ldots, f_{n}(\bar{a})\right),\left(g_{1}(\bar{y}), \ldots, g_{n}(\bar{y})\right)\right) .
\end{aligned}
$$


By construction, we know that

$$
h_{j}(\bar{w}, \bar{z})=\sum_{i=1}^{n} s_{i}^{j}\left(\frac{w_{i}-c_{i}^{j}}{k_{i}^{j}}\right)+\sum_{i=1}^{n} r_{i}^{j}\left(\frac{z_{i}-d_{i}^{j}}{m_{i}^{j}}\right)+\gamma_{j} .
$$

Replacing this equations in (3.1) and (3.2), we conclude that:

$$
\begin{aligned}
(\bar{x}+\bar{y})_{j} & =\sum_{i=1}^{n} s_{i}^{j}\left(\frac{f_{i}(\bar{x})-c_{i}^{j}}{k_{i}^{j}}\right)+\sum_{i=1}^{n} r_{i}^{j}\left(\frac{g_{i}(\bar{a})-d_{i}^{j}}{m_{i}^{j}}\right)+\gamma_{j} \\
& +\sum_{i=1}^{n} s_{i}^{j}\left(\frac{f_{i}(\bar{a})-c_{i}^{j}}{k_{i}^{j}}\right)+\sum_{i=1}^{n} r_{i}^{j}\left(\frac{g_{i}(\bar{y})-d_{i}^{j}}{m_{i}^{j}}\right)+\gamma_{j}
\end{aligned}
$$

Therefore,

$$
\begin{aligned}
(\bar{x}+\bar{y})_{j} & =\underbrace{\sum_{i=1}^{n} s_{i}^{j}\left(\frac{f_{i}(\bar{x})-c_{i}^{j}}{k_{i}^{j}}\right)+\sum_{i=1}^{n} r_{i}^{j}\left(\frac{g_{i}(\bar{y})-d_{i}^{j}}{m_{i}^{j}}\right)+\gamma_{j}}_{\left(x \cdot a^{-1} \cdot y\right)_{j}} \\
& +\underbrace{\sum_{i=1}^{n} s_{i}^{j}\left(\frac{f_{i}(\bar{a})-c_{i}^{j}}{k_{i}^{j}}\right)+\sum_{i=1}^{n} r_{i}^{j}\left(\frac{g_{i}(\bar{a})-d_{i}^{j}}{m_{i}^{j}}\right)+\gamma_{j}}_{\left(\bar{a}^{-} \cdot \bar{a}^{-1} \cdot \bar{a}\right)_{j}}
\end{aligned}
$$

And notice that $\bar{a} \cdot \bar{a}^{-1} \cdot \bar{a}=\bar{a}$, then:

$$
\begin{aligned}
(\bar{x}+\bar{y})_{j} & =\left(\bar{x} \cdot \bar{a}^{-1} \cdot \bar{y}\right)_{j}+\left(\bar{a} \cdot \bar{a}^{-1} \cdot \bar{a}\right)_{j} \\
& =\left(\bar{x} \cdot \bar{a}^{-1} \cdot \bar{y}\right)_{j}+(\bar{a})_{j}
\end{aligned}
$$

Then, $(\bar{x}-\bar{a}+\bar{y})_{j}=\left(\bar{x} \cdot \bar{a}^{-1} \cdot \bar{y}\right)_{j}$, and since this is true for each $j \leq n$, we conclude that

$$
\bar{x}-\bar{a}+\bar{y}=\bar{x} \cdot \bar{a}^{-1} \cdot \bar{y}
$$

as required. 


\subsection{Every definable group in Presburger is abelian- by-finite}

In this subsection we will use some tools developed in [5] by C. Ealy, K. Krupinski and A. Pillay, in order to prove that every definable group in Presburger is abelian-by-finite.

In [5] these authors proved that a rosy dependent group has ICC(see definition 3.2.1), and this implies that the centralizer connected component is a subgroup of finite index. In the first part of this section we recall several results from [5], which we will then use together with the existence of an open box $B_{\bar{a}}$ to prove that $G$ is abelian-by-finite.

At the end of this section we also present an alternative proof that shows that every definable group in Preburger Arithmetic has an abelian subgroup of finite index, which was suggested by A. Onshuus when the proof of $G$ being abelian-by-finite was already written. In fact, his solution is simpler than the first one, so I decided to write both proofs.

\subsubsection{The centralizer connected component and the ICC prop- erty}

Definition 3.2.1. [ICC] Let $\left(G, \cdot, e_{G}\right)$ be a definable group, $G$ has the intersection chain condition (or ICC) if for each $\phi$ there is some $m_{\phi} \in \omega$ such that any chain of intersections of $\phi$-definable subgroups has length at most $m_{\phi}$.

Theorem 3.2.2 (Ealy, Krupinsky, Pillay). Let $\left(G, \cdot, e_{G}\right)$ be a definable group in a dependent rosy theory, then $G$ has $I C C$.

Proof. See Proposition 1.7 [5].

Notice that Presburger Arithmetic is a rosy dependent theory, therefore for every $G$ definable in this theory, $G$ has ICC. Therefore, in the following statements we will assume that we are working in Presburger Arithmetic. 
Remark 3.2.3. Let $G$ be a group and $A \subseteq G$, we define the centralizer of $A$ by

$$
C(A)=\{g \in G \mid g \cdot a=a \cdot g \text { for all } a \in A\}
$$

Observe that $C(A)$ is always a subgroup of $G$. This can be shown as follows:

- For every $a \in A, e_{G} \cdot a=a \cdot e_{G}=a$, then $e_{G} \in G$.

- If $g \in C(A)$, then for all $a \in A, g \cdot a=a \cdot g$, then $a \cdot g^{-1}=g^{-1} \cdot a$. Therefore $g^{-1} \in C(A)$.

- Let $g_{1}, g_{2} \in C(A)$ and $a \in A$. Then $\left(g_{1} \cdot g_{2}\right) \cdot a=g_{1} \cdot\left(g_{2} \cdot a\right)=g_{1} \cdot\left(a \cdot g_{2}\right)=$ $\left(g_{1} \cdot a\right) \cdot g_{2}=\left(a \cdot g_{1}\right) \cdot g_{2}=a \cdot\left(g_{1} \cdot g_{2}\right)$, and we conclude that $g_{1} \cdot g_{2} \in C(A)$.

Corollary 3.2.4. Let $G$ a definable group and $A \subseteq G$, then $C(A)$ is definable.

Proof. Assume that $G$ is defined by the formula $\phi(x, m)$. Notice that for each $a \in A, C(a)$ is defined by $\psi(x, a, m)=\phi(x, m) \wedge((x \cdot a)=(a \cdot x))$, and we have $C(A)=\bigcap_{a \in A} C(a)$. Fix an enumeration $\left\langle a_{\alpha} \mid \alpha<\beta\right\rangle$ of $A$. Then we have the decreasing chain of intersections of $\psi$-definable groups given by $\left\{{ }_{m_{\psi}}\left(a_{\alpha}\right): \alpha<\beta\right\}$. Since $G$ has the ICC property, there is some $m_{\psi} \in \omega$, such that $C(A)=\bigcap_{j=1}^{m_{\psi}} C\left(a_{i_{j}}\right)$. Thus, $C(A)$ is defined by $\bigwedge_{j=1}^{m_{\psi}} \psi\left(x, a_{i_{j}}, m\right)$.

Definition 3.2.5 (Centralizer connected component). Let $G$ be a definable group, we define the centralizer connected component of $G$ as the intersection of all centralizers of finite index. We denote this subset as $H_{G}$.

Corollary 3.2.6. Let $G$ be a definable group, then $H_{G}$ is a definable normal subgroup of finite index.

Proof. Let $\mathcal{A}=\{A \subseteq G \mid C(A)$ has finite index in $G\}$.

1. $H_{G}$ is definable subgroup of $G$ :

By definition, $H_{G}=\bigcap_{A \in \mathcal{A}} C(A)$. However, since $C(A)=\bigcap_{a \in A} C(a)$ for every $A \in$ 
$\mathcal{A}$, we have $H_{G}=\bigcap_{A \in \mathcal{A}} \bigcap_{a \in A} C(a)$. Thus, since $G$ has the ICC property, there are $a_{1}, \ldots, a_{n} \in G$ such that $H_{G}=\bigcap_{i=1}^{n} C\left(a_{i}\right)$. So, since $H_{G}$ is the intersection of finitely many definable subgroups of $G \stackrel{i=1}{H}{ }_{G}$ is itself a definable subgroup of $G$.

2. $H_{G}$ has finite index in $G$ :

We have shown that $H_{G}=\bigcap_{i=1}^{n} C\left(a_{i}\right)$ where for each $i \leq n, a_{i} \in A_{i} \subseteq G$ and $C\left(A_{i}\right)$ has finite index in $G$. Observe that $C\left(A_{i}\right) \leq C\left(a_{i}\right)$, so $C\left(a_{i}\right)$ has also a finite index in $G$ and we have $\left[G: H_{G}\right] \leq \prod_{i=1}^{n}\left[G: C\left(a_{i}\right)\right]<\omega$.

3. $H_{G}$ is a normal subgroup of $G$ : Let $g$ be an arbitrary element of $G$. We will show that $g \cdot H_{G} \cdot g^{-1} \subseteq H_{G}$.

Let $h \in H_{G}$ and let $A$ be an arbitrary subset of $G$ such that $C(A)$ has finite index in $G$. Consider the definable map $f_{g}: C(A) \rightarrow C\left(g^{-1} \cdot A \cdot g\right)$ given by $f_{g}(h)=g^{-1} \cdot h \cdot g$. Observe that $f_{g}$ is injective: if there are two elements $h_{1}, h_{2} \in C(A)$, such that $g \cdot h_{1} \cdot g^{-1}=g \cdot h_{2} \cdot g^{-1}$, then multiplying each side of the equation by $g$ on the the right and $g^{-1}$ on the left we conclude that $h_{1}=h_{2}$. Therefore, by Lemma 2.3.8, $\operatorname{dim}\left(C\left(g^{-1} \cdot A \cdot g\right)\right)=\operatorname{dim}(C(A))=\operatorname{dim}(G)$, and $C\left(g^{-1} \cdot A \cdot g\right)$ has finite index in $G$ by Lemma 2.3.12.

So, $H_{G} \subseteq C\left(g^{-1} \cdot A \cdot g\right)$ and we have $h \in C\left(g^{-1} \cdot A \cdot g\right)$. That is, for every $a \in A$ and $h \in H_{G}$ we have $h \cdot\left(g^{-1} \cdot a \cdot g\right)=\left(g^{-1} \cdot a \cdot g\right) \cdot h$, or equivalently, $\left(g \cdot h \cdot g^{-1}\right) \cdot a=a \cdot\left(g \cdot h \cdot g^{-1}\right)$ for each $a \in A$. This shows that $\left(g \cdot h \cdot g^{-1}\right) \in C(A)$, and since $A$ was chosen arbitrarily with $C(A)$ having finite index in $G$, we have

$$
g^{-1} \cdot h \cdot g \in \bigcap_{A \in \mathcal{A}} C(A)=H_{G} .
$$

We conclude that $g^{-1} \cdot H_{G} \cdot g \subseteq H_{G}$. 
3.2. EVERY DEFINABLE GROUP IN PRESBURGER IS ABELIAN-BY-FINITE 87

\subsection{2 $G$ is abelian-by-finite}

Theorem 3.2.7. Every Presburger-definable group $\left(G, \cdot, e_{G}\right)$ contains a normal abelian subgroup of finite index that is definable. In particular, $G$ is abelian-by-finite.

Proof. Let $\left(G, \cdot, e_{G}\right)$ be a definable group and take a dim-generic element $\bar{a} \in G$. By Lemma 3.1.4, there is $n$-Box $B_{\bar{a}}$ such that for every $\bar{x}, \bar{y} \in B_{\bar{a}}, \bar{x} \cdot \bar{a}^{-1} \cdot \bar{y}=\bar{x}-\bar{a}+\bar{y}$.

Given $\bar{x}, \bar{y} \in G$, define $\bar{x} \star \bar{y}=\bar{x} \cdot \bar{a}^{-1} \cdot \bar{y}$, notice that $\star$ is a group operation which identity is $\bar{a}$. Namely,

- $\star$ is associative: $\bar{z} \star(\bar{x} \star \bar{y})=\bar{z} \star\left(\bar{x} \cdot \bar{a}^{-1} \cdot \bar{y}\right)=\bar{z} \cdot \bar{a}^{-1} \cdot\left(\bar{x} \cdot \bar{a}^{-1} \cdot \bar{y}\right)=\left(\bar{z} \cdot \bar{a}^{-1} \cdot \bar{x}\right) \cdot \bar{a}^{-1} \cdot \bar{y}=$ $(\bar{z} \star \bar{x}) \star \bar{y}$

- $\bar{a}$ is an identity: $\bar{x} \star \bar{a}=\bar{x} \cdot \bar{a}^{-1} \cdot \bar{a}=\bar{x}$,

- Existence of inverse elements: let $\bar{x} \in G$, then take $\bar{a} \cdot \bar{x}^{-1} \cdot \bar{a}$, therefore $\bar{x} \star\left(\bar{a} \cdot \bar{x}^{-1} \cdot \bar{a}\right)=$ $\bar{x} \cdot \bar{a}^{-1} \cdot \bar{a} \cdot \bar{x}^{-1} \cdot \bar{a}=\bar{a}$.

- Claim 1: $\left(G, \cdot, e_{G}\right)$ is definably isomorphic to $(G, \star, \bar{a})$.

Proof: Consider the definable function $f: G \rightarrow G$, defined as $f(\bar{x})=\bar{x} \cdot \bar{a}$. We show that $f$ is an isomorphism. First note that $f$ is injective: if $\bar{x}, \bar{y} \in G$ are such that $f(\bar{x})=f(\bar{y})$, then $\bar{x} \cdot \bar{a}=\bar{y} \cdot \bar{a}$, hence $\bar{x} \cdot \bar{a} \cdot \bar{a}^{-1}=\bar{y} \cdot \bar{a} \cdot \bar{a}^{-1}$, and so therefore $\bar{x}=\bar{y}$. On the other hand, given $\bar{y} \in G$ we have $f\left(\bar{y} \cdot \bar{a}^{-1}\right)=\bar{y}$. This shows that $f$ is surjective.

Finally, $f$ is an homomorphism since for any $\bar{x}, \bar{y} \in G, f(\bar{x} \cdot \bar{y})=(\bar{x} \cdot \bar{y}) \cdot \bar{a}=(\bar{x} \cdot \bar{a}) \cdot$ $\bar{a}^{-1} \cdot(\bar{y} \cdot \bar{a})=f(\bar{x}) \cdot \bar{a}^{-1} \cdot f(\bar{y})=f(\bar{x}) \star f(\bar{y})$. Clearly, $f\left(e_{G}\right)=\bar{a}$.

$\square_{\text {Claim 1. }}$

- Claim 2: $(G, \star, \bar{a})$ is abelian-by-finite.

Proof: Let $H_{G}$ the centralizer connected component of $(G, \star, \bar{a})$. We already know that $H_{G}$ is a normal subgroup of $G$ of finite index. Therefore, it is enough to show that $H_{G}$ is abelian.

Let $\bar{b} \in B_{\bar{a}}$. Since for every $\bar{x} \in B_{\bar{a}}, \bar{b} \star \bar{x}=\bar{b}-\bar{a}+\bar{x}=\bar{x}-\bar{a}+\bar{b}=\bar{x} \star \bar{b}$, then $B_{\bar{a}} \subseteq C(\bar{b})$. This implies that $\operatorname{dim}(C(\bar{b})) \geq \operatorname{dim}(G)$, therefore $C(\bar{b})$ has finite index in $G$. By definition 
of $H_{G}, H_{G} \leq C(\bar{b})$, this means that for all $h \in H_{G}, h \cdot \bar{b}=\bar{b} \cdot h$, then $\bar{b} \in C\left(H_{G}\right)$.

Since $\bar{b}$ is an arbitrary element of $B_{\bar{a}}$, the previous argument actually proves that $B_{\bar{a}} \subseteq C\left(H_{G}\right)$, and since $n=\operatorname{dim}\left(B_{\bar{a}}\right) \leq \operatorname{dim}\left(C\left(H_{G}\right)\right)$, we have that $C\left(H_{G}\right)$ has finite index in $G$. Hence, $H_{G} \leq C\left(H_{G}\right)$, which implies that $H_{G}$ is abelian. We conclude that $(G, \star, \bar{a})$ is abelian-by-finite.

$\square_{\text {Claim 2. }}$

Thus, since $f:\left(G, \cdot, e_{G}\right) \rightarrow(G, \star, \bar{a})$ is an isomorphism, we conclude that $\left(G, \cdot, e_{G}\right)$ is abelian-by-finite, and the normal abelian subgroup of $G$ of finite index is given by $N=f^{-1}\left[\left(H_{G}, \star\right)\right]$.

Corollary 3.2.8. Every Presburger-definable group is amenable. In particular, also definably amenable.

Proof. Let $\left(G, \cdot, e_{G}\right)$ a definable group in Presburger Arithmetic. Since $G$ is abelian-byfinite, it is amenable by Corollary 1.3.7. In particular, $G$ is definably amenable.

The following proof was suggested by A. Onshuus, and we are going to present his argument and latter we state some questions that might be important in this context.

Theorem 3.2.9. Let $\left(G, \cdot, e_{G}\right)$ be a definable group in Presburger. Then there is a definable abelian subgroup of finite index in $G$.

Proof. Let $\left(G, \cdot, e_{G}\right)$ be a definable group in Presburger. By Lemma 3.1.4 there is an $n$-box $B_{\bar{a}}$ around a point $\bar{a} \in G$ such that for every $\bar{x}, \bar{y} \in B_{\bar{a}}$, we have that $\bar{x} \cdot \bar{a}^{-1} \cdot \bar{y}=\bar{x}-\bar{a}+\bar{y}$. Since $\left(G, \cdot, e_{G}\right)$ is definably isomorphic to $(G, \star, \bar{a})$ it is enough to show that there is an abelian subgroup of $(G, \star, \bar{a})$ of finite index.

Define $H=C C\left(B_{\bar{a}}\right)$. Since $B_{\bar{a}}$ is definable (it is a box) then $H$ is also definable. Namely, if $B_{\bar{a}}$ is defined by the formula $\phi(\bar{x}, \bar{b})$ then $H$ is defined by the following formula:

$$
\psi(\bar{y}, \bar{b}):=(\forall \bar{x} \in G(\forall \bar{z}(\phi(\bar{z}, \bar{b}) \rightarrow(\bar{x} \star \bar{z}=\bar{z} \star \bar{x})) \rightarrow \bar{x} \star \bar{y}=\bar{y} \star \bar{x}))
$$


Moreover, since $H$ is the centralizer of the set $C\left(B_{\bar{a}}\right)$, it is also a subgroup of $G$. On the other hand, $B_{\bar{a}} \subseteq C\left(B_{\bar{a}}\right)$ (since $\bar{x} \star \bar{y}=\bar{x}-\bar{a}+\bar{y}=\bar{y}-\bar{a}+\bar{x}=\bar{y} \star \bar{x}$ ), which implies that $H=C C\left(B_{\bar{a}}\right)$ is abelian.

We conclude that $H$ is an abelian definable subgroup of $(G, \star, \bar{a})$, and since it contains the box $B_{\bar{a}}, \operatorname{dim}(H)=\operatorname{dim}(G)$ which implies that $H$ has finite index in $(G, \star, \bar{a})$.

Remark 3.2.10. Notice that by definition $H_{G} \leq C C\left(B_{\bar{a}}\right)$, since $C C\left(B_{\bar{a}}\right)$ is a centralizer of finite index.

Question 3.2.11. Are $H_{G}$ and $C C\left(B_{\bar{a}}\right)$ equal? When can we guarantee that $H_{G}=$ $C C\left(B_{\bar{a}}\right)$ ?

Since $H_{G}$ is a subgroup of finite index of $G$, then $\operatorname{dim}\left(H_{G}\right)=\operatorname{dim}(G)$, so we can take $\bar{a} \in H_{G}$ dim-generic. Moreover, since $H_{G}$ is definable, we can take $B_{\bar{a}} \subseteq H_{G}$.

Claim 1: $H_{G}=C C\left(H_{G}\right)$.

Observe that for every subset $A \subseteq G$, we have that $A \subseteq C C(A)$. Thus, in particular $H \subseteq$ $C C(H)$. On the other hand, since $H=C\left(\left\{a_{1}, \ldots, a_{n}\right\}\right)$ for some elements $a_{1}, \ldots, a_{n} \in$ $\mathcal{M}$, we have that $\left\{a_{1}, \ldots, a_{n}\right\} \subseteq C C\left(\left\{a_{1}, \ldots, a_{n}\right\}\right)$. Therefore $C C C\left(\left\{a_{1}, \ldots, a_{n}\right\}\right) \subseteq$ $C C\left(\left\{a_{1}, \ldots, a_{n}\right\}\right)$, so $C C\left(H_{G}\right) \subseteq H_{G}$.

Claim 2: $H_{G}=C C\left(B_{\bar{a}}\right)$.

Since $B_{\bar{a}} \subseteq H$, we have that $C(H) \subseteq C\left(B_{\bar{a}}\right)$ so $C C\left(B_{\bar{a}}\right) \subseteq C C\left(H_{G}\right)=H_{G}$.

Thus we conclude that $H_{G}=C C\left(B_{\bar{a}}\right)$. 
90CHAPTER 3. A CHARACTERIZATION OF DEFINABLE GROUPS IN PRESBURGER ARIT 


\section{Chapter 4}

\section{Generic sets of definable groups in}

\section{Presburger}

In this section we present a characterization of generic sets of definable bounded groups, in the setting of Presburger Arithmetic. The main motivation is given by the results presented by Peterzil and Pillay in [8] for definably compact groups in o-minimal theories. Their results depend heavily on Alfred Dolich's work [4], on which the author studied the definable functions between two non-algebraic types in the o-minimal context. In this chapter, we study the definable functions between non-algebraic types in the Presburger case, in order to find an analog of Theorem 2.1 in [8], which statement is:

Theorem 4.0.12. Let $X(\bar{a})$ be a definable closed and bounded set and $\mathcal{M}_{0}$ an elementary submodel of $\mathcal{M}$. Then, the following statements are equivalent:

- $\mathcal{F}=\left\{X(\bar{b}) \mid \bar{b} \vDash t p\left(\bar{a} / \mathcal{M}_{0}\right)\right\}$ has the finite intersection property.

- $X(\bar{a}) \cap G\left(\mathcal{M}_{0}\right) \neq \emptyset$.

We start with the following lemma, that establishes a necessary condition for a set to be a generic subset of an arbitrary definable group $G$ in Presburger, and more generally, in any group with a translation-invariant dimension theory. 
Lemma 4.0.13. Let $\left(G, \cdot, e_{G}\right)$ be a definable group in Presburger and assume that $X$ is a definable subset of $G$. If $X$ is left (resp. right) generic, then $\operatorname{dim}(X)=\operatorname{dim}(G)$.

Proof. Let $G$ be a definable group and assume that $X \subseteq G$ is left generic, then there are elements $g_{1}, \ldots, g_{k} \in G$ such that $G=\bigcup_{i=1}^{k} g_{i} \cdot X$, then:

$$
\operatorname{dim}(G)=\max \left\{\operatorname{dim}\left(g_{i} \cdot X\right) \mid 1 \leq i \leq k\right\}
$$

However, observe that the definable function $f_{i}: X \rightarrow g_{i} \cdot X$, defined as $f(x)=g_{i} \cdot x$ is bijective. Thus, for each $i \leq k$ and we have $\operatorname{dim}\left(g_{i} \cdot X\right)=\operatorname{dim}(X)$.

\subsection{Definable functions between two non- algebraic types}

Remark 4.1.1. In the following statements we will be working on a saturated model $\mathcal{M}$ of Presburger Arithmetic, $B$ will be a small set of parameters, and $p\left(x_{1}, \ldots, x_{n}\right)$ is a complete $n$-type over $B$ of dimension $n$. Let $q(y)$ denote a complete non-algebraic 1 - type over $B$, and let $\mathcal{P}, \mathcal{Q}$ be their sets of realizations in $\mathcal{M}^{n}$ and $\mathcal{M}$ respectively. Notice that, by quantifier elimination, $q(y)$ is determined by a $d c l(B)$-cut and by a set of formulas of the form $x \equiv_{n} c$ with $0 \leq c<n$. We will indicate as $\mathcal{Q}^{\prime}$ the set of points that satisfy the same $d c l(B)$-cut implied by the type $q(y)$, i.e., the realizations of the type $q(y) \in S(B)$ restricted to the language $\mathcal{L}^{\prime}=\{<\}$.

Lemma 4.1.2. Let $\mathcal{P}$ and $\mathcal{Q}$ be as above. Let $\bar{a} \in \mathcal{P}$, and $\alpha: \mathcal{P} \rightarrow \mathcal{M}$ a relatively $B$-definable function, such that $\alpha(\bar{a}) \in \mathcal{Q}$. Then $\alpha$ is surjective onto $\mathcal{Q}$.

Proof. If $\beta \in \mathcal{Q}$ then $\operatorname{tp}(\beta / B)=\operatorname{tp}(\alpha(\bar{a}) / B)$, and there is an automorphism $h \in$ $\operatorname{Aut}(\mathcal{M} / B)$ such that $h(\alpha(\bar{a}))=\beta$.

Since $\alpha$ is relatively $B$-definable in $\mathcal{P}$, there is an $\mathcal{L}(B)$-formula $\phi(\bar{x}, y, \bar{b})$ such that 
$\mathcal{M} \models \phi(\bar{x}, y, \bar{b})$ if and only if $y=\alpha(\bar{x})$. Since $h \in \operatorname{Aut}(\mathcal{M} / B)$ we have that $\left(h\left(a_{1}\right), \ldots, h\left(a_{n}\right)\right) \models$ $\operatorname{tp}\left(a_{1}, \ldots, a_{n} / B\right)=p(\bar{x})$ and

$$
\begin{aligned}
\mathcal{M} \models \phi\left(a_{1}, \ldots, a_{n}, \alpha(\bar{a}), \bar{b}\right) & \Leftrightarrow \mathcal{M} \models \phi\left(h\left(a_{1}\right), \ldots, h\left(a_{n}\right), h(\alpha(\bar{a})), \bar{b}\right) \\
& \Leftrightarrow \mathcal{M} \models \phi\left(h\left(a_{1}\right), \ldots, h\left(a_{n}\right), \beta, \bar{b}\right) .
\end{aligned}
$$

Thus, $\beta=\alpha\left(h\left(a_{1}\right), \ldots, h\left(a_{n}\right)\right)$, and we are done.

Lemma 4.1.3. Let $\mathcal{P}$ be the set of realizations of a type $p(\bar{x}) \in S_{n}(B)$ of dimension $n$ and let $\alpha: \mathcal{P} \rightarrow \mathcal{M}$ be a relatively $B$-definable function. Take $\bar{a} \in \mathcal{P}$ arbitrary, and suppose that $q(y)=\operatorname{tp}(\alpha(\bar{a}) / B)$ is a non-algebraic 1-type. Let $\mathcal{Q}^{\prime}$ be as in Remark 4.1.1. Then there are elements $\bar{a}^{1}, \bar{a}^{2}, \in \mathcal{P}$ such that

$$
\alpha\left(\bar{a}^{1}\right)<d c l(\bar{a} B) \cap \mathcal{Q}^{\prime}<\alpha\left(\bar{a}^{2}\right)
$$

Proof. Consider the partial type given by

$$
\Sigma(y)=q(y) \cup\left\{\gamma(\bar{a})<y \mid \gamma \text { is a } B \text {-definable function and } \gamma(\bar{a}) \in \mathcal{Q}^{\prime}\right\}
$$

Observe that by quantifier elimination, $q(y)$ is completely determined by its atomic formulas over $d c l(\bar{a} B)$, and those formulas determine a linear system of congruences together with formulas of the form $b<x<b^{\prime}$ where $b, b^{\prime} \in d c l(B)$.

Let $\Sigma_{0}$ be a finite subset of $\Sigma(y)$. Then we can assume that $\Sigma_{0}(y)$ is a conjunction of the form

$$
\underbrace{\bigwedge_{i=1}^{r}\left(y \equiv_{n_{i}} c_{i}\right)}_{(1)} \wedge \underbrace{\bigwedge_{j=1}^{s}\left(f_{j}\left(b_{j}^{1}\right)<y<g_{j}\left(b_{j}^{2}\right)\right)}_{(2)} \wedge \underbrace{\bigwedge_{k=1}^{t}\left(y>\beta_{k}(\bar{a})\right)}_{(3)}
$$

where for each $i \leq r, 0 \leq c_{i}<n_{i}$ are integers; for each $j \leq s b_{j}^{1}, b_{j}^{2} \in B$ and $f_{j}$ and $g_{j}$ are definable functions, and for all $k \leq t, \beta_{k}(\bar{x})$ is a $B$-definable function. 
By the generalized version of the Chinese reminder theorem we know that the conjunction (1) can be reduced to a single formula of the form $y \equiv_{N} c$.

Additionally, taking $d=\max \left\{f_{i}\left(b_{i}^{1}\right) \mid 1 \leq i \leq s\right\}$ and $d^{\prime}=\min \left\{g_{i}\left(b_{i}^{2}\right) \mid 1 \leq i \leq\right.$ $s\}$, we can reduce (2) to the formula $d<y<d^{\prime}\left(d, d^{\prime} \in \operatorname{dcl}(B)\right)$, and taking $\beta(\bar{a})=$ $\left.\max \left\{\beta_{i}(\bar{a})\right) \mid 1 \leq i \leq k\right\}$, we can reduce (3) to a single formula $y>\beta(\bar{a})$.

By cell decomposition theorem, there is a partition $\mathcal{M}^{n}=\bigcup_{i=1}^{n} D_{i}$ of $\mathcal{M}^{n}$ on $B$-definable $n$-cells, such that for all $i \leq n, D_{i} \cap \operatorname{dom}(\beta)=\emptyset$ or $\beta \uparrow_{D_{i}}$ is a linear $B$-definable function. Since $p(\bar{x})$ is a complete type over $B$, we can assume that there $\mathcal{P} \subseteq D_{i}$ for some $i \leq n$ and the function $\beta(\bar{x})$ has the form

$$
\beta(\bar{x})=\sum_{i=1}^{n} s_{i}\left(\frac{x_{i}-c_{i}}{n_{i}}\right)+\gamma\left(\bar{b}^{\prime}\right), \quad \text { with } \bar{b}^{\prime} \subseteq B
$$

Finally, notice that since $\beta(\bar{a}) \in \mathcal{Q}^{\prime}$, we have $d<\beta(\bar{a})<d^{\prime}$.

Claim: The interval $\left(\beta(\bar{a}), d^{\prime}\right)$ is infinite.

Proof of the Claim: Suppose not. Then there is $j \in \mathbb{N}$ such that $\beta(\bar{a})+j=d^{\prime}$, and we have

$$
\sum_{i=1}^{n} s_{i}\left(\frac{a_{i}-c_{i}}{n_{i}}\right)+\gamma+j=d^{\prime}
$$

from which we can isolate $a_{n}$ as an integer combination of $a_{1}, \ldots, a_{n-1}, d^{\prime}$. Thus, $a_{n} \in$ $\operatorname{dcl}\left(a_{1}, \ldots, a_{n-1}, B\right)$, contradicting that $p(\bar{x}) \in S_{n}(B)$ has dimension $n$.

Hence, since $\left(\beta(\bar{a}), d^{\prime}\right)$ is infinite, we can take a $j \in \mathbb{N}$ such that $\beta(\bar{a})+j \equiv_{N} c$, obtaining $\mathcal{M} \models \Sigma_{0}(\beta(\bar{a})+j)$.

Thus, $\Sigma(y)$ is finitely consistent, and by compactness and saturation there is an element $\alpha_{2} \models \Sigma(y)$. In particular, we have $\alpha_{2} \models q(y)=\operatorname{tp}(\alpha(\bar{a}) / B)$, so by Lemma 4.1.2 there is $\bar{a}_{2} \in \mathcal{P}$ such that $\alpha(\bar{a})=\alpha_{2}$. By construction of the type $\Sigma(y)$, we have that $d c l(\bar{a} B) \cap \mathcal{Q}^{\prime}<$ $\alpha\left(\bar{a}_{2}\right)$. 
We can show similarly that there is $\bar{a}_{1} \in \mathcal{P}$ such that $\alpha\left(\bar{a}_{1}\right)$ realizes the partial type

$$
\Sigma_{1}(y):=q(y) \cup\left\{y<\gamma(\bar{a}) \mid \gamma \text { is a } B \text {-definable function and } \gamma(\bar{a}) \in \mathcal{Q}^{\prime}\right\}
$$

from which we will obtain $\alpha_{1}(\bar{a})<\operatorname{dcl}(\bar{a} B) \cap \mathcal{Q}^{\prime}$. This finishes the proof.

\subsection{The theorem of the finite intersection property and a point}

In [8] Pillay and Peterzil presented a characterization of the definable generic subsets of an abelian and definably compact group in o-minimal theories, using the fact that for definably compact o-minnimal groups, forking is essentially equivalent to finite satisfiability over a small model (cf. Theorem 2.1 in [8]). With this idea in mind, we attempt to find conditions on the definable groups in Presburger similar to "definable compactness" that allow us to recover analogues to results of [8] in Presburger Arithmetic. One such condition might be that the group is bounded. In this section, we collect some lemmas in order to prove a version of Theorem 2.1 [8] in Presburger Arithmetic.

Lemma 4.2.1. Let $\phi(x, \bar{a})$ be a formula defining either 0 -cell or a bounded 1-cell. Assume that $\phi(\mathcal{M}, \bar{a}) \cap \operatorname{dcl}(B)=\emptyset$. Then there is an element $\bar{a}^{\prime} \in \mathcal{M}^{k}$ such that :

$$
\begin{aligned}
& \text { 1. } \operatorname{tp}(\bar{a} / B)=\operatorname{tp}\left(\bar{a}^{\prime} / B\right), \\
& \text { 2. } \phi(\mathcal{M}, \bar{a}) \cap \phi\left(\mathcal{M}, \bar{a}^{\prime}\right)=\emptyset \text {. }
\end{aligned}
$$

Proof. Without loss of generality, we may assume that $\bar{a}$ is a tuple of independent elements over $B$ : if $a_{\ell} \in d c l\left\{a_{i_{1}}, \ldots, a_{i_{k}}\right\}$ then we can replace every appearance of $a_{\ell}$ in $\phi(x ; \bar{a})$ by $f\left(a_{i_{1}}, \ldots, a_{i_{k}}\right)$, for some $B$-definable function $f$. By proceeding in this fashion we obtain a formula $\phi^{\prime}\left(x ; \bar{a}^{\prime}\right)$ (with $\bar{a}^{\prime} \subseteq \bar{a}$ independent) which is equivalent to $\phi(x ; \bar{a})$.

Suppose first that $\phi(x, \bar{a})$ defines a 0 -cell, that is, $\phi(x, \bar{a})$ defines a single point $\left\{x_{0}\right\}$. Then, there is a definable function $\alpha$, such that $x_{0}=\alpha(\bar{a})$. Since $\phi(\mathcal{M}, \bar{a}) \cap d c l(B)=$ 
$\{\alpha(\bar{a})\} \cap d c l(B)=\emptyset$, we have $\alpha(\bar{a}) \notin d c l(B)$. Therefore, the type $q(y)=\operatorname{tp}(\alpha(\bar{a}) / B)$ is non-algebraic, and applying the Lemma 4.1.3, there is an element $\bar{a}^{\prime} \in \mathcal{M}^{k}$ such that $\operatorname{tp}(\bar{a} / B)=\operatorname{tp}\left(\bar{a}^{\prime} / B\right)$ and $\alpha\left(\bar{a}^{\prime}\right)>\operatorname{dcl}(B \bar{a}) \cap \mathcal{Q}^{\prime}$.

Thus, $\alpha\left(\bar{a}^{\prime}\right)>\alpha(\bar{a})$, and in particular, $\phi(\mathcal{M}, \bar{a}) \cap \phi\left(\mathcal{M}, \bar{a}^{\prime}\right)=\{\alpha(\bar{a})\} \cap\left\{\alpha\left(\bar{a}^{\prime}\right)\right\}=\emptyset$.

Suppose now that $\phi(x, \bar{a})$ is a bounded 1-cell, that is, $\phi(x, \bar{a})$ is a equivalent to a set defined by a formula of the form $\left.(\alpha(\bar{a})<x<\beta(\bar{a})) \wedge x \equiv_{N} c\right)$ where $\alpha$ and $\beta$ are definable functions and $0 \leq c<N$ are integers.

Claim 1: $\alpha(\bar{a})$ and $\beta(\bar{a})$ are in the same $d c l(B)$-cut.

Proof: Suppose that $\alpha(\bar{a})$ and $\beta(\bar{a})$ are not in the same $d c l(B)$-cut. Then there is an element $d \in d c l(B)$, such that $\alpha(\bar{a})<d<\beta(\bar{a})$. Since the interval $(\alpha(\bar{a}), \beta(\bar{a}))$ is infinite, either $(\alpha(\bar{a}), d)$ is infinite or $(d, \beta(\alpha))$ is infinite, and so there is an element $m_{0} \in[d-N, d+N] \cap(\alpha(\bar{a}), \beta(\bar{a}))$ satisfying $m_{0} \equiv_{N} c$, that is, $m_{0} \in \phi(\mathcal{M}, \bar{a}) \cap d c l(B)$. A contradiction.

Let $\mathcal{Q}^{\prime}$ be the set of points of $\mathcal{M}$ that are in the same $d c l(B)$-cut as $\alpha(\bar{a})$. By Lemma 4.1.3, there is an element $\bar{a}^{\prime} \in \mathcal{M}^{k}$ such that $\operatorname{tp}(\bar{a} / B)=\operatorname{tp}\left(\bar{a}^{\prime} / B\right)$, and $\alpha\left(\bar{a}^{\prime}\right)>d c l(B \bar{a}) \cap$ $\mathcal{Q}^{\prime}$. In particular, since $\beta(\bar{a}) \in \operatorname{dcl}(B \bar{a}) \cap \mathcal{Q}^{\prime}$, we have $\beta(\bar{a})<\alpha\left(\bar{a}^{\prime}\right)$. Thus,

$$
\phi(\mathcal{M}, \bar{a}) \cap \phi\left(\mathcal{M}, \bar{a}^{\prime}\right) \subseteq(\alpha(\bar{a}), \beta(\bar{a})) \cap\left(\alpha\left(\bar{a}^{\prime}\right), \beta\left(\bar{a}^{\prime}\right)\right)=\emptyset
$$

Lemma 4.2.2. Let $\phi(\bar{x}, \bar{a})$ be a formula defining a bounded $\left(i_{1}, \ldots, i_{n+1}\right)$-cell. Assume that $\phi\left(\mathcal{M}^{n+1}, \bar{a}\right) \cap \operatorname{dcl}(B)=\emptyset$, and let $\pi: \mathcal{M}^{n+1} \rightarrow \mathcal{M}^{n}$ the canonical projection.

Then there is an element $\bar{a}^{\prime} \in \mathcal{M}^{k}$ such that :

1. $\operatorname{tp}(\bar{a} / B)=\operatorname{tp}\left(\bar{a}^{\prime} / B\right)$

2. $\pi\left(\phi\left(\mathcal{M}^{n+1}, \bar{a}\right) \cap \phi\left(\mathcal{M}^{n+1}, \bar{a}^{\prime}\right)\right)$ does not contain any point of $d c l(B)$.

Proof. As in Lemma 4.2.1, we may assume without loss of generality that $\bar{a}$ is a tu- 
ple algebraically independent over $B$. Let $p\left(x_{1}, \ldots, x_{k}\right)=\operatorname{tp}(\bar{a} / B)$ and assume that $\operatorname{dim}(p(\bar{x}))=k$. Let $\phi(\bar{x}, \bar{a})$ be a formula that defines a bounded $\left(i_{1}, \ldots, i_{n+1}\right)$-cell.

We will proceed by induction on $n$. The case $n=0$ follows directly from Lemma 4.2.1.

Suppose now that $\phi\left(x_{1}, \ldots, x_{n+1}, \bar{a}\right)$ is a formula defining a bounded $\left(i_{1}, \ldots, i_{n}, i_{n+1}\right)$ cell. Then $\psi\left(x_{1}, \ldots, x_{n}, \bar{a}\right)=\exists x_{n+1}\left(\phi\left(x_{1}, \ldots, x_{n}, x_{n+1}, \bar{a}\right)\right)$ is a bounded $\left(i_{1}, \ldots, i_{n}\right)$-cell (projection of bounded sets is also bounded). If $\psi\left(\mathcal{M}^{n}, \bar{a}\right) \cap \operatorname{dcl}(B)=\emptyset$. It is enough to take $\bar{a}^{\prime}=\bar{a}$. So, we may assume that $\psi\left(\mathcal{M}^{n}, \bar{a}\right) \cap \operatorname{dcl}(B) \neq \emptyset$.

We can consider two cases:

Case 1: The formula $\phi\left(x_{1}, \ldots, x_{n+1}, \bar{a}\right)$ defines a $\left(i_{1}, \ldots, i_{n}, 0\right)$ cell.

In this case, the set defined by $\phi(\bar{x}, \bar{a})$ has the form

$$
\phi\left(\mathcal{M}^{n+1}, \bar{a}\right)=\left\{\left(x_{1}, \ldots, x_{n}, x_{n+1}\right) \in \mathcal{M}^{n+1} \mid\left(x_{1}, \ldots, x_{n}\right) \in \psi\left(\mathcal{M}^{n}, \bar{a}\right) \wedge x_{n+1}=\alpha(\bar{x})\right\}
$$

where $\alpha$ is an $\bar{a}$-definable function. By cell decomposition we may assume that $\alpha(\bar{x})=\sum_{i=1}^{n} s_{i}\left(\frac{x_{i}-d_{i}}{n_{i}}\right)+\gamma(\bar{a})$, where $\gamma$ is a definable function. Fix an element $c \in \psi\left(\mathcal{M}^{n}, \bar{a}\right) \cap d c l(B)$, and notice that $(c, \alpha(c)) \notin d c l(B)$. Then $\alpha(c) \notin d c l(B)$ which implies that $\gamma(\bar{a}) \notin d c l(B)$. Then $q(y)=\operatorname{tp}(\gamma(\bar{a}) / B)$ is a non-algebraic type and let $\mathcal{Q}^{\prime}$ be the set of points in $\mathcal{M}$ that are in the same $d c l(B)$-cut as $\gamma(\bar{a})$. By Lemma 4.1.3, there is an element $\bar{a}^{\prime}$ which satisfies the same type as $\bar{a}$ over $B$ and $\gamma\left(\bar{a}^{\prime}\right)>\operatorname{dcl}(B \bar{a}) \cap \mathcal{Q}^{\prime}$. In particular $\gamma\left(\bar{a}^{\prime}\right)>\gamma(\bar{a})$, and for each $\bar{x} \in \phi\left(\mathcal{M}^{n}, \bar{a}\right)$ we have

$$
\sum_{i=1}^{n} s_{i}\left(\frac{x_{i}-d_{i}}{n_{i}}\right)+\gamma(\bar{a})<\sum_{i=1}^{n} s_{i}\left(\frac{x_{i}-d_{i}}{n_{i}}\right)+\gamma\left(\bar{a}^{\prime}\right)
$$

This implies that $\phi(\mathcal{M}, \bar{a}) \cap \phi\left(\mathcal{M}, \bar{a}^{\prime}\right)=\emptyset$. Then $\pi\left(\phi(\mathcal{M}, \bar{a}) \cap \phi\left(\mathcal{M}, \bar{a}^{\prime}\right)\right)$ does not contained any point of $d c l(B)$. 


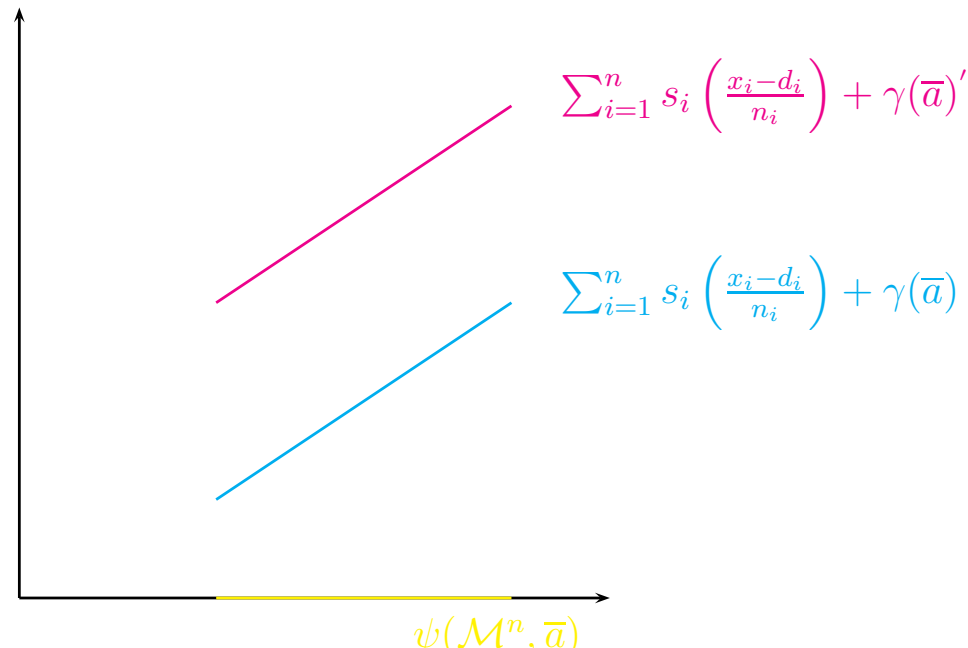

Remark 4.2.3. Observe that $\sum_{i=1}^{n} s_{i}\left(\frac{c_{i}-d_{i}}{n_{i}}\right)+\gamma(\bar{a})$ and $\sum_{i=1}^{n} s_{i}\left(\frac{c_{i}-d_{i}}{n_{i}}\right)+\gamma\left(\bar{a}^{\prime}\right)$ belong to the same $d c l(B)$ cut. Otherwise, there would be an element $d \in d c l(B)$ such that:

$$
\sum_{i=1}^{n} s_{i}\left(\frac{c_{i}-d_{i}}{n_{i}}\right)+\gamma(\bar{a})<d<\sum_{i=1}^{n} s_{i}\left(\frac{c_{i}-d_{i}}{n_{i}}\right)+\gamma\left(\bar{a}^{\prime}\right)
$$

therefore,

$$
\gamma(\bar{a})<d-\sum_{i=1}^{n} s_{i}\left(\frac{c_{i}-d_{i}}{n_{i}}\right)<\gamma\left(\bar{a}^{\prime}\right)
$$

This implies that $\gamma(\bar{a})$ and $\gamma\left(\bar{a}^{\prime}\right)$ belong to different $d c l(B)$ - cuts, which contradicts the fact that $\operatorname{tp}(\bar{a} / B)=\operatorname{tp}\left(\bar{a}^{\prime} / B\right)$.

After this remark, we continue the proof of the Lemma.

Case 2: The formula $\phi\left(x_{1}, \ldots, x_{n+1}, \bar{a}\right)$ defines $a\left(i_{1}, \ldots, i_{n}, 1\right)$ cell.

So, we can assume that $\phi(\bar{x}, \bar{a})$ defines a set of the form

$$
\begin{aligned}
\phi\left(\mathcal{M}^{n+1}, \bar{a}\right) & =\left\{\left(x_{1}, \ldots, x_{n}, x_{n+1}\right) \in \mathcal{M}^{n+1} \mid\left(x_{1}, \ldots, x_{n}\right) \in \psi\left(\mathcal{M}^{n}, \bar{a}\right),\right. \\
& \left.\wedge \alpha\left(x_{1}, \ldots, x_{n}\right) \leq x_{n+1} \leq \beta\left(x_{1}, \ldots, x_{n}\right) \wedge x_{n+1} \equiv_{N} k\right\}
\end{aligned}
$$


where $\alpha$ and $\beta$ are $\bar{a}$-definable functions.By cell decomposition, we can assume that:

$$
\begin{aligned}
& \alpha\left(x_{1}, \ldots, x_{n}\right)=\sum_{i=1}^{n} s_{i}\left(\frac{x_{i}-e_{i}}{n_{i}}\right)+\gamma_{1}(\bar{a}), \\
& \beta\left(x_{1}, \ldots, x_{n}\right)=\sum_{i=1}^{n} p_{i}\left(\frac{x_{i}-d_{i}}{k_{i}}\right)+\gamma_{2}(\bar{a}) .
\end{aligned}
$$

where $\gamma_{1}, \gamma_{2}$ are definable functions and for each $i \leq n, 0 \leq e_{i}<n_{i}$ and $0 \leq d_{i}<k_{i}$ are integers. In order to simplify the notation, we define

$$
f_{1}(\bar{x})=\sum_{i=1}^{n} s_{i}\left(\frac{x_{i}-e_{i}}{n_{i}}\right) \text { and } f_{2}(\bar{x})=\sum_{i=1}^{n} p_{i}\left(\frac{x_{i}-d_{i}}{k_{i}}\right) .
$$

Fix some element element $\bar{c} \in \psi\left(\mathcal{M}^{n}, \bar{a}\right) \cap \operatorname{dcl}(B)$.

- Step 1: $\gamma_{1}(\bar{a}), f_{2}(\bar{c})-f_{1}(\bar{c})+\gamma_{2}(\bar{a}) \notin \operatorname{dcl}(B)$.

Assume by contradiction that $\gamma_{1}(\bar{a}) \in \operatorname{dcl}(B)$, then $f_{1}(\bar{c})+\gamma_{1}(\bar{a}) \in \operatorname{dcl}(B)$. Since the fiber $\left(f_{1}(\bar{c})+\gamma_{1}(\bar{a}), f_{2}(\bar{c})+\gamma_{2}(\bar{a})\right)$ is not empty, then there is some $j \in \mathbb{N}$ such that $f_{1}(\bar{c})+\gamma_{1}(\bar{a})+j \equiv_{N} k$, so $\left(\bar{c}, f_{1}(\bar{c})+\gamma_{1}(\bar{a})+j\right) \in \phi\left(\mathcal{M}^{n+1}, \bar{a}\right) \cap d c l(B) \neq \emptyset$, and this contradicts our hypothesis. In the same way, we can conclude that $\gamma_{2}(\bar{a}) \notin d c l(B)$, which implies that $f_{2}(\bar{c})-f_{1}(\bar{c})+\gamma_{2}(\bar{a}) \notin \operatorname{dcl}(B)$.

-Step 2: $f_{2}(\bar{c})-f_{1}(\bar{c})+\gamma_{2}(\bar{a})$ and $\gamma_{1}(\bar{a})$ are in the same $d c l(B)-c u t$. Suppose that $f_{2}(\bar{c})-f_{1}(\bar{c})+\gamma_{2}(\bar{a})$ and $\gamma_{1}(\bar{a})$ belong to different $d c l(B)$-cuts. Then, there is an element $d \in d c l(B)$ such that $\gamma_{1}(\bar{a})<d<f_{2}(\bar{c})-f_{1}(\bar{c})+\gamma_{2}(\bar{a})$.

Since $\bar{a}$ is algebraically independent over $B$ then $\left(\gamma_{1}(\bar{a}), d\right)$ is infinite. If $\left(\gamma_{1}(\bar{a}), d\right)$ is finite there is an element $z \in \mathbb{Z}$ such that:

$$
\gamma_{1}(\bar{a})+z=d
$$

Since $\gamma_{1}(\bar{a}) \in d c l(\bar{a})$ it is an image of $\bar{a}$ by a linear function. So we can choose $q$ the maxi- 
mum index $i$ such that $a_{i}$ occurs in the equation above. By isolating $a_{q}$ from the equation we conclude that $a_{r} \in d c l\left(B a_{1}, \ldots, a_{q-1}\right)$, but this contradicts the algebraic independence of the tuple $\bar{a}$ over $B$.

Let $r \leq n$ be such that $f_{1}(\bar{c}) \equiv_{N} r$. Then we can take some $j \in \mathbb{N}$ such that $d-j \equiv_{N} k+r$, but this implies $\left(\bar{c}, d-j+f_{1}(\bar{c})\right) \in \phi\left(\mathcal{M}^{n+1}, \bar{a}\right) \cap d c l(B)$, contradicting our hypothesis. Therefore, $\gamma_{1}(\bar{a})$ and $f_{2}(\bar{c})-f_{1}(\bar{c})+\gamma_{2}(\bar{a})$ belong to the same $d c l(B)$-cut.

Moreover, notice that this construction holds for every element $d \in \psi\left(\mathcal{M}^{n}, \bar{a}\right) \cap d c l(B)$. Since $\gamma_{1}(\bar{a})$ does not change, we conclude that for every element $d \in \psi\left(\mathcal{M}^{n}, \bar{a}\right) \cap d c l(B)$, we have that $f_{2}(\bar{d})-f_{1}(\bar{d})+\gamma_{2}(\bar{a})$ is in the same $d c l(B)$-cut of $\gamma_{1}(\bar{a})$.

- Step 3: Finding a tuple $\bar{a}^{\prime}$ such that $t p\left(\bar{a}^{\prime} / B\right)=\operatorname{tp}(\bar{a} / B)$ and $\pi\left(\phi\left(\mathcal{M}^{n+1}, \bar{a}\right) \cap \phi\left(\mathcal{M}^{n+1}, \bar{a}^{\prime}\right)\right)$ does not contain any point of $\operatorname{dcl}(B)$.

Notice that $q(y)=\operatorname{tp}\left(\gamma_{1}(\bar{a}) / B\right)$ is non-algebraic, and for every element $\bar{c} \in \psi\left(\mathcal{M}^{n}, \bar{a}\right) \cap$ $d c l(B)$, we have that $f_{2}(\bar{c})-f_{1}(\bar{c})+\gamma_{2}(\bar{a}) \in d c l(B \bar{a})$ and it belongs to the same $d c l(B)-$ cut as $\gamma_{1}(\bar{a})$. Then applying the Lemma 4.1.3, there is an element $\bar{a}^{\prime} \in \mathcal{M}^{k}$ such that: $\operatorname{tp}(\bar{a} / B)=\operatorname{tp}\left(\bar{a}^{\prime} / B\right)$ and $\gamma_{1}\left(\bar{a}^{\prime}\right)>\operatorname{dcl}(\bar{a} B)$.

Therefore, for each $\bar{c} \in \psi\left(\mathcal{M}^{n}, \bar{a}\right) \cap d c l(B)$, we have $f_{2}(\bar{c})-f_{1}(\bar{c})+\gamma_{2}(\bar{a})<\gamma_{1}\left(\bar{a}^{\prime}\right)$, which implies $f_{2}(\bar{c})+\gamma_{2}(\bar{a})<f_{1}(\bar{c})+\gamma_{1}\left(\bar{a}^{\prime}\right)$. Note that for each $\bar{c} \in \psi\left(\mathcal{M}^{n}, \bar{a}\right) \cap d c l(B)$, the formula $f_{1}(\bar{c})+\gamma_{1}(\bar{x})<f_{2}(\bar{c})+\gamma_{2}(\bar{x})$ is in $\operatorname{tp}(\bar{a} / B)$, so we must have

$$
f_{1}(\bar{c})+\gamma_{1}(\bar{a})<f_{2}(\bar{c})+\gamma_{2}(\bar{a})<f_{1}(\bar{c})+\gamma_{1}\left(\bar{a}^{\prime}\right)<f_{2}(\bar{c})+\gamma_{2}\left(\bar{a}^{\prime}\right)
$$

This implies that $\pi\left(\phi\left(\mathcal{M}^{n+1}, \bar{a}\right) \cap \phi\left(\mathcal{M}^{n+1}, \bar{a}^{\prime}\right)\right)$ does not have any point of $d c l(B)$. 


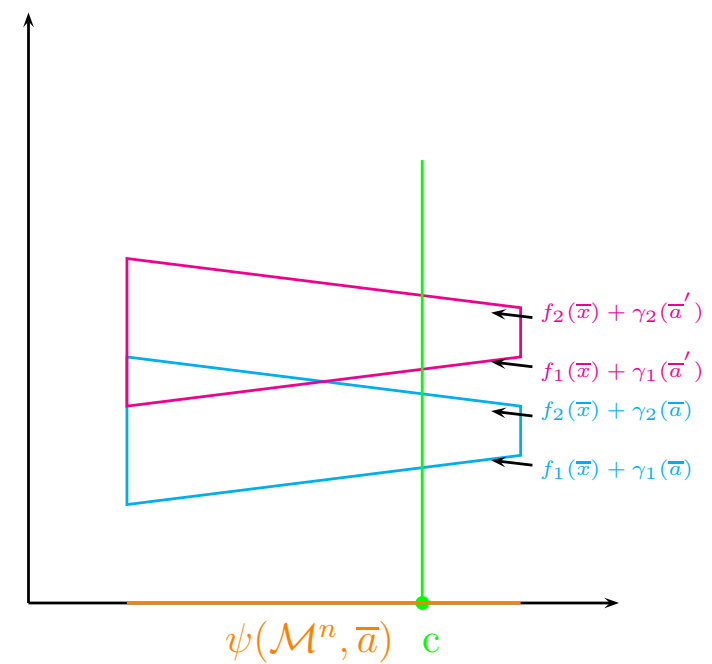

Remark 4.2.4. It is important to notice that for each $\bar{c} \in \psi\left(\mathcal{M}^{n}, \bar{a}\right) \cap d c l(B)$,

$$
f_{1}(\bar{c})+\gamma_{1}(\bar{a})<f_{2}(\bar{c})+\gamma_{2}(\bar{a})<f_{1}(\bar{c})+\gamma_{1}\left(\bar{a}^{\prime}\right)<f_{2}(\bar{c})+\gamma_{2}\left(\bar{a}^{\prime}\right)
$$

are all points in the same $d c l(B)$-cut. Indeed, $f_{1}(\bar{c})+\gamma_{1}(\bar{a})$ and $f_{2}(\bar{c})+\gamma_{2}(\bar{a})$ belong to the same cut because if they were not then there would be an element $d \in \operatorname{dcl}(B)$ such that

$$
f_{1}(\bar{c})+\gamma_{1}(\bar{a})<d<f_{2}(\bar{c})+\gamma_{2}(\bar{a})
$$

So, $\left(d, f_{2}(\bar{c})+\gamma_{2}(\bar{a})\right)$ is infinite, (using the same argument as before) and we can find some $j \in \mathbb{N}$ such that $d+j \equiv_{N} k$. This implies that $\phi\left(\mathcal{M}^{n+1}, \bar{a}\right) \cap d c l(B) \neq \emptyset$, which is a contradiction.

Also, notice that for each for each pair $d_{1}, d_{2} \in d c l(B)$ such that $d_{1}<f_{1}(\bar{c})+\gamma_{1}(\bar{a})<d_{2}$, the formula $d_{1}<f_{1}(\bar{c})+\gamma_{1}(\bar{x})<f_{2}(\bar{c})+\gamma_{2}(\bar{x})<d_{2}$ belongs to $\operatorname{tp}(\bar{a} / B)$, thus it holds also for $\bar{a}^{\prime}$ because $\operatorname{tp}(\bar{a} / B)=\operatorname{tp}\left(\bar{a}^{\prime} / B\right)$.

Lemma 4.2.5. Let $X(\bar{a}) \subseteq \mathcal{M}^{n+1}$ be a bounded definable set, defined over an independent tuple $\bar{a} \in \mathcal{M}^{m}$. Assume that $X(\bar{a}) \cap \operatorname{dcl}(B)=\emptyset$ and let $\pi: \mathcal{M}^{n+1} \rightarrow \mathcal{M}^{n}$ the canonical 
projection on the first $n$ variables. Then there are tuples $\bar{a}_{1}, \ldots, \bar{a}_{k} \models \operatorname{tp}(\bar{a} / B)$, such that $\pi\left(X(\bar{a}) \cap \bigcap_{i=1}^{k} X\left(\bar{a}_{i}\right)\right)$ does not contain any point of $d c l(B)$.

Proof. Let $X(\bar{a}) \subseteq \mathcal{M}^{n+1}$ be a bounded definable set. By cell decomposition, $X(\bar{a})=\bigcup_{j=1}^{k} C_{j}(\bar{a})$, where each $C_{j}(\bar{a})$ is a $\left(i_{1}, \ldots, i_{n+1}\right)$-cell. Since $X(\bar{a}) \cap d c l(B)=\emptyset$, then $C_{j}(\bar{a}) \cap d c l(B)=\emptyset$ for each $j \leq k$, and applying the Lemma 4.2.2 we obtain an element $\bar{a}_{j} \models \operatorname{tp}(\bar{a} / B)$ such that $\pi\left(C_{j}(\bar{a}) \cap C_{j}\left(\bar{a}_{j}\right)\right)$ does not have any point of $d c l(B)$. We will show that these elements $\bar{a}_{1}, \ldots, \bar{a}_{k}$ satisfy the desired condition.

Given an element $\bar{c} \in \operatorname{dcl}(B)$, define $I_{\bar{c}}(\bar{a})=\{x \in \mathcal{M} \mid(\bar{c}, x) \in X(\bar{a})\}$. Observe that $I_{\bar{c}}(\bar{a})$ is a definable set in one variable. Therefore, $I_{\bar{c}}$ is a finite union of 0 and 1 cells $I_{\bar{c}}(\bar{a})<\cdots<I_{\bar{c}}^{l}(\bar{a})$.(In the original decomposition $)$.

Notice that for each $i \leq l$ there is some $s \leq k$, such that $\{\bar{c}\} \times I_{\bar{c}}(\bar{a}) \subset C_{s}(\bar{a})$. Without loss of generality we may assume that $\{c\} \times I_{\bar{c}}^{i}(\bar{a}) \subseteq C_{i}(\bar{a})$.

By the argument stated in the Remarks 4.2.3 and 4.2.4, we know that for any $i \leq l$, we have that $I_{\bar{c}}^{i}(\bar{a})$ is completely contained in some $\operatorname{dcl}(B)$-cut, and we will indicate the points of $\mathcal{M}$ belonging to this cut as $\mathcal{Q}_{i}^{\prime}$.

Step 1: $I_{\bar{c}}(\bar{a}) \cap I_{\bar{c}}^{i}\left(\bar{a}_{i}\right)=\emptyset$.

By the Remarks 4.2.3, 4.2.4 $I_{\bar{c}}^{i}\left(\bar{a}_{i}\right) \subseteq \mathcal{Q}_{i}^{\prime}$ and $I_{\bar{c}}^{i}(\bar{a})$ and $I_{\bar{c}}^{i}\left(\bar{a}_{i}\right)$ are disjoint, and moreover $I_{\bar{c}}^{i}(\bar{a})<I_{\bar{c}}^{i}\left(\bar{a}_{i}\right)$.

Now, fix another element $j \leq l$ such that $j \neq i$. If $\mathcal{Q}_{i}^{\prime}$ and $\mathcal{Q}_{j}^{\prime}$ are different cuts, it is clear that $I_{\bar{c}}^{i}\left(\bar{a}_{i}\right)$ and $I_{\bar{c}}^{j}(\bar{a})$ are disjoint. Therefore, we may assume that $\mathcal{Q}_{i}^{\prime}=\mathcal{Q}_{j}^{\prime}$.

If $I_{\bar{c}}^{j}(\bar{a})<I_{\bar{c}}^{i}(\bar{a})<I_{\bar{c}}^{i}\left(\bar{a}_{i}\right)$ then we are done, so we may assume that $I_{\bar{c}}^{i}(\bar{a})<I_{\bar{c}}^{j}(\bar{a})$. We have the following cases:

1. Case 1: $C_{i}(\bar{a})$ is a $\left(i_{1}, \ldots, i_{n}, 0\right)$ cell

Then $C_{i}(\bar{a})$ is the graph of a definable function $\alpha(\bar{x})=f(\bar{x})+\gamma(\bar{a})$, and moreover $I_{\bar{c}}^{i}(\bar{a})=\{f(\bar{c})+\gamma(\bar{a})\}$. Recall that in the previous construction, we selected $\bar{a}_{i}$ 
4.2. THE THEOREM OF THE FINITE INTERSECTION PROPERTY AND A POINT103

such that $\gamma\left(\bar{a}_{i}\right)>\operatorname{dcl}(B \bar{a}) \cap Q$, where $Q$ is the $\operatorname{dcl}(B)$ cut of $\gamma(\bar{a})$. Let $r(\overline{a c})$ the right endpoint of $I_{\bar{c}}^{j}(\bar{a})$ and notice that it belongs to $\operatorname{dcl}(B \bar{a})$. Additionally, $f(\bar{c})+\gamma(\bar{a})<r(\overline{a c})$, because we assumed that $I_{\bar{c}}^{i}(\bar{a})<I_{\bar{c}}^{j}(\bar{a})$.

Hence, $r(\overline{a c})-f(\bar{c}) \in d c l(B \bar{a})$, so it is enough to show that it belongs to the same $d c l(B)$ - cut as $\gamma(\bar{a})$, because in that case $r(\bar{a} c)<\gamma\left(\bar{a}_{i}\right)+f(c)$, which implies that $I_{\bar{c}}^{i}\left(\bar{a}_{i}\right)$ and $I_{\bar{c}}^{j}(\bar{a})$ are disjoint.

Claim: $r(\overline{a c})-f(\bar{c})$ and $\gamma(\bar{a})$ are in the same $d c l(B)-c u t$

Assume that they are in different cuts, then there is an element $d \in d c l(B)$ such that $\gamma(\bar{a})<d<r(\bar{a} c)-f(\bar{c})$, hence $f(\bar{c})+\gamma(\bar{a})<f(\bar{c})+d<r(\overline{a c})$, but $f(\bar{c})+d \in d c l(B)$ which contradicts the fact that $r(\overline{a c})$ and $f(\bar{c})+\gamma(\bar{a})$ are in the same cut $\mathcal{Q}_{i}^{\prime}$.

2. Case 2: $C_{i}(\bar{a})$ is a $\left(i_{1}, \ldots, i_{n}, 1\right)$ cell

Let $\alpha(\bar{x})=f_{1}(\bar{x})+\gamma_{1}(\bar{a})$ and $\beta(\bar{x})=f_{2}(\bar{x})+\gamma_{2}(\bar{a})$ be the two definable functions which allows to define $C_{i}(\bar{a})$ as the following set

$$
\left\{(\bar{x}, t) \in \mathcal{M}^{n+1} \mid \bar{x} \in \pi\left(C_{i}(\bar{a})\right) \wedge \alpha(\bar{x}) \leq t \leq \beta(\bar{x}) \wedge t \equiv_{N} k\right\}
$$

In the previous construction, we selected $\bar{a}_{i}$ such that $d c l(B \bar{a}) \cap \mathcal{Q}<\gamma_{1}\left(a_{i}\right)$, where $\mathcal{Q}$ is the $d c l(B)$-cut of $\gamma_{1}(\bar{a})$. Again, we will denote as $r(\overline{a c})$ the right endpoint of $I_{\bar{c}}^{j}(\bar{a})$ and we will show that $r(\overline{a c})<\gamma_{1}\left(\bar{a}_{i}\right)+f_{1}(\bar{c})$. Following the same argument presented in the previous claim, we can show that $r(\overline{a c})-f_{1}(\bar{c})$ and $\gamma_{1}(\bar{a})$ are in the same $d c l(B)$-cut, and since $r(\overline{a c})-f_{1}(\bar{c}) \in d c l(B \bar{a})$, then $r(\overline{a c})-f_{1}(\bar{c})<\gamma_{1}\left(\bar{a}_{i}\right)$, which implies that $r(\overline{a c})<f_{1}(\bar{c})+\gamma_{1}\left(a_{i}\right)$. We conclude that $I_{\bar{c}}^{j}(\bar{a}) \cap I_{\bar{c}}^{i}\left(\bar{a}_{i}\right)=\emptyset$.

Moreover, notice that we have shown that if $I_{\bar{c}}^{j}(\bar{a})$ and $I_{\bar{c}}^{i}(\bar{a})$ are contained in the same $d c l(B)$-cut, then $I_{\bar{c}}^{j}(\bar{a})<I_{\bar{c}}^{i}\left(\bar{a}_{i}\right)$.

Step 2: Picking a convenient subset of $\left\{\bar{a}_{1}, \ldots, \bar{a}_{k}\right\}$ for $c$.

Until the moment we have assumed that $I_{\bar{c}}(\bar{a})=I_{\bar{c}}^{1}(\bar{a})<\cdots<I_{\bar{c}}^{l}(\bar{a})$ and that for each $i \leq l$ we have that $\{\bar{c}\} \times I_{\bar{c}}^{i}(\bar{a}) \subseteq C_{i}(\bar{a})$. Now, notice that all these intervals and points 
are contained in some $d c l(B)$-cuts $\mathcal{Q}_{1}^{\prime}<\cdots<\mathcal{Q}_{r}^{\prime}$.

Say that $I_{\bar{c}}(a), \ldots, I_{\bar{c}}(\bar{a})$ are all the intervals and points contained in $\mathcal{Q}_{1}$. Therefore, by the previous argument $I_{\bar{c}}(\bar{a}) \cap I_{\bar{c}}\left(\bar{a}_{1}\right) \cap \mathcal{Q}_{1}^{\prime}=\emptyset$. Indeed, in the Step 1 we proved that for every $j \in \mathbb{N}$ such that $I_{\bar{c}}^{j}(\bar{a}) \subseteq \mathcal{Q}_{1}^{\prime}$, we have that $I_{\bar{c}}^{j}(\bar{a})<I_{\bar{c}}^{1}\left(\bar{a}_{1}\right)$ therefore we have that

$$
I_{\bar{c}}^{1}(\bar{a})<\cdots<I_{\bar{c}}^{s}(\bar{a})<I_{\bar{c}}^{1}\left(\bar{a}_{1}\right)<\cdots<I_{\bar{c}}^{s}\left(\bar{a}_{1}\right)
$$

In particular, $I_{\bar{c}}(\bar{a}) \cap I_{\bar{c}}\left(\bar{a}_{1}\right) \cap \mathcal{Q}_{1}=\emptyset$.

Now, observe that we can repeat this argument for each $d c l(B)$-cut $\mathcal{Q}_{i}^{\prime}$ for $i \leq r$. Indeed, for each $i \leq r$, say that $I_{\bar{a}}^{i_{1}}, \ldots, I_{\bar{a}}^{i_{s_{i}}}$ are all the intervals and points contained in the $\operatorname{dcl}(B)$-cut $\mathcal{Q}_{i}^{\prime}$. Then, we have that $I_{\bar{c}}(\bar{a}) \cap I_{\bar{c}}\left(\bar{a}_{i_{1}}\right) \cap \mathcal{Q}_{i}^{\prime}=\emptyset$.

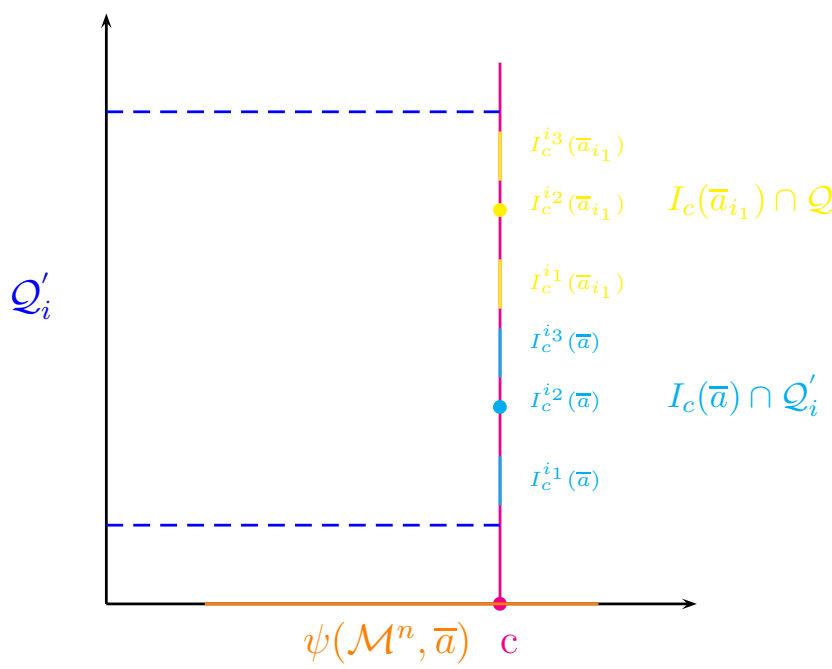

Therefore, notice that if we take the subset $\left\{\bar{a}_{1}, \bar{a}_{2_{1}}, \ldots, \bar{a}_{r_{1}}\right\}$ of $\left\{a_{1}, \ldots, a_{k}\right\}$ then we have that for any $i \leq r, I_{\bar{c}}(\bar{a}) \cap I_{\bar{c}}\left(\bar{a}_{i_{1}}\right) \cap \mathcal{Q}_{i}^{\prime}=\emptyset$. Thus, for every index $i \leq r$ we have that:

$$
I_{\bar{c}}(\bar{a}) \cap I_{\bar{c}}\left(\overline{a_{i_{1}}}\right) \cap \mathcal{Q}_{i}^{\prime}=\emptyset
$$

Step 3: $\pi\left(X(\bar{a}) \cap \bigcap_{i=1}^{k} X\left(\bar{a}_{i}\right)\right)$ does not have any point of $\operatorname{dcl}(B)$.

Assume that there is an element $\bar{c} \in \pi\left(X(\bar{a}) \cap \bigcap_{i=1}^{k} X\left(\bar{a}_{i}\right)\right) \cap \operatorname{dcl}(B)$. Hence, there is 
some $t \in \mathcal{M}$ such that $(\bar{c}, t) \in X(\bar{a}) \cap \bigcap_{i=1}^{k} X\left(\bar{a}_{i}\right)$, then there is some $i$ such that $t \in I_{\bar{c}}(\bar{a}) \subseteq$ $\mathcal{Q}_{i}^{\prime}$. However, for the element $\bar{a}_{i_{1}}$ that have been chosen in the Step 2 for $c$, we have that $I_{\bar{c}}(\bar{a}) \cap I_{\bar{c}}\left(\bar{a}_{i}\right) \cap \mathcal{Q}_{i}^{\prime}=\emptyset$, hence $t \notin I_{\bar{c}}\left(\bar{a}_{i_{1}}\right)$. Then $(c, t) \notin X\left(\bar{a}_{i}\right)$, so we conclude that $\pi\left(X(\bar{a}) \cap \bigcap_{i=1}^{k} X\left(\bar{a}_{i}\right)\right)$ does not have any point of $d c l(B)$.

The following result is a version for bounded definable groups in Presburger Arithmetic of a theorem that was proved by Peterzil and Pillay in [8] in the context of definably compact o-minimal groups.

Theorem 4.2.6. Let $\mathcal{M}_{0}$ be a small elementary submodel of $\mathcal{M}$. Let $X(\bar{a}) \subseteq \mathcal{M}^{n} a$ definable bounded set. Then, the following statements are equivalent:

1. $\left\{X(\bar{b}) \mid \bar{b}=\operatorname{tp}\left(\bar{a} / \mathcal{M}_{0}\right)\right\}$ has the finite intersection property.

2. $X(\bar{a}) \cap \mathcal{M}_{0} \neq \emptyset$.

(which is equivalent to: there is an element $\bar{c} \in \mathcal{M}_{0}$, such that $\bar{c} \in X(\bar{b})$ for every element $\bar{b} \models \operatorname{tp}\left(\bar{a} / \mathcal{M}_{0}\right)$.)

Proof. The direction $(2) \Rightarrow(1)$ is clear. We now show the direction $(1) \Rightarrow(2)$.

Let us assume for a contradiction that $X(\bar{a}) \cap \mathcal{M}_{0}=\emptyset$ and suppose that the family $\mathcal{F}=\left\{X(\bar{b}) \mid \bar{b} \models \operatorname{tp}\left(\bar{a} / \mathcal{M}_{0}\right)\right\}$ has the finite intersection property. We proceed by induction on $n$.

Suppose first that $X(\bar{a})$ is a definable bounded set in one variable. Then $X(\bar{a})$ is a finite union of points or 1-cells $I_{1}(\bar{a})<\cdots<I_{s}(\bar{a})$. Since $X(\bar{a}) \cap \mathcal{M}_{0}=\emptyset$, for each $i \leq s$, $I_{i}(\bar{a})$ is a subset of a $\mathcal{M}_{0}$-cut, which we will denote as $\mathcal{Q}_{i}^{\prime}$. Let $\mathcal{Q}_{1}^{\prime}, \ldots, \mathcal{Q}_{k}^{\prime}$ be the $\mathcal{M}_{0}$-cuts involved in this decomposition with $\mathcal{Q}_{1}^{\prime}<\cdots<\mathcal{Q}_{k}^{\prime}$.

Let $I_{1}(\bar{a})<\cdots<I_{l}(\bar{a})$ be the cells that are in the cut $\mathcal{Q}_{1}^{\prime}$, and by Lemma 4.2.1 there is a tuple $\bar{a}_{1}$ such that to the cell $I_{1}(\bar{a})$. For each $j \leq l$, let $l_{j}(\bar{a})$ and $r_{j}(\bar{a})$ be the left 
and the right endpoints of $I_{j}(\bar{a})$. Notice that $l_{j}(\bar{a}), r_{j}(\bar{a}) \in \operatorname{dcl}\left(\mathcal{M}_{0} \bar{a}\right) \cap \mathcal{Q}_{1}^{\prime}$ since the order in Presburger is discrete. We selected $\bar{a}_{1}$ such that $l_{1}\left(\bar{a}_{1}\right)>d c l\left(\mathcal{M}_{0} \bar{a}\right)$, in particular $r_{l}(\bar{a})<l_{1}\left(\bar{a}_{1}\right)$. Therefore, $I_{1}(\bar{a})<\cdots<I_{l}(\bar{a})<I_{1}\left(\bar{a}_{1}\right)<\cdots<I_{l}\left(\bar{a}_{1}\right)$, and all these intervals are completely contained in $\mathcal{Q}_{1}^{\prime}$. Thus, $X(\bar{a}) \cap X\left(\bar{a}_{1}\right) \cap \mathcal{Q}_{1}^{\prime}=\emptyset$.

Taking $\bar{a}_{2}, \ldots, \bar{a}_{k}$ in a similar way, we have $X(\bar{a}) \cap X\left(\bar{a}_{i}\right) \cap \mathcal{Q}_{i}^{\prime}=\emptyset$ for each $i \leq k$, which implies that $X(\bar{a}) \cap \bigcap_{i=1}^{k} X\left(\bar{a}_{i}\right)=\emptyset$. Therefore, $\{X(\bar{b}) \mid \bar{b} \models \operatorname{tp}(\bar{a} / B)\}$ does not have the finite intersection property.

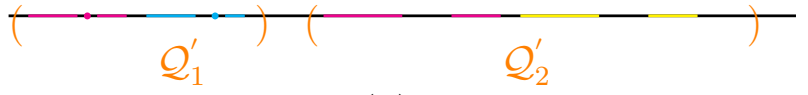

Inductive step: Suppose now that $X(\bar{a})$ is a definable set is a definable set in $n$ variables such that $\mathcal{F}=\left\{X(\bar{b}): \bar{b} \models \operatorname{tp}\left(\bar{a} / \mathcal{M}_{0}\right\}\right.$ has the finite intersection property. If $X(\bar{a}) \cap \mathcal{M}_{0}=\emptyset$, then by Lemma 4.2 .5 , there are $\bar{a}_{1}, \ldots, \bar{a}_{k} \models \operatorname{tp}(\bar{a} / B)$ such that the set $\pi\left(X(\bar{a}) \cap \bigcap_{i=1}^{k} X\left(\bar{a}_{i}\right)\right)$ does not have any point of $\mathcal{M}_{0}$.

Consider the family

$$
\mathcal{F}^{\prime}=\left\{\pi\left(X\left(\bar{b}_{0}\right) \cap \bigcap_{i=1}^{k} X\left(\bar{b}_{i}\right)\right) \mid\left(\bar{b}_{0}, \bar{b}_{1}, \ldots, \bar{b}_{k}\right) \models \operatorname{tp}\left(\left(\bar{a}, \bar{a}_{1}, \ldots, \bar{a}_{k}\right) / \mathcal{M}_{0}\right)\right\}
$$

Claim: $\mathcal{F}^{\prime}$ has the finite intersection property.

Proof: Let $A_{1}, \ldots A_{l} \in \mathcal{F}^{\prime}$, then for each $i \leq l$ there is a tuple $\bar{b}_{0}^{i}, \bar{b}_{1}^{i}, \ldots, \bar{b}_{k}^{i} \models \operatorname{tp}\left(\bar{a}, \bar{a}_{1}, \ldots, \bar{a}_{k} / \mathcal{M}_{0}\right)$, such that $A_{i}=\pi\left(\bigcap_{j=0}^{k} X\left(\bar{b}_{j}^{i}\right)\right)$. Notice that each $\bar{b}_{j}^{i} \models \operatorname{tp}\left(\bar{a} / \mathcal{M}_{0}\right)$, so by the finite intersection property of $\mathcal{F}$ we have that $\bigcap_{i=1}^{l} \bigcap_{j=0}^{k} X\left(\bar{b}_{j}^{i}\right) \neq \emptyset$, and if $\bar{c}$ is an element in such intersection, then $\pi(\bar{c}) \in \pi\left(\bigcap_{i=1}^{l} \bigcap_{j=0}^{k} X\left(\bar{b}_{j}^{i}\right)\right) \subseteq \bigcap_{i=1}^{l}\left[\pi\left(\bigcap_{j=0}^{k} X\left(\bar{b}_{j}^{i}\right)\right)\right]=A_{1} \cap \cdots \cap A_{l}$, as desired.

Therefore, since $\mathcal{F}^{\prime}$ is a uniform family of definable sets in $n$ variables with the finite intersection property, we have by induction hypothesis that there is an element $\bar{c} \in \mathcal{M}_{0}$ such that $\bar{c} \in \pi\left(X(\bar{a}) \cap \bigcap_{i=1}^{k} X\left(\bar{a}_{i}\right)\right)$, which clearly is a contradiction. 


\subsection{Generic sets of definable bounded groups}

In this section we use show that for any bounded group definable in Presburger Arithmetic the collection of the non-generic definable subsets of $G$ form an ideal, following similar ideas to those of Peterzil and Pillay in [8]. This will lead us to show that, in fact, any such group has finitely satisfiable generics, as in Definition 1.2.16.

Definition 4.3.1 (Bounded group). Let $\left(G, \cdot, e_{G}\right)$ be a definable group. We say that $G \subseteq \mathcal{M}^{n}$ is bounded if there is some element $0 \leq \alpha \in \mathcal{M}$ such that for each element $\bar{x}=\left(x_{1}, x_{2}, \ldots, x_{n}\right) \in G$, and for every $1 \leq i \leq n$ we have $-\alpha \leq x_{i} \leq \alpha$

Example 4.3.2. Let $\alpha$ be a non-standard positive element in $\mathcal{M}$. Consider the group $G=[0, \alpha)$ given by the operation $\oplus: G \times G \rightarrow G$ defined as:

$$
x \oplus y:= \begin{cases}x+y & \text { if } x+y<\alpha \\ x+y-\alpha & \text { if } x+y \geq \alpha\end{cases}
$$

Then $(G, \cdot, 0)$ is a definable group and it is bounded by $\alpha$.

Lemma 4.3.3. Let $\mathcal{M}_{0}$ be a small elementary submodel of $\mathcal{M}$. Assume that $G$ is an $\mathcal{M}_{0}$-definable group and $X$ is an $\mathcal{M}_{0}$-definable subset of $G$. Then $X$ is left (right) generic if and only if every right (left) translate of $X$ contains a point in $G\left(\mathcal{M}_{0}\right)$.

Proof. $(\Rightarrow)$ Let $\phi(x, \bar{m})$ and $\psi\left(x, \bar{m}_{2}\right)$ be the formulas defining $G$ and $X$ respectively, with $\bar{m}, \bar{m}_{2} \subseteq \mathcal{M}_{0}$. Assume that $X$ is left generic, then there are $g_{1}, \ldots, g_{k}$ such that $G=\bigcup_{i=1}^{k} g_{i} \cdot X$. We can express this statement with a formula over $\mathcal{M}_{0}$ as follows:

$$
\theta\left(\bar{m}, \bar{m}_{2}\right):=\exists g_{1} \ldots \exists g_{k}\left(\bigwedge_{i=1}^{k} \phi\left(g_{i}, \bar{m}\right) \wedge\left(\forall y\left(\phi(y, \bar{m}) \rightarrow \bigvee_{i=1}^{k} \exists x\left(\psi\left(x, \bar{m}_{2}\right) \wedge y=g_{i} \cdot x\right)\right)\right)\right)
$$

Since $\mathcal{M}_{0}$ is an elementary substructure of $\mathcal{M}$ and $\bar{m}, \bar{m}_{2} \subset \mathcal{M}_{0}$, then $\mathcal{M}_{0} \models \theta\left(\bar{m}, \bar{m}_{2}\right)$, 
108 CHAPTER 4. GENERIC SETS OF DEFINABLE GROUPS IN PRESBURGER

then we can take $g_{1}, \ldots, g_{k} \in G\left(\mathcal{M}_{0}\right)$ such that

$$
\mathcal{M}_{0} \vDash\left(\bigwedge_{i=1}^{k} \phi\left(g_{i}, \bar{m}\right) \wedge\left(\forall y\left(\phi(y, \bar{m}) \rightarrow \bigvee_{i=1}^{k} \exists x\left(\psi\left(x, \bar{m}_{2}\right) \wedge y=g_{i} \cdot x\right)\right)\right)\right)
$$

Since $\mathcal{M}_{0}$ is an elemetary substructure of $\mathcal{M}$, we know that

$$
\mathcal{M} \vDash\left(\bigwedge_{i=1}^{k} \phi\left(g_{i}, \bar{m}\right) \wedge\left(\forall y\left(\phi(y, \bar{m}) \rightarrow \bigvee_{i=1}^{k} \exists x\left(\psi\left(x, \bar{m}_{2}\right) \wedge y=g_{i} \cdot x\right)\right)\right)\right)
$$

Therefore, $G(\mathcal{M})=\bigcup_{i=1}^{k} g_{i} \cdot X$ Let $g \in G$, by hypothesis there is some $i \leq k$ such that $g^{-1} \in g_{i} \cdot X$, it means that there is some $x \in X$ such that $g^{-1}=g_{i} \cdot x$, then $g_{i}^{-1}=x \cdot g \in X \cdot g$. Therefore $(X \cdot g) \cap G\left(\mathcal{M}_{0}\right) \neq \emptyset$.

$(\Leftarrow)$ Assume that $X$ is not left-generic. Consider the type

$$
\Sigma(z)=\{\phi(z, \bar{m})\} \cap\left\{\forall x\left(\psi\left(x, \bar{m}_{2}\right) \rightarrow \neg(z=h \cdot x) \mid h \in \mathcal{M}_{0}\right\}\right.
$$

Since $X$ is not left-generic, the type $\Sigma(z)$ is finitely consistent, thus consistent by compactness. By saturation, there is an element $b \in \mathcal{M}$ such that $\mathcal{M} \models \Sigma(b)$. Thus, for all $h \in \mathcal{M}_{0}, b \notin h \cdot X$, which implies that for each $h \in \mathcal{M}_{0}, h \notin\left(X \cdot g^{-1}\right)$, that is, $\left(X \cdot g^{-1}\right) \cap G\left(\mathcal{M}_{0}\right)=\emptyset$.

Lemma 4.3.4. Let $G$ be a definable group and $X$ be a definable bounded subset of $G$, both defined over $\mathcal{M}_{0}$. Assume that for some $g \in G$ we have that $(X \cdot g) \cap G\left(\mathcal{M}_{0}\right)=\emptyset$. Then there are $g_{1}, \ldots, g_{k} \models \operatorname{tp}\left(g / \mathcal{M}_{0}\right)$ such that $\left(X \cdot g_{1}\right) \cap \cdots \cap\left(X \cdot g_{k}\right)=\emptyset$. In particular, $G \backslash X$ is right generic.

Proof. Since $(X \cdot g) \cap G\left(\mathcal{M}_{0}\right)=\emptyset$, we can apply Lemma 4.2.5, and conclude that the family $\mathcal{F}=\left\{(X \cdot b) \mid b \models \operatorname{tp}\left(g / \mathcal{M}_{0}\right)\right\}$ does not have the finite intersection property. This implies that there are elements $g_{1}, \ldots, g_{k} \models \operatorname{tp}\left(g / \mathcal{M}_{0}\right)$ such that $\left(X \cdot g_{1}\right) \cap \cdots \cap\left(X \cdot g_{k}\right)=\emptyset$. In particular, $G \subseteq\left(\left(X \cdot g_{1}\right) \cap \cdots \cap\left(X \cdot g_{k}\right)\right)^{c}=\bigcup_{i=1}^{k}\left(X \cdot g_{i}\right)^{c}=\bigcup_{i=1}^{k}(G \backslash X) \cdot g_{i}$. 
Corollary 4.3.5. Let $G$ be a definable bounded group and $X$ a definable subset of $G$. If $X$ is not left generic in $G$, then $G \backslash X$ is right generic in $G$.

Proof. Let $\mathcal{M}_{0}$ be an elementary submodel of $\mathcal{M}$ such that both $X$ and $G$ are $\mathcal{M}_{0^{-}}$ definable. Applying the Lemmas 4.3.3 and 4.3.4 we have the desired result.

Corollary 4.3.6. If $G$ is a definable bounded commutative group, then the union of two non-generic sets is also non-generic.

Proof. Let $X_{1}$ and $X_{2}$ be two definable subsets of $G$ and let $X=X_{1} \cup X_{2}$. Let $\mathcal{M}_{0}$ a small elementary submodel such that $X_{1}, X_{2}$ and $G$ are definable over $\mathcal{M}_{0}$. Assume that $X$ is generic, then there are elements $g_{1}, \ldots, g_{k} \in G\left(\mathcal{M}_{0}\right)$ such that

$$
G=\bigcup_{i=1}^{k} g_{i} \cdot X=\bigcup_{i=1}^{k}\left(g_{i} \cdot\left(X_{1} \cup X_{2}\right)\right)=\bigcup_{i=1}^{k}\left(g_{i} \cdot X_{1}\right) \cup \bigcup_{i=1}^{k}\left(g_{i} \cdot X_{2}\right)
$$

Put $A=\bigcup_{i=1}^{k}\left(g_{i} \cdot X_{1}\right)$ and $B=\bigcup_{i=1}^{k}\left(g_{i} \cdot X_{2}\right)$, and notice that $A$ and $B$ are both definable over $\mathcal{M}_{0}$ and bounded, since they are subsets of a bounded set. If $A$ is non-generic, applying the Lemmas 4.3.3 and 4.3.4 we conclude that $G \backslash A$ is generic, which implies that $B$ is generic (because $G \backslash A \subseteq B$ ), and we conclude that $X_{2}$ is generic.

Therefore, if $X$ is generic then at least one of $X_{1}$ or $X_{2}$ is generic.

Let $G$ be a definable group, and define

$$
\mathcal{I}_{G}=\{X \subseteq G \mid X \text { is definable and } X \text { is non-generic in } G\}
$$

By Corollary 4.3.6, if $G$ is a commutative bounded definable group then the set $\mathcal{I}_{G}$ forms an ideal on the algebra of definable subsets of $G$. We will generalize this result to arbitrary bounded definable groups in Presburger Arithmetic, but first we start with the following general lemma. 
Lemma 4.3.7. Let $\left(G, \cdot, e_{G}\right)$ be a definable group, and let $N$ be a normal subgroup of $G$, such that $G / N=\bigcup_{i=1}^{k} s_{i} \cdot N$ and suppose that $\mathcal{I}_{N}$ forms an ideal. Let $X$ be a definable subset of $G$, and for each $i \leq k$ define $X^{i}=X \cap\left(s_{i} \cdot N\right)$. Then,

1. $X$ is left (right) generic in $G$ if and only if the set $\bigcup_{i=1}^{k} s_{i}^{-1} \cdot X^{i}$ is left (right) generic in $N$.

2. If $N$ is abelian, $X$ is left generic in $G$ if and only if $X$ is right generic in $G$.

Proof. 1. $(\Rightarrow)$ Assume that $X$ is left-generic in $G$, therefore there are elements $g_{1}, \ldots, g_{k} \in$ $G$ such that $G=\bigcup_{j=1}^{l}\left(g_{j} \cdot X\right)$ and since $X=\bigcup_{i=1}^{k} X^{i}$, we know that $G=\bigcup_{j=1}^{l} \bigcup_{i=1}^{k}\left(g_{j} \cdot X^{i}\right)$. Observe that $X^{i} \subseteq s_{i} \cdot N$, then for every element $g \in G, g \cdot X^{i} \subseteq s_{j} \cdot N$ for some $j$. Therefore, for $j=1, \ldots, l$ we either have $g_{j} \cdot X^{i} \cap N=\emptyset$ or $g_{j} \cdot X^{i} \subseteq N$.

Let $B$ be the set of pairs of indices $(i, j)$ such that $g_{j} \cdot X^{i} \subseteq N$. Notice that $N=\bigcup_{(i, j) \in B}\left(g_{j} \cdot X^{i}\right)$, and since $\mathcal{I}_{N}$ is an ideal, there is a pair $(i, j) \in B$, such that $g_{j} \cdot X^{i}$ is left-generic in $N$. This implies that $s_{i}^{-1} \cdot X^{i}$ is left-generic in $N$. We conclude that $\bigcup_{i=1}^{k} s_{i}^{-1} \cdot X^{i}$ is left-generic in $N$.

$(\Leftarrow)$ Assume that $\bigcup_{i=1}^{k} s_{i}^{-1} \cdot X^{i}$ is left-generic in $N$. Then there are $h_{1}, \ldots, h_{m} \in N$ such that

$$
N=\bigcup_{j=1}^{m} h_{j} \cdot\left(\bigcup_{i=1}^{k} s_{i}^{-1} X_{i}\right)=\bigcup_{j=1}^{m} \bigcup_{i=1}^{k} h_{j} \cdot s_{i}^{-1}\left(X \cap s_{i} \cdot N\right) \subseteq \bigcup_{j=1}^{m} \bigcup_{i=1}^{k} h_{j} \cdot s_{i}^{-1} X .
$$

So, $G=\bigcup_{r=1}^{k} s_{i} \cdot N \subseteq \bigcup_{i, j, r}\left(s_{r} \cdot h_{j} \cdot s_{i}^{-1}\right) \cdot X$, which shows that $X$ is left-generic in $G$. Notice that the same argument can be done replacing left-generic by right-generic.

2. By (1), $X$ is right-generic in $G$ if and only if $\bigcup_{i=1}^{k} s_{i}^{-1} \cdot X^{i}$ is right-generic in $N$. Since $N$ is abelian, the latter implies that $\bigcup^{k} s_{i}^{-1} \cdot X^{i}$ is also left generic, which by (1) is equivalent to $X$ being left-generic in $\stackrel{i=1}{G}$. 
Lemma 4.3.8. Let $\left(G, \cdot, e_{G}\right)$ be a definable bounded group. Then $I_{G}$ is an ideal in the algebra $\operatorname{Def}(G)$.

Proof. Let $\left(G, \cdot, e_{G}\right)$ be a definable bounded group. By Theorem 3.2.7, we know that $G$ is abelian-by-finite. Moreover, we have shown that there is a definable abelian subgroup $N$ of $G$ of finite index.

Since $N$ is definable and bounded (it is a subset of a bounded set), by Corollary 4.3 .6 we know that $\mathcal{I}_{N}$ is an ideal in $\operatorname{Def}(N)$. Since $N$ has finite index, then there are $s_{1}, \ldots, s_{k} \in$ $G$, such that $G=\bigcup_{i=1}^{k} s_{i} \cdot N$. Let $X_{1}$ and $X_{2}$ be two non-generic definable subsets of $G$ and assume that $X=X_{1} \cup X_{2}$ is generic in $G$. By Lemma 4.3.7, $\bigcup_{i=1}^{k} s_{i}^{-1} \cdot X^{i}$ is generic in $N$. Since $\mathcal{I}_{N}$ is an ideal, there is some $i$ such that $s_{i}^{-1} \cdot X^{i}$ is generic in $N$, and notice that $X^{i}=\left(X_{1}^{i} \cup X_{2}^{i}\right)$, then one of the two sets $s_{i}^{-1} \cdot X_{1}^{i}$ or $s_{i}^{-1} \cdot X_{2}^{i}$ is generic in $N$. Therefore, by Lemma 4.3.7, one of the sets $X_{1}$ or $X_{2}$ is generic in $N$, contradicting our hypothesis. We conclude that $X$ is non-generic, as desired.

Remark 4.3.9. Observe that the condition of bounded is necessary. Indeed, if we consider the abelian definable group $\mathcal{M}=(\mathbb{Z},+, 0)$, note that the definable sets $P=\{x \in \mathcal{M} \mid x \geq$ $0\}$ and $N=\{x \in \mathcal{M} \mid x<0\}$ are both non-generic, but $P \cup N=M$, is clearly generic.

Theorem 4.3.10. Let $G$ be a definable bounded group in the Presburger theory, then it has fsg.

Proof. We show first that there is a small model $\mathcal{M}_{0}$ such that $G$ is definable in $\mathcal{M}_{0}$ and for any definable subset $Y_{\bar{a}}$ of $G$, there is a $\mathcal{M}_{0}$-definable subset $X$ such that $Y \sim_{I} X$. Since $G$ is a Presburger-definable bounded group, it is definably amenable by Corollary 3.2.8, and by Lemma 1.2.24, there are only boundedly many $\sim_{\mathcal{I}}$-classes. Let us consider a set of representatives $\mathcal{C}=\left\{\phi_{\alpha}\left(\bar{x}, \bar{m}_{\alpha}\right) \mid \alpha \in \lambda\right\}$. Thus, by Lowenheim-Skolem theorem, we can find an elementary submodel $\mathcal{M}_{0} \prec \mathcal{M}$ of cardinality $\left|\mathcal{M}_{0}\right| \leq \lambda+|T|$ such that 
$\bigcup_{\alpha \in \lambda} \bar{m}_{\alpha} \subseteq \mathcal{M}_{0}$ and also containing the parameters to define $G$. By construction, $\mathcal{M}_{0}$ satisfies the desired properties.

Claim: For every generic definable subset $Y$ of $G, Y \cap G\left(\mathcal{M}_{0}\right) \neq \emptyset$.

Proof of Claim 1: Let $Y_{\bar{a}}$ be a generic $\bar{a}$-definable subset of $G$, by the Step 1 there is a $\mathcal{M}_{0}$-definable subset $X$ of $G$ such that $Y_{\bar{a}} \sim_{I} X$. Since $Y_{\bar{a}}$ and $X$ are subsets of $G$ they are also bounded, as well as $X \cap Y_{\bar{a}}$ and $Z_{\bar{a}}=X \backslash Y_{\bar{a}}$.

Note that $Z_{\bar{a}}=X \backslash Y_{\bar{a}} \subseteq X \triangle Y_{\bar{a}}$ is non-generic, as well as each $\mathcal{M}_{0}$-conjugate of $Z$ since the property " $G$ cannot be covered by $k$ left-translates of $Z$ " is $\mathcal{M}_{0}$-definable for any $k<\omega$. Therefore, since the non-generic definable subsets of $G$ form an ideal, we conclude that $\bigcup_{i=1}^{k} Z_{\bar{a}_{i}}$ is non-generic.

Suppose $X$ is defined by a formula $\phi(\bar{x}, \bar{m})$, where $\bar{m} \subset \mathcal{M}_{0}$, and assume that $\psi(\bar{x}, \bar{a})$ is the formula defining $Y_{\bar{a}}$. Then, $X \cap Y_{\bar{a}}$ is defined by $\phi(\bar{x}, \bar{m}) \wedge \psi(\bar{x}, \bar{a})$ and $\mathcal{F}=\{\phi(\bar{x}, \bar{m}) \wedge$ $\left.\psi(\bar{x}, \bar{b}) \mid \bar{b} \models \operatorname{tp}\left(\bar{a} / \mathcal{M}_{0}\right)\right\}$ is the family of all $\mathcal{M}_{0^{-}}$conjugates of $X \cap Y_{\bar{a}}$. We now show that $\mathcal{F}$ is finitely consistent.

Assume otherwise. Then there are $\bar{a}_{1}, \ldots, \bar{a}_{k} \models \operatorname{tp}\left(\bar{a} / \mathcal{M}_{0}\right)$ such that $\bigwedge_{i=1}^{k}\left(\phi(\bar{x}, \bar{m}) \wedge \psi\left(\bar{x}, \bar{a}_{i}\right)\right)$ is inconsistent. Let us use $Y_{a_{i}}$ denote the set defined by $\psi\left(\bar{x}, \bar{a}_{i}\right)$. Then we have $X \cap \bigcap_{i=1}^{k} Y_{\bar{a}_{i}}=\emptyset$, that is, $X \subseteq\left(\bigcap_{i=1}^{k} Y_{\bar{a}_{i}}\right)^{c}=\bigcup_{i=1}^{k}\left(Y_{\bar{a}_{i}}\right)^{c}$. By putting $Z_{\bar{a}_{i}}=X \backslash Y_{\bar{a}_{i}}$ we conclude that $X \subseteq \bigcup_{i=1}^{k} Z_{\bar{a}_{i}}$. On the other hand, since $Y_{\bar{a}}$ is generic and $Y_{\bar{a}}=\left(Y_{\bar{a}} \backslash X\right) \cup\left(Y_{\bar{a}} \cap X\right)$ then by Lemma 4.3.8 one of the sets $\left(Y_{\bar{a}} \backslash X\right)$ or $\left(Y_{\bar{a}} \cap X\right)$ must be generic, but it cannot be $Y_{\bar{a}} \backslash X$ because $Y_{\bar{a}} \backslash X \subseteq X \triangle Y_{\bar{a}}$ and $X \sim_{I} Y_{\bar{a}}$. Thus, $Y_{\bar{a}} \cap X$ is generic and also $X$ is generic, which contradicts the fact that $\bigcup_{i=1}^{k} Z_{\bar{a}_{i}}$ is non-generic.

This contradiction shows that the family $\mathcal{F}$ has the finite intersection property, and by Theorem 4.2.6, there is an element $\bar{c} \in\left(X \cap Y_{\bar{a}}\right) \cap G\left(\mathcal{M}_{0}\right)$, in particular it belongs to $Y \cap G\left(\mathcal{M}_{0}\right)$ 
$\mathcal{A}=\{\neg(\phi(\bar{x}, \bar{a})) \mid \phi(\bar{x}, \bar{a})$ defines a non-generic subset of $G\}$.

Since $\mathcal{I}_{G}$ is an ideal and $G$ is generic, then for any definable subset $X$ of $G$ either $X$ is generic or $G \backslash X$ is generic. This implies in particular that the finite intersection of complements of non-generic sets is generic: if $Y_{1}, \ldots, Y_{n}$ are non-generic sets, then $\left(Y_{1} \cup \cdots \cup Y_{n}\right)$ is also non-generic and we have that $G \backslash\left(Y_{1} \cup \cdots \cup Y_{n}\right)=\left(Y_{1}^{c} \cap \cdots Y_{n}^{c}\right)$ is generic.

Thus, the set $\mathcal{A}$ is finitely consistent, and we can take $p$ to be any completion of $\mathcal{A}$ to a global type.

Let $Y \in p$ and $g \in G, Y$ is generic by construction, and this implies that $g \cdot Y$ is also generic. Therefore, by the claim above, $g \cdot Y \cap G\left(\mathcal{M}_{0}\right) \neq \emptyset$ for every $g \in G$. We conclude that $G$ has the fsg property. 
114 CHAPTER 4. GENERIC SETS OF DEFINABLE GROUPS IN PRESBURGER 


\section{Chapter 5}

\section{Ellis semigroup conjecture}

Our main purpose is to prove the Ellis semigroup conjecture (stated by Newelski) for bounded definable groups in Presburger Arithmetic. In the first part of this chapter we recall several definitions and relevant facts in topological dynamics. We refer the reader to [1] or [6] for a detailed reading in this topic. On the second part of this section, we include the proof due to A. Pillay in [9], in which this author showed that the Ellis semigroup conjecture holds for any definable group $G$ with the $f s g$ property.

\subsection{Topological dynamics}

Definition 5.1.1 (G-flow). We will say that $(G, X, \pi)$ is a $G$-flow if:

- $G$ is a topological group,

- $X$ is a compact Hausdorff topological space,

- $\pi: G \times X \rightarrow X$ is an action of $G$ over $X$. And for all $g \in G, \pi_{g}: X \rightarrow X$ is an homeomorphism, where $\pi_{g}(x)=g \cdot x$.

Definition 5.1.2 (Subflow). Let $(G, X, \pi)$ be a $G$-flow. If $Y \subsetneq X$ is a nonempty closed and $G$-invariant subset of $X$, then we say that $(G, Y, \pi)$ is a subflow of $(G, X, \pi)$. 
Definition 5.1.3 (Minimal flow). Let $(G, X, \pi)$ be a $G$-flow, we say that it is minimal if it does not contained any subflow.

Remark 5.1.4. Let $(G, X, \pi)$ a $G$-flow, we denote as $G \cdot x=\left\{\pi_{g}(x) \mid g \in G\right\}$. This set is called as the orbit of $x$.

Lemma 5.1.5. Let $(G, X, \pi)$ be a $G$-flow. Then it is minimal if and only if for any $x \in X$ we have $\overline{G \cdot x}=X$.

Proof. $(\Rightarrow)$ Assume that there is an element $x \in X$, such that $\overline{G \cdot x} \subsetneq X$. Since $G \cdot x$ is $G$ invariant, then $\overline{G \cdot x}$ is also $G$-invariant, and it is closed and nonempty because $x \in \overline{G \cdot x}$. Then $(G, \overline{G \cdot x}, \pi)$ is a subflow of $(G, X, \pi)$ and $(G, X, \pi)$ is not minimal.

$(\Leftarrow)$ Suppose that $(G, X, \pi)$ is not minimal. Then, there is a nonempty closed set $Y \subsetneq X$ which is $G$-invariant. Take $y \in Y$, since it is $G$-invariant $G \cdot y \subset Y$, then $\overline{G \cdot y} \subset Y$, because $Y$ is closed. Therefore, $\overline{G \cdot y} \subsetneq X$.

Lemma 5.1.6. Let $(G, X, \pi)$ a $G$-flow. Then, there is a subflow $(G, Y, \pi)$ which is minimal.

Proof. Let $\mathcal{S}=\{C \subsetneq X \mid C$ is $G$-invariant, closed and non empty $\}$, this set is non empty because $X \in \mathcal{S}$. Consider the partial order over $\mathcal{S}$ defined as $C_{1} \leq C_{2}$ if and only if $C_{2} \subseteq C_{1}$, and let $\mathcal{C}=\left\{C_{\beta} \mid \beta \in \alpha\right\}$ a chain in $\mathcal{C}$. Let $C=\bigcap_{\beta \in \alpha} C_{\beta}$, which is closed because it is the intersection of closed sets. Also, it is non empty since $\mathcal{C}$ has the finite intersection property and $X$ is compact. On the other hand, let $g \in G$ and $c \in C$, since for any $\beta \in \alpha$, $C_{\beta}$ is $G$-invariant then for every $\beta \in \alpha$ we have $g \cdot c \in C_{\beta}$, therefore $g \cdot c \in C$. Then, $C$ is also $G$-invariant, so $C$ is an upper bound of $\mathcal{C}$. Therefore applying Zorn's lemma we can find a maximal set $Y$ in $(\mathcal{S},<)$.

Since $Y \in \mathcal{S},(G, Y, \pi)$ is a $G$-flow and it is minimal by the maximality of $Y$.

Definition 5.1.7 (Isomorphism between $G$-flows). Let $(G, X, \pi)$ and $(G, Y, \phi)$ be two $G$-flows. Let $f: X \rightarrow Y$ be a continuous function from $X$ to $Y$, such that and for every 
$g \in G$ and $x \in X$ we have that:

$$
f(\pi(g, x))=\phi(g, f(x))
$$

In this case we say that $f$ is a $G$-flow homomorphism. Moreover if $f$ is a bijection and its inverse is continuous, then we say that $f$ is an isomorphism of $G$-flows.

Lemma 5.1.8. Let $(G, X, \pi)$ and $(G, Y, \phi)$ be two $G$-flows and let $f: X \rightarrow Y$ be a $G$-flow homomorphism. Let $(G, Z)$ be a minimal subflow of $(G, X)$, then $(G, f(Z))$ is a minimal subflow of $(G, Y)$.

Proof. Step 1: $(G, f(Z))$ is a $G$-flow

In the first place, notice that $f(Z)$ is non-empty since $Z$ is not empty. Also, $f(Z)$ is closed, because $Z$ is a closed set of a compact space, then it is also compact, and since $f$ is continuous $f(Z)$ is a compact subset of a Hausdorff space, therefore it is closed. Therefore, it is enough to show that it is $G$-invariant. Let $g \in G$ and $y \in f(Z)$. Then, there is an element $z \in Z$, such that $f(z)=y$, therefore $\phi(g, y)=\phi(g, f(z))=f(\pi(g, z))$. And since $Z$ is $G$-invariant, $\pi(g, z) \in Z$, so $f(\pi(g, z)) \in f(Z)$, thus we conclude that $\phi(g, y) \in f(Z)$, which implies that $f(Z)$ is $G$-invariant.

Step 2: $(G, f(Z))$ is minimal

Let $y \in f(Z)$, we will show that $\overline{G \cdot y}=f(Z)$. Fix an element $z \in Z$, such that $f(z)=y$. Then, since for every element $g \in G, f(\pi(g, z))=\phi(g, f(y))$, then $f(G \cdot z) \subseteq G \cdot f(y)$, and by the continuity of $f, f(\overline{G \cdot z}) \subseteq \overline{f(G \cdot z)} \subseteq \overline{G \cdot y}$. Moreover, since $Z$ is minimal, $\overline{G \cdot z}=Z$, therefore $f(Z) \subseteq \overline{G \cdot y}$, therefore $f(Z)=\overline{G \cdot y}$.

\subsection{The Ellis Group}

Let $(G, X)$ be a $G$-flow, we can consider the space $X^{X}=\{f: X \rightarrow X \mid f$ is a function $\}$, with the product topology. $X^{X}$ is a Hausdorff compact space, since it is a product of a 
Hausdorff compact space. Also, notice that we can view the action of $G$ on $X$ as a subset of $X^{X}$,because for every $g \in G, \pi_{g}: X \rightarrow X$ is a function. Additionally, observe that $X^{X}$ has a natural semigroup operation given by the composition.

Definition 5.2.1 (Ellis Semigroup). Let $(G, X, \pi)$ a $G$ - flow. Its enveloping semigroup is the structure $(E(X), \circ)$, where $E(X)=\overline{\left\{\pi_{g} \mid g \in G\right\}}$ and $\circ$ is the composition is known as the Ellis semigroup of $(G, X, \pi)$.

Remark 5.2.2. $(E(X), \circ)$ is a subsemigroup of $\left(X^{X}, \circ\right)$ :

Let $f, l \in E(X)$ and consider $U_{1}, \ldots, U_{N}$ open sets of $X$ and $x_{1}, \ldots, x_{n} \in X$, such that $\bigwedge_{i=1}^{n}\left(l \circ f\left(x_{i}\right) \in U_{i}\right)$. Since $\bigwedge_{i=1}^{n}\left(l\left(f\left(x_{i}\right)\right) \in U_{i}\right)$ and $l \in \overline{\left\{\pi_{g} \mid g \in G\right\}}$, then there is an element $g \in G$, such that $\bigwedge_{i=1}^{n}\left(\pi_{g}\left(f\left(x_{i}\right)\right) \in U_{i}\right)$. This implies that $\bigwedge_{i=1}^{n}\left(f\left(x_{i}\right) \in \pi_{g}^{-1}\left(U_{i}\right)\right)$, and $\pi_{g}^{-1}\left(U_{i}\right)$ is open since $\pi_{g}$ is an homeomorphism. Then, there is another element $h \in G$, such that $\bigwedge_{i=1}^{n}\left(\pi_{h}\left(x_{i}\right) \in \pi_{g}^{-1}\left(U_{i}\right)\right)$, therefore $\bigwedge_{i=1}^{n}\left(\pi_{g} \circ \pi_{h}\left(x_{i}\right) \in U_{i}\right)$ which implies that $\bigwedge_{i=1}^{n}\left(\pi_{g \cdot h}\left(x_{i}\right) \in U_{i}\right)$. Hence, we conclude that $g \circ f \in E(X)$.

Lemma 5.2.3. $(G, E(X), \phi)$ is a $G$-flow, where $\phi: G \times E(X) \rightarrow E(X)$ is defined as $\phi(g, f)=\pi_{g} \circ f$.

Proof. - Step 1: E $(X)$ is a compact Hausdorff space $E(X)$ is Hausdorff because it is a subspace of a Hausdorff space. And it is compact because it is a closed subset of the compact space $X^{X}$.

- Step 2: For each $g \in G, \phi_{g}: E(X) \rightarrow E(X)$ is an homeomorphism $\phi_{g}$ is bijective:

injective: let $f, h \in E(X)$ and assume that they are different functions. Then, there is an element $x \in X$, such that $f(x) \neq h(x)$, so there are two open sets $U$ and $V$ in $X$ such that:

$$
\text { - } f(x) \in U \text { and } h(x) \in V \text {, }
$$


$-U$ and $V$ are disjoint.

Since, $\pi_{g}$ is an homeomorphism, $\pi_{g}(U)$, and $\pi_{g}(V)$ are also open. Actually, they are disjoint, because if there is an element $z \in \pi_{g}(U) \cap \pi_{g}(V)$, then there are $u \in U$ and $v \in V$ such that $\pi_{g}(u)=\pi_{g}(v)=z$, but since $\pi_{g}$ is injective $u=v$, which implies that $U \cap V \neq \emptyset$. Then, since $\pi_{g} \circ f(x) \in \pi_{g}(U)$ and $\pi_{g} \circ h(x) \in \pi_{g}(V)$, and $\pi_{g}(U) \cap \pi_{g}(V)=\emptyset$, then $\pi_{g} \circ f(x) \neq \pi_{g} \circ h(x)$, therefore $\pi_{g} \circ f \neq \pi_{g} \circ h$.

surjective: let $f \in E(X)$, and consider $h=\pi_{g^{-1}} \circ f$, then for all $x \in X, \phi_{g}(h(x))=$ $\pi_{g} \circ \pi_{g^{-1}} \circ f(x)=f(x)$, then $\phi_{g}$ is sujective.

- $\phi_{g}$ is continuous and open:

Let $U_{1}, \ldots, U_{n}$ be open sets of $X$ and $x_{1}, \ldots, x_{n} \in X$. Consider the basic open set of $E(X)$,

$$
\mathcal{O}=\left\{f \in E(X) \mid \bigwedge_{i=1}^{n}\left(f\left(x_{i}\right) \in U_{i}\right)\right\}
$$

Then,

$$
\begin{aligned}
\phi_{g}^{-1}(\mathcal{O}) & =\left\{f \in E(X) \mid \pi_{g} \circ f \in \mathcal{O}\right\} \\
& =\left\{f \in E(X) \mid \bigwedge_{i=1}^{n}\left(\pi_{g}\left(f\left(x_{i}\right)\right) \in U_{i}\right)\right\} \\
& \left.=\left\{f \in E(X) \mid \bigwedge_{i=1}^{n}\left(f\left(x_{i}\right)\right) \in \pi_{g}^{-1}\left(U_{i}\right)\right)\right\}=\mathcal{U} .
\end{aligned}
$$

Since $\pi_{g}$ is an homeomorphism, then $\pi_{g}^{-1}\left(U_{i}\right)$ is open in $X$ for each $1 \leq i \leq n$, which implies that $\mathcal{U}$ is also open.

Similarly, we can show that $\phi_{g}$ is open.

Lemma 5.2.4. Let $E(X)$ be the Ellis semigroup of a $G$ flow $(G, X, \pi)$. Then for every element $p \in E(X)$, the function $S_{p}: E(X) \rightarrow E(X)$ defined as $S_{p}(f)=f \circ p$ is continuous 
and closed.

Proof. - $\quad S_{p}$ is continuous: let $U_{1}, \ldots, U_{n}$ be open sets in $X$ and $x_{1}, \ldots, x_{n} \in X$. Let $\mathcal{O}$ be the following basic open set

$$
\mathcal{O}=\left\{f \in E(X) \mid \bigwedge_{i=1}^{n}\left(f\left(x_{i}\right) \in U_{i}\right)\right\}
$$

then

$$
\begin{aligned}
S_{p}^{-1}(\mathcal{O}) & =\{f \in E(X) \mid f \circ p \in \mathcal{O}\} \\
& =\left\{f \in E(X) \mid \bigwedge_{i=1}^{n}\left(f \circ p\left(x_{i}\right) \in U_{i}\right)\right\}=\mathcal{U}
\end{aligned}
$$

Now notice that $\mathcal{U}$ is also open, because if we take $y_{1}=p\left(x_{1}\right), \ldots, y_{n}=p\left(x_{n}\right)$, then $\mathcal{U}=\left\{f \in E(X) \mid \bigwedge_{i=1}^{n} f\left(y_{i}\right) \in U_{i}\right\}$, which is a basic open set of $E(X)$, therefore $S_{p}$ is continuous.

- $S_{p}$ is closed: let $C \subseteq E(X)$ be a closed set, then it is also compact. Since $S_{p}$ is continuous, $S_{p}(C)$ is compact, then it is closed.

Fact 5.2.5. For every element $p \in E(X)$, we have that $\overline{G \cdot p}=E(X) \circ p$.

Proof. Observe that

$$
E(X) \circ p=S_{p}(E(X))=S_{p}\left(\overline{\left\{\pi_{g} \mid g \in G\right\}}\right)=\overline{S_{p}\left(\left\{\pi_{g} \mid g \in G\right\}\right)}=\overline{G \cdot p} .
$$

Notice that $S_{p}\left(\overline{\left\{\pi_{g} \mid g \in G\right\}}\right)=\overline{S_{p}\left(\left\{\pi_{g} \mid g \in G\right\}\right)}$, because $S_{p}$ is continuous and closed.

Definition 5.2.6. Let $(S, \star)$ be a semigroup. And let $I \subseteq S$, we say that it is a left ideal if $S \star I \subset I$. Moreover, we say that $I$ is minimal, if for every $F \subset I$ left ideal then $F=I$. 
Lemma 5.2.7. Let $(G, X, \pi)$ a $G$-flow. Then, the minimal left ideals of $(E(X), \circ)$ coincide with the minimal flows of $(G, E(X), \phi)$.

Proof. $(\Leftarrow)$ Let $(G, Y, \pi)$ be a minimal flow of $(G, E(X), \phi)$ and let $p \in Y$. Since $Y$ is invariant, then $G \cdot p=\left\{\pi_{g} \circ p \mid g \in G\right\} \subseteq Y$. Therefore, $\overline{G \cdot p} \subset \bar{Y}=Y$. Now observe that, $E(X) \circ p=\{f \circ p \mid f \in E(X)\}=S_{p}\left(\overline{\left\{\pi_{g} \mid g \in G\right\}}\right) \subseteq \overline{S_{p}\left(\left\{\pi_{g} \mid g \in G\right\}\right)}=\overline{G \cdot p} \subset Y$. Then $Y$ is a left ideal.

Now, we just need to show that $Y$ is a minimal ideal. So let $I$ be a non empty left ideal, such that $I \subseteq Y$. Take an element $p \in I$, therefore $\overline{G \cdot p}=E(X) \circ p \subset E(X) \circ I \subset I$. However, since $Y$ is minimal, then $Y=\overline{G \cdot p}$, then $Y \subset I$, which implies that $Y=I$, then $Y$ is a minimal left ideal.

$(\Rightarrow)$ Let $I$ a minimal left ideal of $(E(X), \circ)$. First, we will show that $I$ is a non empty closed $G$-invariant set. In order to show that it is a minimal flow we will prove that for every element $p \in I$ we have that $\overline{G \cdot p}=I$.

$I$ is G-invariant: take an element $p \in I$, then $G \cdot p=\left\{\pi_{g} \circ p \mid g \in G\right\} \subseteq E(X) \circ I \subset I$, this implies that $I$ is $G$-invariant.

$I$ is closed and minimal: observe that $E(X) \circ p=\overline{G \cdot p}$ is a left ideal and is contained in $I$, then by minimality of $I$, we have $I=\overline{G \cdot p}$. Then $I$ is closed, and since this statement holds for every $p \in I, I$ is a minimal flow.

Definition 5.2.8 (Idempotent). Let $(S, \star)$ be a semigroup. We say that $u \in S$ is idempotent if $u \star u=u$.

Lemma 5.2.9. Let $(G, X, \pi)$ be a $G$-flow. And let $E(X)$ be its enveloping semigroup and I a minimal left ideal, then the following conditions hold:

1. The set $J$ of idempotents of $I$ is not empty.

2. If $v \in J$ and $p \in I$, then $p \circ v=p$.

3. $(v \circ I, \circ, v)$ is a group. 
Proof. $\quad 1$. Let $\mathcal{S}$ be the collection of all non-empty closed subsets $S$ of $I$ such that $S^{2} \subseteq S$. Notice that $\mathcal{S} \neq \emptyset$, because $I \in \mathcal{S}$. Observe that $\mathcal{S}$ can be ordered as follows: $S_{1} \leq S_{2}$ if and only if $S_{2} \subseteq S_{1}$. Let $\left\{C_{\beta}\right\}_{\beta \in \alpha}$ be a chain of $(\mathcal{S}, \leq)$, it means that

$$
C_{1} \supseteq C_{2} \supseteq C_{3} \supseteq \cdots \supseteq C_{\beta} \supseteq C_{\beta+1} \supseteq \cdots
$$

Consider $C=\bigcap_{\beta \in \alpha} C_{\beta}$, since $I$ is compact $C \neq \emptyset$ and it is closed because it is the intersection of closed sets. Additionally, observe that $C^{2} \subseteq C$, because if $c, d \in C$, then $c, d \in C_{\beta}$ for any $\beta \in \alpha$, and since $C_{\beta}^{2} \subseteq C_{\beta}$, therefore $\operatorname{cod} \in C_{\beta}$, thus $c \circ d \in C$. By Zorn's Lemma, there is a maximal element $S$ in this order. Fix an element $x \in S$ and consider $S \circ x=S_{x}(S)$, which is closed because it is the image of a closed set under a continuous function (by Lemma 5.2.4). Also, observe that $S \circ x \subseteq S^{2} \subseteq S$, and $(S \circ x) \circ(S \circ x) \subseteq(S \circ S) \circ(S \circ x) \subseteq S \circ x$. Therefore, by maximality of $S, S \circ x=S$, then there is an element $u \in S$, such that $u \circ x=x$. Notice that $u \in W=S_{x}^{-1}(\{x\}) \cap S$, which is closed and is contained in $S$. And, for every two elements $u, v \in W, u \circ x=v \circ x=x$, then $(u \circ v) \circ x=u \circ(v \circ x)=u \circ x=x$. Clearly $u \circ v \in S^{2} \subset S$, then $u \circ v \in W$, this proves that $W^{2} \subseteq W$. Therefore, by maximality of $S$, we have that $W=S$. And this implies that $x \in W$, so $x \circ x=x$. We conclude that $I$ has an idempotent element.

2. Let $v \in J$ and $p \in I$. Observe that $I \circ v \subseteq I$ and it is also a left ideal. Then, by minimality of $I$ we conclude that $I \circ v=I$. This implies that there is an element $q \in I$ such that $q \circ v=p$, then $p \circ v=(q \circ v) \circ v=q \circ v=p$.

3. We will see that $(v \circ I, \circ, v)$ is a group. We already know that $\circ$ is an associative operation, so we need to show that $v$ is an identity and the existence of inverses. Let $p \in v \circ I$, then there is some element $q \in I$, such that $p=v \circ q$. By (2), $v$ is right identity, and $v \circ p=(v \circ v) \circ q=v \circ q=p$. 
Now, let $q \in v \circ I$, we want to find some element $p \in v \circ I$ such that $p \circ q=v$. Observe that $I \circ q \subseteq I$ and it is also a left ideal, then $I \circ q=I$. Then, there is an element $p^{\prime} \in I$ such that $p^{\prime} \circ q=v$. Define $p=v \circ p^{\prime} \in v \circ I$, then we have that $p \circ q=\left(v \circ p^{\prime}\right) \circ q=v \circ\left(p^{\prime} \circ q\right)=v \circ v=v$, as required.

Remark 5.2.10. Notice that the first statement requires only that each function $S_{x}$ is continuos and closed.

Lemma 5.2.11. Let $(G, X, \pi)$ be a $G$-flow and $E(X)$ its enveloping semigroup. Let $I, K$ be left minimal ideals in $E(X)$. Then:

1. Given any idempotent $u \in I$ there exists a unique idempotent $v \in K$ such that $v \circ u=u$ and $u \circ v=v$.

2. The $G$-flows $(G, I)$ and $(G, K)$ are isomorphic.

3. $(u \circ I, \circ, u)$ is isomorphic to $(v \circ K, \circ, v)$.

Proof. 1. Let $u \in I$ be an idempotent element. Notice that $K \circ u \subseteq E(X) \circ u \subseteq I$, because $I$ is a left ideal. Moreover, $K \circ u$ is also a left ideal, since $E(X) \circ(K \circ u)=$ $(E(X) \circ K) \circ u \subseteq K \circ u$, because $K$ is also a left ideal. Since $I$ is a minimal left ideal,this implies that $K \circ u=I$. Let $L=\{p \in K \mid p \circ u=u\}$, which is a non-empty set because $K \circ u=I$. Moreover, $L$ is a closed subset of $E(X)$, since $L=S_{u}^{-1}(u) \cap K$. ( Remember that $S_{u}$ is continuous and $\{u\}$ is a closed set, because $E(X)$ is a Hausdorff space. Additionally, $K$ is closed because it is a minimal left ideal [see 5.2.7]). Additionally, $L$ is a subsemigroup of $K$, because it is closed under composition: let $p_{1}, p_{2} \in L$, then we have that

$$
\left(p_{1} \circ p_{2}\right) \circ u=p_{1} \circ\left(p_{2} \circ u\right)=p_{1} \circ u=u \text {. }
$$


Since $L$ is closed the restriction of each function $S_{x}$ to $L$ stills continuous and closed, therefore there is an idempotent element $v \in L$, this means that $v \circ v=v$ and $v \circ u=u$. (see 5.2.10)

Similarly, we can show that there is an element $w \in I$ such that $w \circ w=w$ and $w \circ v=v$ and by the previous Lemma (2) we have that $u \circ w=u$ and $w \circ u=w$. Thus

$$
w=w \circ u=w \circ(v \circ u)=(w \circ v) \circ u=v \circ u=u .
$$

So we conclude that, $v \circ u=u$ and $u \circ v=v$ and this implies the existence. Now we proceed in order to show the uniqueness, let $v_{1} \in K$ such that $v_{1} \circ v_{1}=v_{1}$, $u \circ v_{1}=v_{1}$ and $v_{1} \circ u=u$. Thus we have:

$$
v_{1}=v_{1} \circ v=v_{1} \circ(u \circ v)=\left(v_{1} \circ u\right) \circ v=u \circ v=v
$$

as required.

2. Let $u, v$ as in (1). Then, for any element $p \in I$, we have that $S_{u} \circ S_{v}(p)=(p \circ v) \circ u=$ $p \circ u=p$. Similarly, for any element $x \in K$ we have that $S_{v} \circ S_{u}(x)=x$, which shows that $S_{v}$ is an isomorphism from $(G, I)$ to $(G, K)$ which inverse is $S_{u}$.

3. Consider $S_{v}:(u \circ I) \rightarrow(v \circ K)$, notice that $S_{v}(u \circ p)=(u \circ p) \circ v \in K$, since $K$ is a left ideal. Moreover, $S_{v}(u \circ p) \in v \circ K$, since $u=v \circ u$ we have that $S_{v}(u \circ p)=v(u \circ p \circ v) \in v \circ K$.

We will show that $S_{v}$ induces an isomorphism between $u \circ I$ and $v \circ K$.

In first place observe that

$$
\phi(u \circ u)=\phi(u)=u \circ v=v .
$$


On the other hand,

$$
\begin{gathered}
S_{v}\left(\left(u \circ p_{1}\right) \circ\left(u \circ p_{2}\right)\right)=\left(u \circ p_{1}\right) \circ\left(u \circ p_{2}\right) \circ v= \\
\left(u \circ p_{1} \circ v\right) \circ\left(u \circ p_{2} \circ v\right)=S_{v}\left(u \circ p_{1}\right) \circ S_{v}\left(u \circ p_{2}\right) .
\end{gathered}
$$

Additionally, notice that $S_{v}$ and $S_{u}$ are both bijective by the observation that we have done in the previous item.

\subsection{Topological dynamics from a model theoretic ap- proach}

Newelski developed a model theoretic approach of the topological dynamics. Indeed, if we have a definable group $G$ then we can consider its action on $S_{G}(\mathcal{M})$, which is compact and Hausdorff. As we have seen, we can take the enveloping semigroup of this action $E\left(S_{G}(\mathcal{M})\right)$, and take a minimal left ideal $I$. We already know that for an idempotent element $u \in I$ we have that $(u \circ I, \circ, u)$ is a group. Thus the main question is if this group is isomorphic to $G / G^{00}$, and this is known as the Ellis semigroup conjecture. We give an affirmative answer for the definable bounded groups in Presburger Arithmetic, using a result proved by A. Pillay presented in [9].

Remark 5.3.1. $\quad$ 1. Let $\mathcal{A}$ be a model, we will indicate as $S(\mathcal{A})$ the set of the complete types with parameters in $\mathcal{A}$. Also, $G(\mathcal{A})$ will denote the set of realizations of $G$ in $\mathcal{A}$.

2. Let $G$ be a definable group and let $\mathcal{A}$ be a small model such that $G$ is definable over $\mathcal{A}$. We will denote as $S_{G}(\mathcal{A})=\{p(\bar{x}) \in S(\mathcal{A}) \mid(\bar{x} \in G) \in p(\bar{x})\}$. 
3. Let $\mathcal{M}_{0}$ be a small elementary submodel of $\mathcal{M}$, we will denote as

$$
S_{G}^{\mathcal{M}_{0}}(\mathcal{M})=\left\{p(\bar{x}) \in S_{G}(\mathcal{M}) \mid p(\bar{x}) \text { is finitely satisfiable in } \mathcal{M}_{0}\right\}
$$

Lemma 5.3.2. Let $\mathcal{A}$ be a model, then $\left(G(\mathcal{A}), S_{G}(\mathcal{A}), \pi\right)$ is a $G$-flow, where the action $\pi: G(\mathcal{A}) \times S_{G}(\mathcal{A}) \rightarrow S_{G}(\mathcal{A})$ is defined as $\pi(g \cdot p)=\left\{\phi\left(g^{-1} \cdot x\right) \mid \phi(x) \in p\right\}$.

Proof. - Step 1: $S_{G}(\mathcal{A})$ is a compact Hausdorff space.

The topology induced over $S_{G}(\mathcal{A})$ is the logic topology, whose basic open sets are $[\phi(x)]=\left\{p \in S_{G}(\mathcal{A}) \mid \phi(x) \in p\right\}$, where $\phi(x)$ is an $\mathcal{L}(\mathcal{A})$ - formula.

- $S_{G}(\mathcal{A})$ is a Haudorff space: assume that $p, q \in S_{G}(\mathcal{A})$ are different. Then there is an $\mathcal{L}(\mathcal{A})$ formula $\phi(x)$ such that $\phi(x) \in p$ and $\neg(\phi(x)) \in q$, therefore $p \in[\phi(x)]$ and $q \in[\neg(\phi(x))]$ and $[\phi(x)],[\neg(\phi(x))]$ are clearly disjoint.

- $S_{G}(\mathcal{A})$ is a compact it is enough to show that a family $\mathcal{F}$ of basic closed sets with the finite intersection property has a non empty intersection.

Let $\mathcal{F}=\left\{\left[\phi_{\alpha}(x)\right] \mid \alpha \in \beta\right\}$ be a family with the finite intersection property. Then, by compactness $\bigwedge_{\alpha \in \beta} \phi_{\alpha}(x)$ is consistent, therefore there is a type $p \in \bigcap_{\alpha \in \beta}[\phi(x)]$. We conclude that $S_{G}(\mathcal{A})$ is compact.

- Step 2: For each $g \in G(\mathcal{A}), \pi_{g}: S_{G}(\mathcal{A}) \rightarrow S_{G}(\mathcal{A})$ is an homeomorphism

$-\pi_{g}$ is bijective injective: Let $p, q \in S_{G}(\mathcal{A})$ be such that $p \neq q$. Then, there is an $\mathcal{L}(\mathcal{A})$ formula such that $\phi(x) \in p$ and $\neg(\phi(x)) \in q$, then $\phi\left(g^{-1} \cdot x\right) \in \pi_{g}(p)$ and $\neg\left(\phi\left(g^{-1} \cdot x\right)\right) \in \pi_{g}(q)$, which implies that $\pi_{g}(p) \neq \pi_{g}(q)$.

surjective: Let $p \in S_{G}(\mathcal{A})$. Define $q=g^{-1} \cdot p=\{\phi(g \cdot x) \mid \phi(x) \in p\}$, and observe that $\pi_{g}\left(g^{-1} \cdot p\right)=p$, then $\pi_{g}$ is surjective.

- $\pi_{g}$ is continuous and open

It is enough to show that for every $\mathcal{L}(\mathcal{A})$ - formula $\pi_{g}^{-1}([\phi(x)])$ is open in $S_{G}(\mathcal{A})$. 
Observe that

$$
\begin{aligned}
\pi_{g}^{-1}([\phi(x)]) & =\left\{p \in S_{G}(\mathcal{A}) \mid g \cdot p \in[\phi(x)]\right\} \\
& =\left\{p \in S_{G}(\mathcal{A}) \mid \phi(x) \in g \cdot p\right\} \\
& =\left\{p \in S_{G}(\mathcal{A}) \mid \phi\left(g^{-1} \cdot x\right) \in p\right\}=\left[\phi\left(g^{-1} \cdot x\right)\right]
\end{aligned}
$$

Similarly, we can prove that $\pi_{g}$ is also open.

Lemma 5.3.3. $S_{G}^{\mathcal{M}_{0}}(\mathcal{M})$ is a closed subset of $S_{G}(\mathcal{M})$ :

Let $p \in \overline{S_{G}^{\mathcal{M}_{0}}(\mathcal{M})}$, and consider $\phi(x)$ an $\mathcal{L}(\mathcal{M})$ formula such that $p \in[\phi(x)]$. Then, there is some type $q \in S_{G}^{\mathcal{M}_{0}}(\mathcal{M})$, such that $q \in[\phi(x)]$, therefore $\phi(x) \in q$, which implies that $\phi\left(\mathcal{M}_{0}\right) \neq \emptyset$. We conclude that $p$ is finitely satisfiable over $\mathcal{M}_{0}$, then $S_{G}^{\mathcal{M}_{0}}(\mathcal{M})$ is closed.

Lemma 5.3.4. Let $G e n=\left\{p \in S_{G}(\mathcal{M}) \mid p\right.$ is generic $\}$. This set is a closed $G(\mathcal{M})$ invariant subset of $S_{G}(\mathcal{M})$.

Proof. - Gen is closed: let $p \in \overline{G e n}$ and consider a formula $\phi(x)$ such that $p \in[\phi(x)]$. Therefore, there is a type $q \in G e n \cap[\phi(x)]$, which implies that $\phi(x) \in q$, then $\phi(x)$ is generic. We conclude that $p$ is also generic.

- Gen is $G(\mathcal{M})$-invariant: let $g \in G(M)$ and $p \in G$. Take a formula $\phi(x) \in g \cdot p$ and observe that it is a translation of a formula in $p$. Since $p$ is generic, $\phi(x)$ should also be generic. Then $G e n$ is an invariant subset of $G(\mathcal{M})$.

Remark 5.3.5. Right now we have several structures: such as $\left(G\left(\mathcal{M}_{0}\right), S_{G}^{\mathcal{M}_{0}}(\mathcal{M})\right)$ and $\left(G\left(\mathcal{M}_{0}\right), E\left(S_{G}\left(\mathcal{M}_{0}\right)\right)\right)$ as $G\left(\mathcal{M}_{0}\right)$-flows. We also may consider $\left(E\left(S_{G}\left(\mathcal{M}_{0}\right)\right), \circ\right)$ as a semigroup. The claim of the following lemma is to show that all these operations coincide, 
since we are going to show that the first two structures are isomorphic. Additionally, we prove that the isomorphism between $S_{G}^{\mathcal{M}_{0}}$ and $E\left(S_{G}\left(\mathcal{M}_{0}\right)\right)$ allows us to induce an operation on $S_{G}^{\mathcal{M}_{0}}$ from the o of $E\left(S_{G}\left(\mathcal{M}_{0}\right)\right)$, and this implies that $S_{G}^{\mathcal{M}_{0}}$ can be viewed also as a semigroup. All these results permit to us to denote all the operations as - without any kind of ambiguity.

Lemma 5.3.6. Let $G$ be a definable group and $\mathcal{M}_{0}$ be a small elementary submodel of $\mathcal{M}$, where $G$ is definable. Then:

- there is a natural homeomorphism between the compact space $E\left(S_{G}\left(\mathcal{M}_{0}\right)\right)$ and $S_{G}^{\mathcal{M}_{0}}(\mathcal{M})$,

- under this homemorphism, the Ellis semigroup operation of $E\left(S_{G}\left(\mathcal{M}_{0}\right)\right)$ becomes the following operation:

$$
\begin{aligned}
\cdot: S_{G}^{\mathcal{M}_{0}}(\mathcal{M}) \times S_{G}^{\mathcal{M}_{0}}(\mathcal{M}) & \rightarrow S_{G}^{\mathcal{M}_{0}}(\mathcal{M}) \\
(p, q) & \rightarrow t p(a \cdot b / \mathcal{M})
\end{aligned}
$$

where, $b$ realize $q$ and a realize $p \mid(\mathcal{M}, b)$.

Proof.

Let $p \in S_{G}^{\mathcal{M}_{0}}(\mathcal{M})$ and let $a$ be a realization of $p$. Then, we can consider the function $\pi_{p}: S_{G}\left(\mathcal{M}_{0}\right) \rightarrow S_{G}\left(\mathcal{M}_{0}\right)$, defined as $\pi_{p}(q)=t p\left(a \cdot b / \mathcal{M}_{0}\right)$, where $b \in G(\mathcal{M})$ and it is a realization of $q$.

Claim 1: $\pi_{p}$ is well defined for any $p \in S_{G}^{\mathcal{M}_{0}}(\mathcal{M})$

Let $a, a_{1} \in G(\mathcal{M})$ be such that $a, a_{1} \vDash p$. Let $q \in S_{G}\left(\mathcal{M}_{0}\right)$ and let $b, b_{1} \in G(\mathcal{M})$ be realizations of $q$, then it is enough to show that $t p\left(a \cdot b / \mathcal{M}_{0}\right)=t p\left(a_{1} \cdot b_{1} / \mathcal{M}_{0}\right)$. In order to get a contradiction assume that these types are different, then there is a formula $\phi(x, m)$ such that $\phi(x, m) \in t p\left(a \cdot b / \mathcal{M}_{0}\right)$ and $\neg(\phi(x, m)) \in t p\left(a_{1} \cdot b_{1} / \mathcal{M}_{0}\right)$, then $\phi\left(x \cdot b^{-1}, m\right) \in$ $t p(a / \mathcal{M})=p$ and $\phi\left(x \cdot b_{1}^{-1}, m\right) \in t p\left(a_{1} / \mathcal{M}\right)=p$. However, $(b, m) \equiv_{\mathcal{M}_{0}}\left(b_{1}, m\right)$, then 
$p$ is not $\mathcal{M}_{0}$-invariant. ( Recall: any type finitely satisfiable over a small model $\mathcal{M}_{0}$ is $\mathcal{M}_{0}$-invariant).

Claim 2: $\pi_{p} \in \overline{\left\{\pi_{g} \mid g \in G\left(\mathcal{M}_{0}\right)\right\}}$.

Let $q_{1}, \ldots, q_{n} \in S_{G}\left(\mathcal{M}_{0}\right)$ and $b_{1}, \ldots, b_{n} \in G(\mathcal{M})$ their respective realizations. Let $\phi_{1}(x), \ldots, \phi_{n}(x)$ be $\mathcal{L}(\mathcal{M})$ - formulas such that for each $1 \leq i \leq n, \pi_{p}\left(q_{i}\right) \in\left[\phi_{i}(x)\right]$. Therefore, $\mathcal{M} \vDash \bigwedge_{i=1}^{n} \phi_{i}\left(a \cdot b_{i}\right)$, it means that $\bigwedge_{i=1}^{n} \phi_{i}\left(x \cdot b_{i}\right) \in p$. Since $p$ is finitely satisfiable over $\mathcal{M}_{0}$, then there is an element $g \in G\left(\mathcal{M}_{0}\right)$ such that $\mathcal{M} \vDash \bigwedge_{i=1}^{n} \phi_{i}\left(g \cdot b_{i}\right)$. So we deduce that for any $1 \leq i \leq n$ we have $\pi_{g}\left(q_{i}\right) \in\left[\phi_{i}(x)\right]$, then we conclude that $\pi_{p} \in \overline{\left\{\pi_{g} \mid g \in G\left(\mathcal{M}_{0}\right)\right\}}$.

Then, we can consider the function $\phi: S_{G}^{\mathcal{M}_{0}}(\mathcal{M}) \rightarrow E\left(S_{G}\left(\mathcal{M}_{0}\right)\right)$ defined as $\phi(p)=\pi_{p}$. On the other hand, it follows by compactness that any function $f \in E\left(S_{G}\left(\mathcal{M}_{0}\right)\right)$ has the form $\pi_{p}$ for some $p \in S_{G}^{\mathcal{M}_{0}}(\mathcal{M})$. (Notice that each point of the function can be viewed as the intersection of several formulas).

Moreover, for any $p \in S_{G}^{\mathcal{M}_{0}}(\mathcal{M})$, it is uniquely determined by $\pi_{p}$, so $\phi$ is bijective.

Claim 3: $\phi$ is an homeomorphism.

Let $\psi_{1}(x), \ldots, \psi_{n}(x)$ be $\mathcal{M}_{0}$-formulas and take $q_{1}, \ldots, q_{n} \in S_{G}\left(\mathcal{M}_{0}\right)$. Let $b_{1}, \ldots, b_{n} \in$ $G(\mathcal{M})$ be realizations of $q_{1}, \ldots, q_{n}$ respectively. Let

$$
\mathcal{O}=\left\{f \in E\left(S_{G}^{\mathcal{M}_{0}}(\mathcal{M})\right) \mid \forall 1 \leq i \leq n, f\left(q_{i}\right) \in\left[\psi_{i}(x)\right]\right\}
$$


which is a basic open set of $E\left(S_{G}^{\mathcal{M}_{0}}(\mathcal{M})\right)$. Notice that:

$$
\begin{aligned}
\phi^{-1}(\mathcal{O}) & =\left\{p \in S_{G}^{\mathcal{M}_{0}}(\mathcal{M}) \mid \pi_{p} \in \mathcal{O}\right\} \\
& =\left\{p \in S_{G}^{\mathcal{M}_{0}}(\mathcal{M}) \mid \forall 1 \leq i \leq n, \pi_{p}\left(q_{i}\right) \in\left[\psi_{i}\right]\right\} \\
& =\left\{p \in S_{G}^{\mathcal{M}_{0}}(\mathcal{M}) \mid \bigwedge_{i=1}^{n} \psi_{i}\left(x \cdot b_{i}^{-1}\right) \in p\right\} \\
& =\left\{p \in S_{G}^{\mathcal{M}_{0}}(\mathcal{M}) \mid \forall 1 \leq i \leq n, p \in\left[\psi_{i}\left(x \cdot b_{i}^{-1}\right)\right]\right\}
\end{aligned}
$$

Then $\phi$ is continuous, this implies that it is also closed since it is continuous function between two compact Hausdorff spaces.

Claim 4: $\phi$ is a $G\left(\mathcal{M}_{0}\right)$ - flow homomorphism.

We already know that $\phi: S_{G}^{\mathcal{M}_{0}}(\mathcal{M}) \rightarrow E\left(S_{G}\left(\mathcal{M}_{0}\right)\right)$ is an homeomorphism. Then, we just need to prove that for every element $g \in G\left(\mathcal{M}_{0}\right)$ and $p \in S_{\mathcal{M}_{0}}^{G}(\mathcal{M})$ we have $\phi(g \cdot p)=\pi_{g} \cdot \phi(p)$. By definition of $\phi, \phi(g \cdot p)=\pi_{g \cdot p}$ so it is enough to show that for any type $q \in S_{G}\left(\mathcal{M}_{0}\right)$ we have that $\pi_{g \cdot p}(q)=\pi_{g} \circ \pi_{p}(q)$. Fix a realization $b \vDash q$, and let $a$ be a realization of $\left.p\right|_{(\mathcal{M}, b)}$. Then, $g \cdot a \vDash g \cdot p$, so $\pi_{g \cdot p}(q)=t p((g \cdot a) \cdot b / \mathcal{M})$, while $\pi_{g} \circ \pi_{p}(q)=\pi_{g}(t p(a \cdot b / \mathcal{M}))=t p(g \cdot(a \cdot b) / \mathcal{M})$. We conclude that $\pi_{g \cdot p}$ and $\pi_{g} \circ \pi_{p}$ coincide in any point $q$, so they are actually the same function.

Since $\phi$ is a $G\left(\mathcal{M}_{0}\right)$-isomorphism, then we conclude that $\left(G\left(\mathcal{M}_{0}, E\left(S_{G}\left(\mathcal{M}_{0}\right)\right)\right.\right.$ and $\left(G\left(\mathcal{M}_{0}\right), S_{G}^{\mathcal{M}_{0}}(\mathcal{M})\right)$ are isomorphic as $G$ flows.

Claim 5: $\left(E\left(S_{G}\left(\mathcal{M}_{0}\right), \circ\right)\right.$ and $\left(S_{G}^{\mathcal{M}_{0}}(\mathcal{M}), \cdot\right)$ coincide also as semigroups.

On the other hand, let $p, q \in S_{G}^{\mathcal{M}_{0}}(\mathcal{M})$ fix a realization $b \vDash q$ and $\left.a \vDash p\right|_{\mathcal{M}, b}$. Define the operation over $S_{G}^{\mathcal{M}_{0}}(\mathcal{M})$ as: $p \cdot q=t p(a \cdot b / \mathcal{M})$. Notice that this operation comes from the previous isomorphism. Fix any type $r \in S_{G}\left(\mathcal{M}_{0}\right)$ and let $c \vDash r$, thus $\phi(p \cdot q)(r)=$ 
$\pi_{p \cdot q}(r)=t p((a \cdot b) \cdot c / \mathcal{M})$. On the other hand, we have

$$
\pi_{p} \circ \pi_{q}(r)=\pi_{p}(t p(b \cdot c / \mathcal{M}))=t p(a \cdot(b \cdot c) / \mathcal{M})
$$

Therefore, $\phi(p \cdot q)=\phi(p) \circ \phi(q)$.

Remark 5.3.7. Remember that given a certain model $\mathcal{M}$, we can identify $G(\mathcal{M})$ inside of $S_{G}(\mathcal{M})$, considering the function:

$$
\begin{aligned}
f: G(\mathcal{M}) & \rightarrow S_{G}(\mathcal{M}) \\
g & \rightarrow t p(g / \mathcal{M})
\end{aligned}
$$

This function is injective and notice that it induces an isomorphism of groups between $(G(\mathcal{M}), \cdot)$ and $\left(\operatorname{Im}(f), \cdot^{\prime}\right)$ (the second operation is the one mentioned above). Indeed, $f(g \cdot h)=t p(g \cdot h / \mathcal{M})=f(g) \cdot f(h)$, because $g \vDash t p(g / \mathcal{M})$ and $h \vDash t p(h / \mathcal{M})$. Moreover, the function $f$ induces also an isomorphism between $(G(\mathcal{M}), \cdot)$ and $\left(\operatorname{Im}(f), \cdot^{\prime}\right)$ as topological groups. Notice that $f$ is continuos, because given an a $\mathcal{L}(\mathcal{M})$-formula $\phi(x)$, then $f^{-1}([\phi(x)])=\phi(\mathcal{M}) \cap G(\mathcal{M})$.

Additionally, $f$ is closed because given a closed set of $G(\mathcal{M})$ such as $C=\bigcap_{\beta<\alpha} \phi_{\beta}(\mathcal{M})$, we have that

$$
f(C)=\bigcap_{\beta<\alpha}\left[\phi_{\beta}(x)\right]
$$

which is also closed. Due this observation, in the following statements we will identify $G(\mathcal{M}), G^{00}(\mathcal{M})$ as subsets of $S_{G}(\mathcal{M})$, and we will consider their quotient group $G(\mathcal{M}) / G^{00}(\mathcal{M})$ ( which is isomorphic to the original quotient).

Theorem 5.3.8. Let $G$ be a definable bounded group. Consider the $G\left(\mathcal{M}_{0}\right)$-flow $\left(G\left(\mathcal{M}_{0}\right), S_{G}\left(\mathcal{M}_{0}\right)\right)$ and let $S=\left(E\left(S_{G}\left(\mathcal{M}_{0}\right)\right), \cdot\right)$ be its enveloping semigroup. Then for any minimal closed left ideal $I$ of $S$ and an idempotent element $u \in I$, the group $u \cdot I$ is isomorphic to $G / G^{00}$. 
Proof. Since $G$ has fsg, then we have that $G e n \neq \emptyset$. Additionally, we already know that $G e n$ is a closed and $G\left(\mathcal{M}_{0}\right)$-invariant set. By the lemma (2), we can conclude that $G e n \subseteq S_{G}^{\mathcal{M}_{0}}(\mathcal{M})$, therefore we have that $\left(G\left(\mathcal{M}_{0}\right), G e n\right)$ is a subflow of $\left(G\left(\mathcal{M}_{0}\right), S_{G}^{\mathcal{M}_{0}}(\mathcal{M})\right)$

Step 1: $\left(G\left(\mathcal{M}_{0}\right), G e n\right)$ is a minimal subflow of $\left(G\left(\mathcal{M}_{0}\right), S_{G}^{\mathcal{M}_{0}}(\mathcal{M})\right)$. Moreover, it is the unique minimal subflow of $\left(G\left(\mathcal{M}_{0}\right), S_{G}^{\mathcal{M}_{0}}(\mathcal{M})\right)$.

We just need to prove that it is minimal. Let $p \in G e n$, we will show that $\overline{G \cdot p}=G e n$. Let $q \in G e n$ and $\phi(\bar{x}, \bar{m})$ be a $\mathcal{L}(\mathcal{M})$. formula, such that $q \in[\phi(x, \bar{m})]$. Since $q$ is a generic type there are $g_{1}, \ldots, g_{k} \in \mathcal{M}_{0}$ elements such that $(x \in G) \leftrightarrow \bigcup_{i=1}^{k} g_{i} \cdot \phi(\bar{x}, \bar{m})$. Since $p$ is a complete type and $(x \in G) \in p$, then there is some $i \leq k$ such that $g_{i} \cdot \phi(\bar{x}, \bar{m}) \in p$, which implies that $\phi(\bar{x}, \bar{m}) \in g_{i}^{-1} \cdot p$. Therefore $G \cdot p \cap[\phi(\bar{x}, \bar{m})] \neq \emptyset$ and this is enough to say that $G e n=\overline{G \cdot p}$, thus $\left(G\left(\mathcal{M}_{0}, G e n\right)\right.$ is a minimal flow.

Now, we are going to prove that it is the unique minimal subflow, following a very similar argument as the one presented above. Let $I$ be another subflow and take an element $q \in I$. Fix a type $p \in G e n$, we will show that $q \in \overline{G \cdot p}$. So let $\phi(\bar{x}, \bar{m})$ be a $\mathcal{L}(\mathcal{M})$-formula, such that $\phi(\bar{x}, \bar{m}) \in q$. Following the same argument as before, we are able to find an element $g \in \mathcal{M}_{0}$ such that $g \cdot \phi(\bar{x}, \bar{m}) \in p$, which implies that $\phi(\bar{x}, \bar{m}) \in g^{-1} \cdot p$, thus $G\left(\mathcal{M}_{0}\right) \cap[\phi(\bar{x}, \bar{m})] \neq \emptyset$. So, we conclude that $q \in \overline{G \cdot p}=G e n$. Since $q$ is an arbitrary point of $I$, we can say that $I \subseteq G e n$ and by minimality of Gen we deduce that $G e n=I$.

Now, recall that $\left(G\left(\mathcal{M}_{0}\right), S_{G}^{\mathcal{M}_{0}}(\mathcal{M})\right)$ and $\left(G\left(\mathcal{M}_{0}\right), E\left(S_{G}\left(\mathcal{M}_{0}\right)\right)\right)$ are isomorphic as $G\left(\mathcal{M}_{0}\right)$-flows and $\left(S_{G}^{\mathcal{M}_{0}}(\mathcal{M}), \cdot\right)$ and $\left(E\left(S_{G}\left(\mathcal{M}_{0}\right)\right), \circ\right)$ also coincide as semigroups. Let's call $\phi: S_{G}^{\mathcal{M}_{0}}(\mathcal{M}) \rightarrow S_{G}\left(\mathcal{M}_{0}\right)$ the function which induces the desired isomorphism. We have that $\left(G\left(\mathcal{M}_{0}\right), \phi(G e n)\right)$ is also a subflow of $\left(G\left(\mathcal{M}_{0}\right), E\left(S_{G}\left(\mathcal{M}_{0}\right)\right)\right)$ (by Lemma 5.1.8). By Lemma 5.2.7, since $\phi(G e n)$ is a left minimal ideal of $\left(E\left(S_{G}\left(\mathcal{M}_{0}\right)\right)\right.$, o) (as a semigroup), then it has an idempotent element $p^{\prime} \in \phi(G e n)$. Since $\phi$ is bijective, then we 
can choose $p=\phi^{-1}\left(p^{\prime}\right) \in G e n$, which is an idempotent element of $S_{G}^{\mathcal{M}_{0}}(\mathcal{M})$ (because $\left.p \cdot p=\phi^{-1}\left(p^{\prime}\right) \cdot \phi^{-1}\left(p^{\prime}\right)=\phi^{-1}\left(p^{\prime} \circ p^{\prime}\right)=\phi^{-1}\left(p^{\prime}\right)=p\right)$. Additionally, since $p^{\prime} \circ \phi($ Gen $)$ is a group and $\phi$ is an isomorphism, then we have that $(p \cdot G e n, \cdot p)$ is also a group, whose identity is $p$.

Step 2: $p \cdot$ Gen meets every coset of $G^{00}$ in exactly one point.

Observe that for any element $q \in(p \cdot G e n) \cap G^{00}$, since $G^{00}$ is the stabilizer of $p$ (see Lemma 5.3) and $p$ is te identity of $(p \cdot G e n, \cdot)$, we have $q=q \cdot p=p$. This implies that for any element $q \in(p \cdot G e n) \cap G^{00}$, should be equal to $p$.Now, assume that there are elements $q, r \in p \cdot G e n$ in the same coset of $G^{00}$. It means that $q \cdot G^{00}=r \cdot G^{00}$, thus $r^{-1} \cdot q \in(p \cdot G e n) \cap G^{00}$. Thus we have $r^{-1} \cdot q=p$, which implies that $q=r \cdot p=r$.

We conclude that $p \cdot$ Gen meets every coset of $G^{00}$ in exactly one point.

Step 3: $p \cdot$ Gen is isomorphic to $G / G^{00}$

Consider the function $f: p \circ G e n \rightarrow G / G^{00}$, which sends any type $q$ to the unique coset containing $q$. (Notice that this has sense since considering a saturated model $\mathcal{M}^{\prime}$ over $\mathcal{M}$ we know that $G\left(\mathcal{M}^{\prime}\right) / G^{00}\left(\mathcal{M}^{\prime}\right) \simeq G(\mathcal{M}) / G^{00}(\mathcal{M})$.) By the step 2 we know that this map is injective and it is also surjective because $p \in G^{00}$, so we can reach each coset just translating this type. Additionally, this map induces also a topological homeomorphism between $p \cdot$ Gen (with the quotient topology) and $G / G^{00}$ with the logic topology. In fact, we know that the topology on $G / G^{00}$ is precisely the one given by the map $g: S_{G}(\mathcal{M}) \rightarrow G / G^{00}$. Therefore, considering $\hat{f}: G e n \rightarrow G / G^{00}$, which is the function that sends a type $q$ to the coset that contains $q$, is also continuous because it is 
the restriction of $g$ to a closed set. Also, notice that $f=\hat{f} \circ \pi$, where

$$
\begin{aligned}
\pi: \text { Gen } & \rightarrow p \cdot \text { Gen } \\
q & \rightarrow p \cdot q
\end{aligned}
$$

And, by the universal property of the quotient space, since $\hat{f}$ is continuos then $f$ should be also continuos. Moreover, notice that $\pi$ is a continuos function (by definition of the quotient topology), so given a closed set in $p \cdot G e n$, we have that $\pi^{-1}(C)$ should be closed in Gen. Therefore, $\hat{f}\left(\pi^{-1}(C)\right)$ should be a closed set of $G / G^{00}$. Considering that $f \circ \pi=\hat{f}$, then we can conclude that $f(C)=\hat{f}\left(\pi^{-1}(C)\right)$, so $f$ should be also closed.

Corollary 5.3.9. For any definable bounded group in Presburger Arithmetic, the Ellis group conjecture (conjectured by Newelski) holds. 


\section{Bibliography}

[1] Joseph Auslander. Minimal Flows and their Extensions. Mathematics studies, 153, 1991.

[2] Oleg Belegradek, Ya' acov Peterzil and Frank Wagner. Quasi-O-Minimal Structures. J. Symbolic Logic,Volume 65, No. 3, 1115-1132. 2000.

[3] Raf Cluckers. Presburger sets and p-minimal fields. J. Symbolic Logic, Volume 68, Issue 1, 153-162. 2003.

[4] Alfred Dolich. Forking and independence in O-minimal theories. J. Symbolic Logic, Volume 69, No.1, 215-240. 2004.

[5] Clifton Ealy, Krzysztof Krupinski and Anand Pillay. Superrosy dependent groups having finitely satisfiable generics. Annals of Pure and Applied Logic, Volume 151, Issue 1, 1-21. 2008.

[6] Robert Ellis. Lectures on Topological Dynamics. W.A. Benjamin Inc, New York, 1969.

[7] Ehud Hrushovski, Yaacov Peterzil, and Anand Pillay. Groups, measures and the NIP. J. Amer. Math. Soc. 21, 563-596. 2008.

[8] Yaacov Peterzil and Anand Pillay. Generic sets in definably compact groups. Manuscripta Mathematicae, (to appear).

[9] Anand Pillay. Topological Dynamics and definable groups. J. Symbolic Logic, Volume 78, Issue 2, 657-666. 2013. 
[10] Pierre Simon. A Guide to NIP theories. http://www normalesup.org/ simon/NIP_ guide.pdf. 2015 .

[11] Stan Wagon. The Banach-Tarski Paradox. Cambridge University Press, New York. 1993.

[12] David Marker. Model theory: an introduction. Springer, New York. 2002.

[13] Pantelis Eleftheriou and Sergei Starchenko. Groups Definable in Ordered Vector Spaces over Ordered Division Rings. J. Symbolic Logic, Volume 72, No. 4, 1108-1140. 2007.

[14] Alessandro Berarducci, Margarita Otero, Yaacov Peterzil and Anand Pillay. A descending chain condition for groups definable in o -minimal structures. Annals of Pure and Applied Logic, Volume 134, Issue 2-3, 303-313. 2005.

[15] Katrin Tent and Martin Ziegler. A course in model theory. Lecture Notes in Logic, Cambridge University Press. 2012. 